\title{
Early Stem Cell Aging in the Mature Brain
}

Albina Ibrayeva1,2,3, Maxwell Bay 1,2,4,11, Elbert Pu'1,2,11, David Jörg ${ }^{8,9}$, Lei Peng ${ }^{1,2,4}$, Heechul Jun ${ }^{10}$, Naibo Zhang ${ }^{1,2}$, Daniel Aaron ${ }^{1,2}$, Congrui Lin ${ }^{1,2}$, Galen Resler ${ }^{1,2}$, Mi-Hyeon Jang $^{10}$, Benjamin D. Simons ${ }^{8,9}$ and Michael A. Bonaguidi ${ }^{1,2,3,4,5,6,7,12 \#}$

${ }^{1}$ Eli and Edythe Broad Center for Regenerative Medicine \& Stem Cell Research at USC, ${ }^{2}$ Department of Stem Cell Biology and Regenerative Medicine, ${ }^{3}$ USC Davis School - Buck Institute Graduate Program in the Biology of Aging, ${ }^{4}$ Neuroscience Graduate Program, W. M. Keck School of Medicine, ${ }^{5}$ Zilkha Neurogenetic Institute, ${ }^{6}$ Department of Biomedical Engineering, ${ }^{7}$ Davis School of Gerontology, University of Southern California, Los Angeles, CA 90033, USA;

${ }^{8}$ Cavendish Laboratory, Department of Physics, ${ }^{9}$ Gurdon Institute, University of Cambridge, CB3 OHE, UK;

${ }^{10}$ Department of Neurological Surgery, Mayo Clinic College of Medicine, Rochester, MN 55905, USA;

${ }^{11}$ These authors contributed equally

${ }^{12}$ Lead Contact

\#Correspondence should be addressed to:

Michael Bonaguidi, Ph.D.

Broad CIRM Center, USC Keck School of Medicine, 1425 San Pablo Street, BCC 412, Los Angeles, CA 90033, USA

Tel: 323-865-1276

E-mail: mbonagui@usc.edu 


\section{SUMMARY}

Stem cell dysfunction drives many age-related disorders. Identifying mechanisms that initiate stem cell dysfunction represent early targets to enhance tissue resiliency throughout life. Here, we pinpoint multiple factors that compromise neural stem cell (NSC) behavior in the adult hippocampus. We find that NSCs exhibit asynchronous depletion by identifying short-term (ST-NSC) and intermediate-term NSCs (IT-NSCs). ST-NSC divide rapidly to generate neurons and deplete in the young brain. Meanwhile, multipotent ITNSCs are maintained for months, but are pushed out of homeostasis by lengthening quiescence. Single cell transcriptome analysis of deep NSC quiescence revealed several hallmarks of cellular aging in the mature brain, including changes in tyrosine-protein kinases Abl1 and Abl2. Treatment with the Abl-inhibitor Imatinib was sufficient to overcome deep quiescence and restore NSC proliferation in the middle-aged brain. Further examination of mature NSC with old epidermal, hematopoietic and muscle stem cell transcriptomes identified consensus changes in stem cell aging. Our study elucidates multiple origins of adult neurogenesis decline and reveals that hippocampal NSCs are particularly vulnerable to a shared stem cell aging signature.

Key words: stem cell, aging, adult neurogenesis, clonal analysis, quiescence, proliferation, fate change, single cell RNA-seq, bioinformatics, Abl, Imatinib, intervention, hippocampus. 


\section{INTRODUCTION}

Aging is the progressive loss of physiological function due to accumulating cellular damage (Kennedy et al., 2014; López-Otín et al., 2013). This process occurs at a gradual and asynchronous rate where specific cells and then organ systems lose homeostasis. Aging has been historically investigated in chronological terms, with the majority of studies comparing young and old organisms. More recently, approaches to prevent aging at younger ages have become more common (Belsky et al., 2015; Gillman, 2005). Mechanisms that initiate aging are thought to represent early targets for promoting tissue resiliency throughout life. However, progress in slowing declining tissue function has been hampered by an inability to determine when and which cells begin to exhibit cellular aging (López-Otín et al., 2013).

Stem cells represent a key pillar of aging (Kennedy et al., 2014). Stem cells are present in many adult tissues and are responsible for tissue generation throughout life. In order to do so, they must balance new cell production with stem cell maintenance. However, this equilibrium is progressively lost during chronological aging in several tissues including the brain, blood, muscle and skin (Clevers, 2015; Goodell and Rando, 2015; Liu et al., 2013; Ziebell et al., 2018). Loss of stem cell function contributes to degeneration in tissue integrity and a reduced capacity for regeneration upon injury (Sahin and DePinho, 2010). Several lines of evidence indicate that stem cells exhibit cellular aging. Stem cells from older organisms display somatic mutations, epigenetic erosion, impaired cellular metabolism, oxidative stress and proteostasis damage (Berman et al., 2017; Goodell and Rando, 2015; Limke et al., 2002; López-Otín et al., 2013; Mattson et al., 1999; Southall et al., 2013). Yet, despite the implications that stem cells can act as integration sites for many of the mechanisms that drive aging, no common stem cell aging signature has been identified (Keyes and Fuchs, 2018).

Neural stem cells represent a cell type that could be particularly vulnerable to cellular aging. Adult neurogenesis persists throughout life in the subgranular zone (SGZ) in the dentate gyrus of the hippocampus and in the subventricular zone (SVZ) along the lateral ventricles (Kirschen et al., 2019; Kuhn et al., 2018; Obernier and Alvarez-Buylla, 2019; Toda and Gage, 2018). In the adult hippocampus, neural stem cells (NSCs) give rise to new dentate granule neurons and astrocytes through a sequential process of cell 
cycle entry and generation of intermediate progenitor cells (Bonaguidi et al., 2011; Bond et al., 2015). New neurons play critical roles in regulating neural plasticity as well as cognitive and affective behaviors, whereas deficits in adult hippocampal neurogenesis have been implicated in age-related brain disorders (Anacker and Hen, 2017; Choi et al., 2018; Peng and Bonaguidi, 2018). While hippocampal neurogenesis persists throughout adulthood, this process is highly compromised during chronological aging. Aged animals have significantly less neural stem cell numbers, stem cell proliferation, neuronal differentiation and newborn neuron survival compared to younger animals (Encinas et al., 2011; Heine et al., 2004; Kuhn et al., 1996; Ziebell et al., 2018). Further, NSCs in old animals exhibit hallmarks of cellular aging including deficits in proteostasis and receive high levels of inflammation (Kalamakis et al., 2019; Leeman et al., 2018). Yet, the hippocampus experiences a loss of neurogenesis early in the mature brain of rodents (Ben Abdallah et al., 2010; Morgenstern et al., 2008) and by middle-age in humans (Knoth et al., 2010; Moreno-Jiménez et al., 2019; Spalding et al., 2013). This decline is accompanied by epigenetic loss of DNA demethylation (Gontier et al., 2018), suggesting NSCs could become compromised early during chronological aging.

However, cellular origins driving early neurogenesis decline remain unclear. In one scenario, NSCs have been found to drop in number in the young hippocampus (Gontier et al., 2018; Lugert et al., 2012). This is supported by the presence of a finite NSC pool that lacks self-renewal capability and generates neurons (Encinas et al., 2011; Pilz et al., 2018). Consistently, forced accelerated neurogenesis (Ehm et al., 2010; Jones et al., 2015; Renault et al., 2009) and pathological conditions (Mu and Gage, 2011; Sierra et al., 2015) can result in premature NSC depletion. In another scenario, studies (Bonaguidi et al., 2011; Dranovsky et al., 2011; Licht et al., 2016) have shown that self-renewing NSCs are maintained and neurogenesis instead could decline during aging due to an increase in NSC quiescence (Hattiangady and Shetty, 2008; Ziebell et al., 2018). While NSCs as a population clearly undergo early age-related changes, when and how specific NSC subpopulation begin to exhibit dysfunction remain unclear. In this study, we used single cell approaches to investigate the cellular and molecular mechanisms that initially compromise adult neurogenesis. We determined that NSC subpopulations undergo asynchronous decline and exhibit cellular aging. We further identify a stem cell aging 
signature shared across multiple aged tissues. Finally, we show that targeting cellular aging in the middle-aged brain can overcome age-related neural stem cell dysfunction.

\section{RESULTS}

\section{Asynchronous NSC decline during aging}

We first investigated how adult NSC numbers change over time at the population level. The dentate gyrus (DG) of the hippocampus was harvested from wild-type mice and processed by immunofluorescence to reveal NSC behavior across chronological age. Nestin staining of radial-glial like neural stem cells indicated that NSC number decreases over time (Figure 1A-B). Consistent with prior observations, NSC loss is most pronounced transitioning from the young to mature and middle-aged brain (Gontier et al., 2018). We further processed neural stem cells for the cell cycle marker MCM2 to quantify age-related changes in radial-glia like NSC proliferation. Similar to findings in older mice (Encinas et al., 2011; Hattiangady and Shetty, 2008; Ziebell et al., 2018), remaining NSCs become more quiescent early in the mature brain (Figure 1B-C, Figure S1A). Therefore, radialglia like NSC number declines over time and remaining NSCs do not divide as frequently at the population level.

Various observations of NSC behavior have been made in the adult hippocampus. Some NSCs are short-lived and divide rapidly to mainly produce granule neurons exhibiting "division-coupled differentiation" behavior (Encinas et al., 2011) or "developmental-like model" behavior (Pilz et al., 2018). Meanwhile, some studies (Bonaguidi et al., 2011; Dranovsky et al., 2011; Jang et al., 2013; Licht et al., 2016) have advanced a stem cell maintenance model where NSCs are longer lived, slowly cycle in and out of quiescence, can generate neurons, astrocyte and self-renew. We reasoned that cellular heterogeneity within the radial glial-like NSC pool masks the ability to precisely uncover which mechanisms contribute to NSC dysfunction over time (Bonaguidi et al., 2012, 2016). We therefore performed in vivo single cell clonal analysis in NestinCreER $R^{T 2}\left(\right.$ Nestin $\left.^{\#}\right)$ and Ascl1-CreER ${ }^{T 2}\left(\right.$ Ascl1 $\left.^{\#}\right)$ mice according to our prior approaches (Bonaguidi et al., 2011). We found that clonally labelled Ascl1\#-NSCs represent a shortterm (ST-NSCs) neuronal fate-biased population, consistent with a developmental-like program (Pilz et al., 2018) (Figure 1D-F, S2B). Moreover, these ST-NSCs lack any 
detectable homeostasis due to a lack of symmetric self-renewing divisions (Figure 1G-J). Meanwhile, individual NSCs marked by Nestin" are slow cycling, generate neurons and astrocytes, and self-renew (Figure 1D-F, S2A). Importantly, these multipotent Nestin\#_ NSCs are homeostatic in the young brain for a few months - balancing their self-renewal and differentiation - then transition out of homeostasis at around 4.5-months-old mice (Figure 1G-J). As a result, these NSCs have twice the half-life of Ascl1\#-NSCs and increase their maintenance with age (Figure 1E-F). Thus, Nestin\#-NSCs represent intermediate-term NSCs (IT-NSCs) and serve as a platform to pinpoint mechanisms mediating the loss of stem cell homeostasis in the mature brain.

\section{Increased quiescence drives NSC loss of homeostasis}

To begin exploring why Nestin\#-NSCs lose homeostasis, we considered cellular mechanisms that would lead to declining NSC numbers. NSCs are homeostatic when their rate of production (expansion) balances their rate of differentiation (Figure 2A). We reasoned that NSCs either increase their differentiation or slow their expansion during their transition out of homeostasis. We first tested NSC clonal depletion from 2 to 6months-old mice to analyze the rate of NSC differentiation. As expected, Ascl1 ${ }^{\#-N S C s}$ displayed a rapid initial depletion that slowed with time. Meanwhile, Nestin\#-NSCs exhibited a constant and gradual loss of clones containing a single NSC, indicating that homeostasis decline does not occur due to accelerating NSC differentiation (Figure 2B). We then analyzed NSC-containing clones to identify age-related changes in the rate of NSC expansion. Ascl1"\#-NSCs displayed no appreciable expansion over time. On the other hand, Nestin\#-NSCs did expand in young mice according to values predicted to maintain homeostasis (Figure 2C). However, NSC expansion quickly began to diverge from levels needed to maintain homeostasis in approximately 4-month-old mice. Hence, Nestin ${ }^{\#-N S C s ~ l o s e ~ h o m e o s t a s i s ~ i n ~ t h e ~ m a t u r e ~ b r a i n ~ b e c a u s e ~ o f ~ s l o w i n g ~ N S C ~ p r o d u c t i o n ~}$ while Ascl1\#-NSCs do not exhibit homeostasis due to a lack of NSC production in the young brain (Figure 2A-C).

NSC expansion occurs in a two-step process: transition from quiescence to cell cycle and symmetric cell division. To determine which stem cell behavior drives the loss of NSC homeostasis, we performed cell cycle kinetics and cell fate analyses on Nestin\#- 
NSC clones. Two-months-old and six-months-old Nestin ${ }^{\#}$ mice were traced for 7 days, 1 month, 2 months and 4 months (Table S4). We then calculated the time to cell cycle entry and re-entry according to power-law decay principles (Figure 2D, (Pilz et al., 2018)). Both durations to the first and second division increased in Nestin\#-NSCs between 2- and 6month old mice (Figure 2E-F). These findings indicate that Nestin\#-NSCs display a substantial increase in quiescence in the young hippocampus and enter a "deeper quiescent" state in the mature brain (van Velthoven and Rando, 2019). To determine agerelated changes in cell fate choices, we followed the generation of each lineage type (neuronal, astroglial, NSCs) and lineage cell type (neuronal intermediate progenitor cells, immature neurons, mature neurons; astrocyte progenitor cells, astrocytes) within the Nestin\#-NSC clones. Fate choices were calculated for each cell division among the neuronal, astroglial and NSC lineages (Figure 2G-H). As previously reported (Bonaguidi et al., 2011), Nestin ${ }^{\#-N S C s ~ p r e d o m i n a t e l y ~ m a k e ~ n e u r o n a l ~ a n d ~ a s t r o g l i a l ~ a s y m m e t r i c ~ f a t e ~}$ choices in the young 2-month-old hippocampus. However, we found that symmetric cell divisions predominantly occur in the 6-month-old mature brain (Figure 2I, S2C-D). Together, these observations indicate that Nestin\#-NSCs prolong their quiescence with each division, which drives them from homeostasis in the mature hippocampus. Further, they then switch to symmetric cell fate choice after NSC homeostasis has been lost (Figure 2J).

\section{Quiescent NSC aging in the mature hippocampus}

NSCs are a rare cell population in the adult hippocampus that transition between quiescent and active states (Bonaguidi et al., 2011). While molecular signatures distinguishing quiescent from active NSCs have been well documented in the SVZ and hippocampus (Codega et al., 2015; Dulken et al., 2017; Llorens-Bobadilla et al., 2015; Shin et al., 2015), age-related changes within NSC quiescence have been more difficult to identify (Kalamakis et al., 2019; Leeman et al., 2018). Nestin\#-NSCs are homeostatic in the young hippocampus for a few months, then exit homeostasis at around 4.5-monthsold mice due to an increase in quiescence (Figures 1-2). To uncover molecular mechanisms of deepening NSC quiescence, we performed single cell RNA sequencing (scRNA-seq) (Figure 3A, S3A). NSCs and their immediate progeny were isolated using 2 
months-old and 4.5 months-old Nestin::CFP mice (Encinas et al., 2006), and immediately enriched using FACS (Figure S3B). Single-cell cDNA libraries were built using a miniaturized SMART-Seq4 protocol, barcoded, multiplexed and sequenced using pairedend NextSeq500 (Figure 3A, S3). Sequencing reads were aligned using STAR (Dobin et al., 2013) and expression estimates were computed from aligned reads with RSEM (Li and Dewey, 2011). After gene filtering, 14,750 expressed genes were detected across cells (average 3,600 genes per cell) - which allows for a deep exploration of NSC transcriptional dynamics within quiescence (Table S5-S6). We distinguished NSCs (Nestin, Aldoc, Vimentin, Apoe, Fabp7) from intermediate progenitor cells (IPCs: Eomes/Tbr2, Sox11, Stmn1) and identified NSCs in quiescent (negative for proliferative markers) or active states (PCNA, Mcm7, Cdk4, Cdk6) using well established markers (Shin et al., 2015) (Figure 3C, S4A-C). Importantly, both Ward minimum variance hierarchical clustering and principal component analysis (PCA) showed highly conserved cell-state-specific expression profiles between the two time points, indicating that global transcriptomic differences are greater between quiescent and active NSC states than within NSC states cells from 2-month-old and 4.5-month-old mice (Figure 3B, S4D-F).

Next, we probed for changes within quiescence between 2-month-old and 4.5month-old NSCs using RNA velocity. RNA velocity is a bioinformatic tool that takes separate observations of unspliced and spliced reads to predict the future gene expression state of individual cells on a timescale of hours and reveal lineage progression (La Manno et al., 2018). RNA vectors in our analysis indicated that most NSCs remain quiescent but when activated NSCs either differentiate or return to quiescence (Figure 3D-F). We did not detect age-related differences in activated NSCs or IPCs isolated from 2-month-old and 4.5-month-old mice. Interestingly, we found that NSCs in quiescence are more likely to stay in that state from 4.5-month-old mice compared to 2-month-old mice (Figure 3F), again indicating that NSCs begin to enter deeper quiescence as the hippocampus ages.

We then sought to identify transcriptomic changes associated with this NSC deep quiescence. 493 upregulated and 576 downregulated genes were differentially expressed (DE) in quiescent NSCs from 4.5-month-old mice compared to 2-month-old mice (Figure 3G, S4D-F, Table S8). We then used TopGo (Alexa et al., 2006) to represent DEs by their 
associated biological processes. Consistent with our lineage tracing experiments (Figure 2), gene ontology revealed changes in neurogenesis (Socs2, Wnt3, Epha4), a gain in gliogenesis (Ezh2, Disc1) and a loss of cell cycle (Wee1, Mcm6, Heca). In addition, we observed alterations in processes known to regulate adult NSC quiescence including semaphorin signaling (Plexna4, Plxnb3, Nrp2, Farp2), Ras signaling (Igf1, Rassf1, Abl1, Icmt) and Rho signaling (Spata13, Myo9b, Tiam2, Mcf2I, Scai) (Chavali et al., 2018; Jongbloets et al., 2017; Li et al., 2012) (Figure 3H-I, S5, Table S8). Strikingly, the remainder of detected terms are hallmarks of an intertwined process that drives cellular aging (Kennedy et al., 2014; López-Otín et al., 2013). These factors include epigenetic dysregulation from histone demethylation (Kdm1b, Kdm2a, Kdm4a, Kdm5d, Uty), downregulation of transcription (Sox11, Mycn, Creb1, Hif1an, Sertad2, Epcam), inflammation due to NIK/NF-kappaB signaling (Cd14, Malt1, Eif2ak2, Tirap, Myd88), and cellular stress from loss of DNA repair (Sesn2, Msh3, Msh6, Rfc3, Rfc5, Rad18), DNA recombination (Atad5, Hus1, Pms2, Lig3, Mcm9) and increased double-strand break repair (Chek1, BIm, Spidr, Fancb) (Figure 3H-J, S5, Table S8). Therefore, functional changes occurring in deep NSC quiescence are associated with early cellular aging in the mature hippocampus.

\section{Imatinib restores NSC proliferation in the middle-aged brain}

Strategies to delay and potentially even reverse the aging process have recently been developed (Mahmoudi et al., 2019). Since NSCs undergo early cellular aging in the mature brain (Figure 3H-I), we hypothesized that targeting this process could overcome age-related NSC dysfunction. Gene networks are powerful approaches with the ability to prioritize genes based upon their degree of connectedness to other genes and functions (Li and Horvath, 2007). We therefore probed for differentially expressed genes shared between 3 or more GO terms for target identification (Figure 3J). Our analysis identified Abl1, Abl2, Igf1, Lef1, Per2 and Nup62 as genes most connected to age-related NSC function - including changes in signal transduction, gene expression, cell cycle, DNA repair and neurogenesis (Figure $3 \mathrm{~J}$ ). We decided to focus on targeting Abl1 (ABL)/Abl2 (ARG) because of its many context-dependent signaling functions, but unknown role in NSC biology (Wang, 2014). We therefore inhibited Abl with Imatinib in vivo as a strategy 
to overcome NSC cellular aging. Imatinib or vehicle control was infused into the hippocampal fimbria of 10-month-old mice for 6 days (Figure 3K). Nestin staining of radialglial like NSCs with the cell cycle marker MCM2 revealed that Imatinib increases NSC proliferation in the middle-aged hippocampus (Figure 3L-M). Thus, targeting mechanisms associated with the initial loss of NSC homeostasis can overcome age-related NSC dysfunction later in life.

\section{Consensus signatures of stem cell aging}

Many aged tissues display functional decline due, in part, to stem cell dysfunction. Aging can compromise general stem cell fitness: their capacity to self-renew, activate from quiescence, and produce specialized cell types (Goodell and Rando, 2015). However, the relative contributions of intrinsic versus extrinsic factors on aging in each adult stem cell population are still unclear, and studies have to date not identified consensus aging signatures across multiple stem cell compartments (Keyes and Fuchs, 2018). Our study has identified that hippocampal NSCs exhibit early cellular aging (Figure 3). To extend this phenotype, we compared NSC transcriptomes from the mature hippocampus (merged quiescent and active states) with published advanced age hematopoietic - hSC (Sun et al., 2014), muscle - mSC (Liu et al., 2013) and epidermal eSC (Solanas et al., 2017) stem cell transcriptome datasets (Figure 4A, Table S9). Fisher's exact tests were run on respective DEs to determine the degree of ageassociated gene expression convergence between NSCs and other stem cell compartments. DEs driving cellular aging in NSCs compared against DEs from chronological aging in hSC, mSC, eSC showed statistically significant gene-level convergence in both expression directions (Figure 4B, Table S9). Further pairwise analysis of gene ontology between NSCs from the mature brain and old stem cells from other tissues indicated functional convergence of aging (Figure 4C). The degree of overlap between NSCs and mSCs, NSCs and eSCs was greater at the functional level than gene level (Figure 4B-C) suggesting that hippocampal aging is driven by an expression program that, although involves specific genes which are not widely shared with other stem cell compartments, nonetheless converges on the same biological processes of aging. 
We then investigated the presence of a consensus stem cell aging signature across 4 somatic stem cell compartments. Similar to prior reports (Keyes and Fuchs, 2018), only a few genes (1-10) were shared among 3 stem cells and none were convergent among all 4 stem cells (Figure S6A-B). Indeed, permuted convergence of DE genes decreased monotonically when examining pairs, triads and quads of stem cell niches (Figure S6A-B). This observation suggests that stem cell aging acts on a set of genes that are largely unique to any respective niche. Interestingly, ontology-level convergence decreased between pairs and triads, but increased again when observing the convergence between all four stem cells (Figure S6C-D, Table S10-S11). This finding suggests aging across stem cell niches recruit different but complementary genes which target common biological processes. In total, 19 gene ontology terms are differentially regulated in all 4 stem cells (Figure S6C-D, Table S10-S11). These terms revealed general downregulation of metabolic processes and upregulation of cell signaling (Figure 4D). We then performed a more focused enrichment analysis on the DEs which were component genes of metabolism to understand which specific aspects were perturbed with aging. This analysis revealed a downregulation of genes associated with metabolic changes in RNA homeostasis including RNA biosynthesis, DNA-templated transcription and RNA polymerase II driven transcription; and metabolic changes in proteostasis such as control of peptidyl-amino acid modifications and phosphorylation (Figure 4E, G). Similarly, we performed a focused enrichment analysis on upregulated component genes of cell signaling. We identified changes in WNT signaling, TGF $\beta$ signaling and cell death signaling pathways (Figure 4F, G, Table S12). Thus, our results suggest that stem cell aging is driven by a general loss of intrinsic factors and a gain in regulation of extrinsic factors.

Finally, we generated string networks to visualize the shared and unshared gene expression changes within biological processes which were associated with aging across all 4 stem cell data sets (Figure 4H-I). Different members of shared gene families were involved in aging across stem cells in every investigated ontology term among cell signaling, RNA homeostasis and proteostasis (Figures S7-9). Specifically, Eya transcription factor paralogs were downregulated in NSCs (Eya3), hSCs (Eya 4), mSCs and eSCs (Eya 2) (Figure 4H). In addition, regulators of RNA polymerase II were 
downregulated in all 4 stem cells, including members of Tet, Fox, Sox and Hox families (Figure 4I, Table S12). Thus, we have identified gene families that play complementary roles in stem cell aging across 4 tissues. Further, changes in old hematopoietic, muscle and epidermal stem cells are present early in neural stem cells from the mature hippocampus.

\section{DISCUSSION}

Determining when stem cells begin to exhibit age-related dysfunction is essential for identifying targets to slow tissue decline later in life. Here, we show that neural stem cells become compromised early in life within the mature hippocampus. Nestin\#-NSCs were found to temporarily balance self-renewal and differentiation but lose this homeostasis due to an increase in quiescence. NSCs in deep quiescence exhibited changes in cell fate and underwent cellular aging in the mature hippocampus. Importantly, targeting Abl components of this early cellular aging were able to overcome deep quiescence in middle-age mice. Finally, we defined the first consensus signature of stem cell aging among neural stem cells, epidermal stem cells, hematopoietic stem cells, and muscle stem cells. These observations suggest that NSCs in the hippocampus become compromised at an early age through conserved stem cell aging.

Neurogenesis is well known to decline with age. Old animals have significantly less NSC numbers, proliferation and neuronal differentiation compared to younger animals (Encinas et al., 2011; Heine et al., 2004; Kuhn et al., 1996; Ziebell et al., 2018). However, heterogeneity within the NSC pool has complicated the identification of the cellular origins initiating age-related neurogenesis decline (Bonaguidi et al., 2011; Dranovsky et al., 2011; Encinas et al., 2011; Pilz et al., 2018). Our single cell lineage tracing determined that asynchronous NSC dysfunction is a unifying principle of NSC aging. We found that Ascl1"-labeled radial cells are neuronal-biased ST-NSCs, whose number rapidly declines in the young hippocampus. These cells have a higher proliferation rate, primarily generate neurons, and are computationally consistent with "disposable" and "developmental-like" NSC behavior (Encinas et al., 2011; Pilz et al., 2018). Meanwhile, Nestin\#-labeled radial cells are multipotent IT-NSCs that divide and maintain their numbers while ST-NSCs differentiate. IT-NSCs produce neurons, astrocytes, stem cells, and are computationally 
consistent with "self-renewing" NSCs (Bonaguidi et al., 2011; Dranovsky et al., 2011). However, IT-NSCs increase their time in quiescence with each division until homeostasis is compromised and their numbers begin to decline in the mature brain. In addition, ITNSCs change their fate to symmetric divisions in 6 months-old mice while quiescence continues to increase into middle age. Therefore, hippocampal neurogenesis declines with age in a temporal pattern: (1) ST-NSC number declines in the young brain, (2) ITNSC quiescence increases in the young brain, (3) IT-NSC number declines in the mature brain, (4) IT-NSC fate changes in the mature brain, and (5) IT-NSC quiescence continues to deepen into the middle-aged brain. This sequence highlights the process of NSC aging and details multiple origins of neurogenesis decline during chronological aging (Kuhn et al., 1996, 2018).

While stem cells undergo cellular aging in many old tissues (Goodell and Rando, 2015; López-Otín et al., 2013), only recently have these mechanisms been explored in the brain. Quiescent neural stem cells in the old SVZ exhibit deficits in proteostasis and receive high levels of inflammation (Kalamakis et al., 2019; Leeman et al., 2018). However, transcriptome investigation between young and old SVZ NSCs has thus far not revealed substantial age-related differences. Instead, our clonal lineage in the hippocampus indicated that many changes in NSC function occur in the mature brain. We therefore utilized deeper sequencing to provide enhanced sensitivity (>1000 DEs) of agerelated transcriptomic changes in single NSCs. Remarkably, nearly all pillars of cellular aging (Kennedy et al., 2014) were found to change within NSC quiescence. Consistent with NSCs from the aged SVZ (Kalamakis et al., 2019; Leeman et al., 2018), NSCs from the mature hippocampus exhibited deficits in proteostasis and received inflammatory signals (NIK-NFkB). Additionally, we uncovered the new presence of age-related metabolic alterations, histone demethylase dysregulation, transcriptional downregulation, and DNA damage in NSCs. Our findings strongly implicate that NSCs exhibit early aging in the mature hippocampus and identified associated molecular mechanisms at the systems level.

To determine which pillars of cellular aging are expressed in stem cells more broadly, we sought convergence in transcriptomes from hippocampal NSC, epidermal stem cell, hematopoietic stem cell and muscle stem cells. Similar to prior studies (Keyes 
and Fuchs, 2018), we did not detect a single age-related gene shared among four stem cells. However, we determined common age-related functional deficits in metabolism, RNA homeostasis and proteostasis by gene ontology. Generating string networks to visualize gene-function relationships among 4 stem cells, we observed that gene families such as Eya, Tet, Fox, Sox, Hox and Zfps are expressed in complementary patterns across individual niches. Therefore, we found that specific gene families exhibit biological convergence and provide the first consensus signature of stem cell aging. In addition to the intrinsic factors that contribute a large extent to stem cell aging, we also identified changes in Wnt signaling, TGF $\beta$ signaling and apoptosis inhibition in 4 stem cell populations. While Wnt and TGF $\beta$ family signaling are well known modulators of stem cell activity during aging (Kalamakis et al., 2019; Oh et al., 2014), the role of extrinsic apoptotic signaling inhibition is less studied. Given that extrinsic manipulation of stem cell proliferative homeostasis can extend lifespan (Biteau et al., 2010) as can modulation of metabolism, RNA homeostasis and proteostasis (Chandel et al., 2016; Kim et al., 2018), we provide new targets to potentially coordinate stem cell activity across multiple tissues for treating age-related dysfunction.

Strategies to alter the aging process have recently been developed. Manipulation of blood factors, niche factors, or metabolism (diet and exercise) have demonstrated the capability to partially restore hippocampal neurogenesis and cognition (de Cabo et al., 2014; Gontier et al., 2018; Katsimpardi and Lledo, 2018; Mahmoudi et al., 2019). In addition, drugs such as rapamycin, metformin, and senoyltics (dasatinib, quercetin) have yielded promising results as potential anti-aging treatments (Leeman et al., 2018; Mahmoudi et al., 2019; Ogrodnik et al., 2019; Torres-Pérez et al., 2015). However, the vast majority of these studies have been performed in animals of advanced age (TorresPérez et al., 2015). Instead, age-related changes to physiology accumulate early in life, and interventions to reverse or delay age-related diseases are more effective earlier in disease etiology (Belsky et al., 2015; Gillman, 2005). We have taken a similar approach by identifying the initiation of NSC aging and functional decline in the mature brain. Importantly, targeting this process with Imatinib in the middle-aged brain was sufficient to overcome deep NSC quiescence. Since increased quiescence and resistance to enter cell-cycle occur in many old tissues that exhibit stem cell functional decline (Fan et al., 
2017; Goodell and Rando, 2015; Kalamakis et al., 2019), this approach may provide targets for early interventions in multiple tissues. Interestingly, unlike the next generation Abl inhibitor dasatinib, no senolytic activity has been observed by Imatinib treatment (Zhu et al., 2015). Therefore, understanding the key mechanisms that drive cellular aging will help to create new directions towards age-related regenerative medicine.

\section{ACKNOWLEDGMENTS}

We thank David Cobrinik, Sunhye Lee and members of the Bonaguidi, Simons and Jang laboratories for invaluable discussions and comments on the manuscript; Teresa Krieger, Dennisse Jimenez-Cyrus, Avinash lyer and Lee McMahon for technical support; CHLA FACS Core Facility, CHLA Center for Personalized Medicine Sequencing facility for technical assistance. This work was supported by grants to M.A.B. from the NIH NINDS (R00NS080913), Donald E. and Delia B. Baxter Foundation, Whittier Foundation and CHLA TSRI pilot program; M-H.J. was supported by a NIH NIA (RO1AG0585560); B.D.S. was supported by a Royal Society E P Abraham Professorship (RPIR1\180165) and Wellcome Trust (grant number 098357/Z/12/Z); A.I. was partially supported by the American Federation for Aging Research Scholarship for Research in Biology of Aging, and M.B. was partially supported by the USC Provost's Research Enhancement Fellowship.

\section{AUTHOR CONTRIBUTIONS}

Conceptualization: A.I., M.B. and M.A.B.; Data collection and methodology: A.I., E.P., L.P., N.Z., H.J., M.A.B., and C.L, G.R. with supervision; Data analysis and bioinformatics: A.I., M.B., D.J, L.P., D.A., B.S. and M.A.B.; Resources: M-H.J, B.S, M.A.B, Supervision: M-H.J, B.S, M.A.B.; Writing, Review and Editing: A.I., M.B. and M.A.B.; Project coordination: M.A.B. All authors commented on the manuscript.

\section{CONFLICT OF INTEREST}

No conflicts of interest to declare 


\section{REFERENCES:}

Ahn, S., and Joyner, A.L. (2005). In vivo analysis of quiescent adult neural stem cells responding to Sonic hedgehog. Nature 437, 1-4.

Alexa, A., Rahnenfuhrer, J., and Lengauer, T. (2006). Improved scoring of functional groups from gene expression data by decorrelating GO graph structure. Bioinformatics 22, 1600-1607. Alexa A and Rahnenfuhrer J (2016). topGO: Enrichment Analysis for Gene Ontology. Anacker, C., and Hen, R. (2017). Adult hippocampal neurogenesis and cognitive flexibility linking memory and mood. Nat. Rev. Neurosci. 18, 335-346.

Balordi, F., and Fishell, G. (2007). Mosaic Removal of Hedgehog Signaling in the Adult SVZ Reveals That the Residual Wild-Type Stem Cells Have a Limited Capacity for Self-Renewal. J. Neurosci. 27, 14248-14259.

Belsky, D.W., Caspi, A., Houts, R., Cohen, H.J., Corcoran, D.L., Danese, A., Harrington, H., Israel, S., Levine, M.E., Schaefer, J.D., et al. (2015). Quantification of biological aging in young adults. Proc. Natl. Acad. Sci. U. S. A. 112, E4104-10.

Berg, D., Postuma, R.B., Adler, C.H., Bloem, B.R., Chan, P., Dubois, B., Gasser, T., Goetz, C.G., Halliday, G., Joseph, L., et al. (2015). MDS research criteria for prodromal Parkinson's disease. Mov. Disord. 30, 1600-1611.

Berman, A.Y., Motechin, R.A., Wiesenfeld, M.Y., and Holz, M.K. (2017). The therapeutic potential of resveratrol: a review of clinical trials. NPJ Precis. Oncol. 1.

Biteau, B., Karpac, J., Supoyo, S., DeGennaro, M., Lehmann, R., and Jasper, H. (2010). Lifespan Extension by Preserving Proliferative Homeostasis in Drosophila. PLoS Genet. 6, e1001159.

Bolger, A.M., Lohse, M., and Usadel, B. (2014). Trimmomatic: a flexible trimmer for Illumina sequence data. Bioinformatics 30, 2114-2120.

Bonaguidi, M., Song, J., Ming, G.-L., and Song, H. (2012). A unifying hypothesis on mammalian neural stem cell properties in the adult hippocampus. Curr. Opin. Neurobiol. 22, 754-761.

Bonaguidi, M.A., Stadel, R.P., Berg, D.A., Sun, J., Ming, G., and Song, H. (2016). Mammalian Brain.

Bonaguidi, M.M.A., Wheeler, M.M.A.M., Shapiro, J.J.S.J., Stadel, R.P., Sun, G.J., Ming, G.L., and Song, H. (2011). In vivo clonal analysis reveals self-renewing and multipotent adult neural stem cell characteristics. Cell 145, 1142-1155.

Bond, A.M., Ming, G.-L., and Song, H. (2015). Cell Stem Cell Adult Mammalian Neural Stem Cells and Neurogenesis: Five Decades Later. Stem Cell 17, 385-395.

de Cabo, R., Carmona-Gutierrez, D., Bernier, M., Hall, M.N., and Madeo, F. (2014). The search 
for antiaging interventions: from elixirs to fasting regimens. Cell 157, 1515-1526.

Chandel, N.S., Jasper, H., Ho, T.T., and Passegué, E. (2016). Metabolic regulation of stem cell function in tissue homeostasis and organismal ageing. Nat. Cell Biol. 18, 823-832.

Chavali, M., Klingener, M., Kokkosis, A.G., Garkun, Y., Felong, S., Maffei, A., and Aguirre, A. (2018). Non-canonical Wnt signaling regulates neural stem cell quiescence during homeostasis and after demyelination. Nat. Commun. 9, 36.

Choi, S.H., Bylykbashi, E., Chatila, Z.K., Lee, S.W., Pulli, B., Clemenson, G.D., Kim, E., Rompala, A., Oram, M.K., Asselin, C., et al. (2018). Combined adult neurogenesis and BDNF mimic exercise effects on cognition in an Alzheimer's mouse model. Science (80-. ). 361, eaan8821.

Clevers, H. (2015). What is an adult stem cell? Science (80-. ). 350, 1319-1320.

Codega, P., Silva-Vargas, V., Paul4, A., Maldonado-Soto, A.R., DeLeo, A.M., Pastrana1, E., and Doetsch, F. (2015). Prospective identification and purification of quiescent adult neural stem cells from their in vivo niche. Neuron 82, 545-559.

Dobin, A., Davis, C.A., Schlesinger, F., Drenkow, J., Zaleski, C., Jha, S., Batut, P., Chaisson, M., and Gingeras, T.R. (2013). STAR: ultrafast universal RNA-seq aligner. Bioinformatics 29, 15-21.

Dranovsky, A., Picchini, A.M., Moadel, T., Sisti, A.C., Yamada, A., Kimura, S., Leonardo, E.D., and Hen, R. (2011). Experience Dictates Stem Cell Fate in the Adult Hippocampus. Neuron 70, 908-923.

Dulken, B.W., Leeman, D.S., Boutet, S.C., Hebestreit, K., and Brunet, A. (2017). Single-Cell Transcriptomic Analysis Defines Heterogeneity and Transcriptional Dynamics in the Adult Neural Stem Cell Lineage. Cell Rep. 18, 777-790.

Ehm, O., Göritz, C., Covic, M., Schäffner, I., Schwarz, T.J., Karaca, E., Kempkes, B., Kremmer, E., Pfrieger, F.W., and Espinosa, L. (2010). RBPJK-dependent signaling is essential for longterm maintenance of neural stem cells in the adult hippocampus. J. Neurosci. 30, 13794-13807. Encinas, J.M., Vaahtokari, A., and Enikolopov, G. (2006). Fluoxetine targets early progenitor cells in the adult brain. Proc. Natl. Acad. Sci. 103, 8233-8238.

Encinas, J.M., Michurina, T.V., Peunova, N., Park, J.-H., Tordo, J., Peterson, D.A., Fishell, G., Koulakov, A., and Enikolopov, G. (2011). Division-Coupled Astrocytic Differentiation and AgeRelated Depletion of Neural Stem Cells in the Adult Hippocampus. Cell Stem Cell 8, 566-579. Fan, X., Wheatley, E.G., and Villeda, S.A. (2017). Mechanisms of Hippocampal Aging and the Potential for Rejuvenation. Annu. Rev. Neurosci. 40, 251-272.

Fisher, R. (1956). The mathematics of a lady tasting tea. World Math. 
Gage, J.T., Vance, E.A., Hildenbrand, P.G., and Mattison, T. (2000). Brain lesion and AIDS. Proc. (Bayl. Univ. Med. Cent). 13, 424-429.

Gillespie, D.T. (1977). Concerning the validity of the stochastic approach to chemical kinetics. J. Stat. Phys. 16, 311-318.

Gillman, M.W. (2005). Developmental origins of health and disease. N. Engl. J. Med. 353, 1848-1850.

Gontier, G., Iyer, M., Shea, J.M., Bieri, G., Wheatley, E.G., Ramalho-Santos, M., and Villeda, S.A. (2018). Tet2 Rescues Age-Related Regenerative Decline and Enhances Cognitive Function in the Adult Mouse Brain. Cell Rep. 22, 1974-1981.

Goodell, M.A., and Rando, T.A. (2015). Stem cells and healthy aging. Science (80-. ). 350, 1199-1204.

Hagihara, H., Toyama, K., Yamasaki, N., and Miyakawa, T. (2009). Dissection of Hippocampal Dentate Gyrus from Adult Mouse. J. Vis. Exp.

Hattiangady, B., and Shetty, A.K. (2008). Aging does not alter the number or phenotype of putative stem/progenitor cells in the neurogenic region of the hippocampus. Neurobiol. Aging 29, 129-147.

Hayes, N.L., and Nowakowski, R.S. (2002). Dynamics of cell proliferation in the adult dentate gyrus of two inbred strains of mice. Brain Res. Dev. Brain Res. 134, 77-85.

Heine, V.M., Maslam, S., Joëls, M., and Lucassen, P.J. (2004). Prominent decline of newborn cell proliferation, differentiation, and apoptosis in the aging dentate gyrus, in absence of an agerelated hypothalamus-pituitary-adrenal axis activation. Neurobiol. Aging 25, 361-375.

Hochberg, Y., and Benjamini, Y. (1990). More powerful procedures for multiple significance testing. Stat. Med.

Hodge, R.D., Kowalczyk, T.D., Wolf, S.A., Encinas, J.M., Rippey, C., Enikolopov, G., Kempermann, G., and Hevner, R.F. (2008). Intermediate Progenitors in Adult Hippocampal Neurogenesis: Tbr2 Expression and Coordinate Regulation of Neuronal Output. J. Neurosci. 28, 3707-3717.

Igel, C., Suttorp, T., and Hansen, N. (2006). A Computational Efficient Covariance Matrix Update and a (1+1)-CMA for Evolution Strategies.

Jang, M.H., Bonaguidi, M.A., Kitabatake, Y., Sun, J., Song, J., Kang, E., Jun, H., Zhong, C., Su, Y., Guo, J.U., et al. (2013). Secreted frizzled-related protein 3 regulates activity-dependent adult hippocampal neurogenesis. Cell Stem Cell 12, 215-223.

Jones, K.M., Sarić, N., Russell, J.P., Andoniadou, C.L., Scambler, P.J., and Basson, M.A. (2015). CHD7 maintains neural stem cell quiescence and prevents premature stem cell 
depletion in the adult hippocampus. Stem Cells 33, 196-210.

Jongbloets, B.C., Lemstra, S., Schellino, R., Broekhoven, M.H., Parkash, J., Hellemons, A.J.C.G.M., Mao, T., Giacobini, P., van Praag, H., De Marchis, S., et al. (2017). Stage-specific functions of Semaphorin7A during adult hippocampal neurogenesis rely on distinct receptors. Nat. Commun. 8, 14666.

Kalamakis, G., Brüne, D., Ravichandran, S., Bolz, J., Fan, W., Ziebell, F., Stiehl, T., CataláMartinez, F., Kupke, J., Zhao, S., et al. (2019). Quiescence Modulates Stem Cell Maintenance and Regenerative Capacity in the Aging Brain. Cell 176, 1407-1419.e14.

Katsimpardi, L., and Lledo, P.-M. (2018). Regulation of neurogenesis in the adult and aging brain. Curr. Opin. Neurobiol. 53, 131-138.

Kennedy, B.K., Berger, S.L., Brunet, A., Campisi, J., Cuervo, A.M., Epel, E.S., Franceschi, C., Lithgow, G.J., Morimoto, R.I., Pessin, J.E., et al. (2014). Geroscience: Linking Aging to Chronic Disease. Cell 159, 709-713.

Keyes, B.E., and Fuchs, E. (2018). Stem cells: Aging and transcriptional fingerprints. J. Cell Biol. 217, 79-92.

Kim, E.J., Ables, J.L., Dickel, L.K., Eisch, A.J., and Johnson, J.E. (2011). Ascl1 (Mash1) defines cells with long-term neurogenic potential in subgranular and subventricular zones in adult mouse brain. PLoS One 6, e18472.

Kim, K.-S., Seeley, R.J., and Sandoval, D.A. (2018). Signalling from the periphery to the brain that regulates energy homeostasis. Nat. Rev. Neurosci. 19, 185-196.

Kirschen, G.W., Ge, S., and Snyder, J.S. (2019). Young at heart: Insights into hippocampal neurogenesis in the aged brain. Trends Neurosci. 369, 164-178.

Knoth, R., Singec, I., Ditter, M., Pantazis, G., Capetian, P., Meyer, R.P., Horvat, V., Volk, B., and Kempermann, G. (2010). Murine features of neurogenesis in the human hippocampus across the lifespan from 0 to 100 years. PLoS One 5, e8809.

Kuhn, H.G., Dickinson-Anson, H., and Gage, F.H. (1996). Neurogenesis in the dentate gyrus of the adult rat: age-related decrease of neuronal progenitor proliferation. J. Neurosci. 16, 20272033.

Kuhn, H.G., Toda, T., and Gage, F.H. (2018). Adult Hippocampal Neurogenesis: A Coming-ofAge Story. J. Neurosci. 38, 10401-10410.

Lê, S., Josse, J., and Husson, F. (2008). FactoMineR: an R package for multivariate analysis. J. Stat. Softw. 25, 1-18.

Leeman, D.S., Hebestreit, K., Ruetz, T., Webb, A.E., McKay, A., Pollina, E.A., Dulken, B.W., Zhao, X., Yeo, R.W., Ho, T.T., et al. (2018). Lysosome activation clears aggregates and 
enhances quiescent neural stem cell activation during aging. Science (80-. ). 359, 1277-1283. Lemberger, T., Parlato, R., Dassesse, D., Westphal, M., Casanova, E., Turiault, M., Tronche, F., Schiffmann, S.N., and Schütz, G. (2007). Expression of Cre recombinase in dopaminoceptive neurons. BMC Neurosci. 8, 4.

Li, A., and Horvath, S. (2007). Network neighborhood analysis with the multi-node topological overlap measure. Bioinformatics 23, 222-231.

Li, B., and Dewey, C.N. (2011). RSEM: accurate transcript quantification from RNA-Seq data with or without a reference genome. 12, 323.

Li, Y., Li, Y., McKay, R.M., Riethmacher, D., and Parada, L.F. (2012). Neurofibromin modulates adult hippocampal neurogenesis and behavioral effects of antidepressants. J. Neurosci. 32, 3529-3539.

Licht, T., Rothe, G., Kreisel, T., Wolf, B., Benny, O., Rooney, A.G., Ffrench-Constant, C., Enikolopov, G., and Keshet, E. (2016). VEGF preconditioning leads to stem cell remodeling and attenuates age-related decay of adult hippocampal neurogenesis. Proc. Natl. Acad. Sci. U. S. A. 113, E7828-E7836.

Limke, T.L., Rao, M.S., and Limke, T. (2002). Neural stem cells in aging and disease.

J.Cell.Mol.Med 6, 475-496.

Liu, L., Cheung, T.H., Charville, G.W., Hurgo, B.M.C., Leavitt, T., Shih, J., Brunet, A., and Rando, T.A. (2013). Chromatin modifications as determinants of muscle stem cell quiescence and chronological aging. Cell Rep. 4, 189-204.

Llorens-Bobadilla, E., Zhao, S., Baser, A., Saiz-Castro, G., Zwadlo, K., and Martin-Villalba, A. (2015). Single-Cell Transcriptomics Reveals a Population of Dormant Neural Stem Cells that Become Activated upon Brain Injury. Cell Stem Cell 17, 329-340.

López-Otín, C., Blasco, M.A., Partridge, L., Serrano, M., and Kroemer, G. (2013). The Hallmarks of Aging. Cell 153, 1194-1217.

Lugert, S., Vogt, M., Tchorz, J.S., Müller, M., Giachino, C., and Taylor, V. (2012). Homeostatic neurogenesis in the adult hippocampus does not involve amplification of Ascl1 high intermediate progenitors. Nat. Commun. 3.

Mahmoudi, S., Xu, L., and Brunet, A. (2019). Turning back time with emerging rejuvenation strategies. Nat. Cell Biol. 21, 32-43.

La Manno, G., Soldatov, R., Zeisel, A., Braun, E., Hochgerner, H., Petukhov, V., Lidschreiber, K., Kastriti, M.E., Lönnerberg, P., Furlan, A., et al. (2018). RNA velocity of single cells. Nature 560, 494-498.

Mattson, M.P., Pedersen, W.A., Duan, W., Culmsee, C., and Camandola, S. (1999). Cellular 
and molecular mechanisms underlying perturbed energy metabolism and neuronal degeneration in Alzheimer's and Parkinson's diseases. Ann. N. Y. Acad. Sci. 893, 154-175. Moreno-Jiménez, E.P., Flor-García, M., Terreros-Roncal, J., Rábano, A., Cafini, F., PallasBazarra, N., Ávila, J., and Llorens-Martín, M. (2019). Adult hippocampal neurogenesis is abundant in neurologically healthy subjects and drops sharply in patients with Alzheimer's disease. Nat. Med. 25, 554-560.

Mu, Y., and Gage, F.H. (2011). Adult hippocampal neurogenesis and its role in Alzheimer's disease. Mol. Neurodegener. 6, 85.

Muzumdar, M.D., Tasic, B., Miyamichi, K., Li, L., and Luo, L. (2007). A global double-fluorescent Cre reporter mouse. Genesis 45, 593-605.

Obernier, K., and Alvarez-Buylla, A. (2019). Neural stem cells: origin, heterogeneity and regulation in the adult mammalian brain. Development 146, dev156059.

Ogrodnik, M., Zhu, Y., Langhi, L.G.P., Tchkonia, T., Krüger, P., Fielder, E., Victorelli, S., Ruswhandi, R.A., Giorgadze, N., Pirtskhalava, T., et al. (2019). Obesity-Induced Cellular Senescence Drives Anxiety and Impairs Neurogenesis. Cell Metab. 29, 1061-1077.e8. Oh, J., Lee, Y.D., and Wagers, A.J. (2014). Stem cell aging: Mechanisms, regulators and therapeutic opportunities. Nat. Med. 20, 870-880.

Pan, Y.-W., Zou, J., Wang, W., Sakagami, H., Garelick, M.G., Abel, G., Kuo, C.T., Storm, D.R., and Xia, Z. (2012). Inducible and conditional deletion of extracellular signal-regulated kinase 5 disrupts adult hippocampal neurogenesis. J. Biol. Chem. 287, 23306-23317.

Peng, L., and Bonaguidi, M.A. (2018). Function and Dysfunction of Adult Hippocampal Neurogenesis in Regeneration and Disease. Am. J. Pathol. 188, 23-28.

Pilz, G.-A., Bottes, S., Betizeau, M., Jörg, D.J., Carta, S., Simons, B.D., Helmchen, F., and Jessberger, S. (2018). Live imaging of neurogenesis in the adult mouse hippocampus. Science 359, 658-662.

Renault, V.M., Rafalski, V.A., Morgan, A.A., Salih, D.A.M., Brett, J.O., Webb, A.E., Villeda, S.A., Thekkat, P.U., Guillerey, C., Denko, N.C., et al. (2009). FoxO3 Regulates Neural Stem Cell Homeostasis. Cell Stem Cell 5, 527-539.

Ritchie, M.E., Phipson, B., Wu, D., Hu, Y., Law, C.W., Shi, W., and Smyth, G.K. (2015). Limma powers differential expression analyses for RNA-sequencing and microarray studies. Nucleic Acids Res.

Robinson, M.D., McCarthy, D.J., and Smyth, G.K. (2010). edgeR: a Bioconductor package for differential expression analysis of digital gene expression data. Bioinformatics.

Sahin, E., and DePinho, R.A. (2010). Linking functional decline of telomeres, mitochondria and 
stem cells during ageing. Nature 464, 520-528.

Schloerke, B., Crowley, J., Cook, D., Hofmann, H., Wickham, H., Briatte, F., and Marbach, M. (2014). GGally: Extension to ggplot2. R Packag. Version 0.4.6. Http//CRAN.R-

Project.Org/Package=GGally.

Sean, D., and Meltzer, P.S. (2007). GEOquery: A bridge between the Gene Expression Omnibus (GEO) and BioConductor. Bioinformatics.

Shin, J.Y.Y.J., Berg, D.A.A., Zhu, Y., Shin, J.Y.Y.J., Song, J., Bonaguidi, M.A.A., Enikolopov, G., Nauen, D.W.W., Christian, K.M.M., Ming, G.L., et al. (2015). Single-Cell RNA-Seq with Waterfall Reveals Molecular Cascades underlying Adult Neurogenesis. Cell Stem Cell 17, 360372.

Sierra, A., Martín-Suárez, S., Valcárcel-Martín, R., Pascual-Brazo, J., Aelvoet, S.-A., Abiega, O., Deudero, J.J., Brewster, A.L., Bernales, I., Anderson, A.E., et al. (2015). Neuronal Hyperactivity Accelerates Depletion of Neural Stem Cells and Impairs Hippocampal Neurogenesis. Cell Stem Cell 16, 488-503.

Solanas, G., Peixoto, F.O., Perdiguero, E., Jardí, M., Ruiz-Bonilla, V., Datta, D., Symeonidi, A., Castellanos, A., Welz, P.-S., Caballero, J.M., et al. (2017). Aged Stem Cells Reprogram Their Daily Rhythmic Functions to Adapt to Stress. Cell 170, 678-692.e20.

Soneson, C., and Robinson, M.D. (2018). Bias, robustness and scalability in single-cell differential expression analysis. Nat. Methods 15, 255-261.

Song, J., Zhong, C., Bonaguidi, M.A., Sun, G.J., Hsu, D., Gu, Y., Meletis, K., Huang, Z.J., Ge, S., Enikolopov, G., et al. (2012). Neuronal circuitry mechanism regulating adult quiescent neural stem-cell fate decision. Nature 489, 150-154.

Southall, T.D., Gold, K.S., Egger, B., Davidson, C.M., Caygill, E.E., Marshall, O.J., and Brand, A.H. (2013). Cell-type-specific profiling of gene expression and chromatin binding without cell isolation: Assaying RNA pol II occupancy in neural stem cells. Dev. Cell 26, 101-112.

Spalding, K.L., Bergmann, O., Alkass, K., Bernard, S., Salehpour, M., Huttner, H.B., Boström, E., Westerlund, I., Vial, C., Buchholz, B.A., et al. (2013). Dynamics of Hippocampal Neurogenesis in Adult Humans. Cell 153, 1219-1227.

Sun, D., Luo, M., Jeong, M., Rodriguez, B., Xia, Z., Hannah, R., Wang, H., Le, T., Faull, K.F., Chen, R., et al. (2014). Epigenomic profiling of young and aged HSCs reveals concerted changes during aging that reinforce self-renewal. Cell Stem Cell 14, 673-688.

Toda, T., and Gage, F.H. (2018). Review: adult neurogenesis contributes to hippocampal plasticity. Cell Tissue Res. 373, 693-709.

Torres-Pérez, M., Tellez-Ballesteros, R.I., Ortiz-López, L., Ichwan, M., Vega-Rivera, N.M., 
Castro-García, M., Gómez-Sánchez, A., Kempermann, G., and Ramirez-Rodriguez, G.B. (2015). Resveratrol Enhances Neuroplastic Changes, Including Hippocampal Neurogenesis, and Memory in Balb/C Mice at Six Months of Age. PLoS One 10, e0145687. van Velthoven, C.T.J., and Rando, T.A. (2019). Stem Cell Quiescence: Dynamism, Restraint, and Cellular Idling. Cell Stem Cell 24, 213-225.

Wang, J.Y.J. (2014). The Capable ABL: What Is Its Biological Function? Mol. Cell. Biol. 34, 1188-1197.

Wong, S.T., Trinh, K., Hacker, B., Chan, G.C.K., Lowe, G., Gaggar, A., Xia, Z., Gold, G.H., and Storm, D.R. (2000). Disruption of the Type III Adenylyl Cyclase Gene Leads to Peripheral and Behavioral Anosmia in Transgenic Mice. Neuron 27, 487-497.

Zhu, Y., Tchkonia, T., Pirtskhalava, T., Gower, A.C., Ding, H., Giorgadze, N., Palmer, A.K., Ikeno, Y., Hubbard, G.B., Lenburg, M., et al. (2015). The Achilles' heel of senescent cells: from transcriptome to senolytic drugs. Aging Cell 14, 644-658.

Ziebell, F., Dehler, S., Martin-Villalba, A., and Marciniak-Czochra, A. (2018). Revealing agerelated changes of adult hippocampal neurogenesis using mathematical models. Development 145, dev153544.

(2001). High Precision Stereotaxic Surgery in Mice. 


\begin{tabular}{|l|l|l|}
\hline Adobe Illustrator & Adobe.com & N/A \\
\hline Imaris 8.0 & Bitplane & N/A \\
\hline STAR & (Dobin et al., 2013) & N/A \\
\hline RSEM & (Li and Dewey, 2011) & N/A \\
\hline edgeR & (Robinson et al., 2010) & N/A \\
\hline topGO & $\begin{array}{l}\text { (Alexa A and } \\
\text { Rahnenfuhrer J, 2016) }\end{array}$ & N/A \\
\hline limma & (Ritchie et al., 2015) & N/A \\
\hline RNA velocity & (La Manno et al., 2018) & N/A \\
\hline Other & & \\
\hline Osmotic pump & Alzet & Cat\#: 1007D \\
\hline
\end{tabular}

Contact for Reagent and Resource Sharing

Further information and requests for resources and reagents should be directed to and will be fulfilled by the Lead Contact: Michael A. Bonaguidi (mbonagui@usc.edu )

\section{Experimental Model and Subject Details}

Animals and Tamoxifen Administration

All animal procedures were performed in accordance with institutional guidelines of University of Southern California Keck School of Medicine and protocol (20287) approved by Institutional Animal Care and Use Committee (IACUC). All mice used in the study were backcrossed to the C57BL/6 background to ensure the reproducibility of clonal induction with specific doses of tamoxifen. Animals were housed in a 12-hour light/12hour dark cycle with free access to food.

Nestin-CreER ${ }^{\mathrm{T} 2}$ mice (Balordi and Fishell, 2007) and Ascl1-CreER ${ }^{\mathrm{T} 2}$ mice (Kim et al., 2011) were used to clonally label RGLs. The following genetically modified mice were originally purchased from Jackson Labs: Rosa-YFPf/f (Strain: B6.129X1$\left.\mathrm{Gt}(\mathrm{ROSA})^{26 S o r t m 1(E Y F P) C o s} / \mathrm{J}\right), \mathrm{mT} / \mathrm{mG}^{\mathrm{fff}}$ (Strain: B6.129(Cg)-Gt(ROSA)26Sortm4(ACTB-tdTomato,-

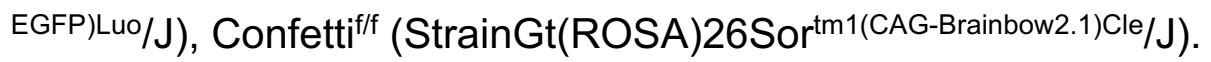

Nestin-CreER ${ }^{\mathrm{T} 2}$ and Ascl1-CreER ${ }^{\mathrm{T} 2}$ mice were crossed to fluorescent reporter mice for clonal analysis. Nestin-CreER ${ }^{\top 2}::$ Confetti $^{f /+}$ mice were generated by breeding

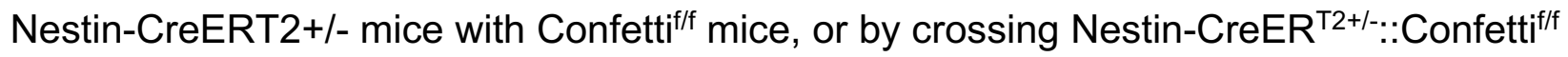

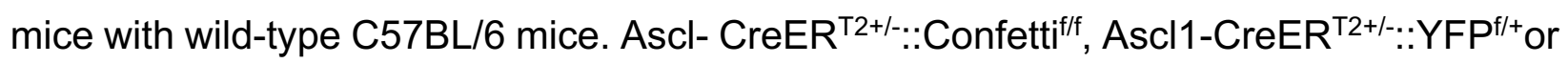
Ascl1-CreER ${ }^{\mathrm{T} 2+/}:: \mathrm{mTmG}^{\mathrm{f} /+}$ mice were generated by crossing Ascl1-CreER ${ }^{\mathrm{T} 2+/}$ with Confettif/f, $\mathrm{YFP}^{\mathrm{f} / \mathrm{f}}$ or $\mathrm{mTmG}^{\mathrm{f} / \mathrm{f}}$, respectively. For each CreER ${ }^{\mathrm{T} 2}$ driver, we tested different 
tamoxifen doses and reporter lines, and obtained combinations that exhibited high specificity, inducibility and reproducibility (Table S1, S3-S4).

At least 3 animals were checked for each reporter/driver combination to ensure there was no recombination in the adult SGZ in the absence of tamoxifen. A stock of tamoxifen $(66.6 \mathrm{mg} / \mathrm{ml})$ was prepared in a $5: 1$ ratio of corn oil to ethanol at $37^{\circ} \mathrm{C}$ with occasional vortexing. A single tamoxifen or vehicle dose was intraperitoneally injected into 8- to 10-week-old, 26- to 30-week-old, or 52-week-old mice at various concentrations for lineage tracing. Injected animals showed no signs of distress.

Primer sets from original publications were used to identify genetically modified mice (Ahn and Joyner, 2005; Balordi and Fishell, 2007; Kim et al., 2011; Lemberger et al., 2007; Muzumdar et al., 2007) (Table S3). Genomic tail DNA was isolated in a 25mM $\mathrm{NaOH}, 0.2 \mathrm{mM}$ EDTA solution and run for 35 PCR cycles.

\section{Method details}

Immunostaining, Confocal Imaging, and Processing

Mice were anesthetized with isoflurane gas and underwent transcardial perfusion with saline followed by $4 \%$ paraformaldehyde. Brains were post-fixed overnight in $4 \%$ paraformaldehyde and then immersed in $30 \%$ sucrose for a subsequent 48 hours prior to sectioning. Brains were sectioned into $45 \mu \mathrm{m}$ coronal sections through the entire dentate gyrus. Immunohistology was performed with antibodies as previously described (Bonaguidi et al., 2011)(Bonaguidi et al., 2011)(Bonaguidi et al., 2011)(Bonaguidi et al., 2011)(Bonaguidi et al., 2011)(Bonaguidi et al., 2011) on sections in serial order using custom, in-house staining chambers. Brain sections were washed in TBS with $0.3 \%$ Triton-X100 prior to staining and mounting. Goat anti-GFP (1:1000), rabbit anti-RFP (1:1000), rabbit anti-GFAP (1:2000), chicken anti-Nestin (1:500), mouse anti-MCM2 (1:500) primary antibodies were used. Antigen retrieval for MCM2 and Nestin antibodies utilized DAKO citrate buffer at $95^{\circ} \mathrm{C}$ for $20 \mathrm{~min}$ and then left to cool at room temperature. Cells were then counted using every 8th section throughout the entire dentate $(n=6-7$ sections per dentate) for a stereological analysis and every section was processed for clonal analysis. GFP+ cells were identified with a Zeiss AxioObserver.A1 microscope and 
were acquired as a z-stack on a Zeiss LSM700 confocal system under 40x or 63x magnification. Morphological analysis was done using Imaris 8.0 Software.

Clonal Analysis

Clonal analysis and categorization of cell types by morphological and immunohistological criteria were consistent with prior criteria (Bonaguidi et al., 2011). Analyzed dentate volume included the stratum granulosum (granule cell layer), and SGZ. Serial sections were first screened for candidate clones, which were defined as possessing at least (1) an RGL, (2) neuronal cell(s) in close spatial proximity, or (3) astroglia in close spatial proximity to other astroglia or neuronal cells. Approximately 618 clones per dentate per color (in the case of Confetti) allowed for clonal analysis based on prior computer simulations (Bonaguidi et al., 2011) (Table S4). Clones were randomly induced throughout the dentate.

Dividing clones were marked with two separate nuclei by DAPI under confocal microscopy. The two nuclei were completely encompassed when using membrane-bound reporters (Rosa-CFP, $\mathrm{mT} / \mathrm{mG}$, Confetti-CFP), or completely filled when using cytoplasmic-bound reporters (Rosa-YFP, Rosa-RFP, Confetti-YFP, Confetti-RFP).

Clones were categorized according to the clone composition among RGL-containing clones (Bonaguidi et al., 2011). Clones with more than one RGL and progeny were classified by the lineage produced: neuronal, astroglial, or both. RGL maintenance was assessed as a percentage of clones that contained at least one RGL.

Stereotaxic surgery (Imatinib infusion via osmotic pump)

Stereotaxic surgery was performed on 10-months-old C57/BL6 mice as described (Pan et al., 2012; 2001; Wong et al., 2000). Mice were anesthetized using an isoflurane machine (5\% until recumbent, 2-3\% maintenance). Prior to anesthesia, non-steroidal antiinflammatory analgesic - $1 \mathrm{X}$ Ketoprofen $(5 \mathrm{mg} / \mathrm{kg}$ ) was injected. Osmotic pump with the drug (1 mM Imatinib) or 1\% DMSO vehicle control was unilaterally implanted at a rate of $0.5 \mu \mathrm{l} / \mathrm{hr}$ for 6 days (Figure $3 \mathrm{~K}$ ) into the hippocampal fimbria with the following coordinates relative to Bregma: $-0.8 \mathrm{~mm}$ posterior, $-0.75 \mathrm{~mm}$ medial-lateral, and $2.5 \mathrm{~mm}$ ventral. 
Fluorescence- activated Cell Sorting (FACs) of Individual cells from Adult Mouse Dentate Gyrus

Homozygous Nestin:CFPnuc mice (Encinas et al., 2006) were used for all singlecell RNA-seq experiments (Shin et al., 2015). Mice were euthanized by cervical dislocation, and brains were immediately immersed into cold Hibernate A solution (BrainBits). Dentate gyri were dissected under a stereomicroscope as previously described (Hagihara et al., 2009). All procedures were performed with approved protocols in accordance with institutional animal guidelines. The single cell suspension was prepared by using Neural Tissue Dissociation Kit $(P)$ (Miltenyi Biotech), with the addition of one cleaning step with Percoll (1:10 dilution) to remove myelin layer and cellular debris. Propidium lodide was added to determine cell viability and cells were sorted on a BD FACs Aria II with a $70 \mu \mathrm{m}$ nozzle at 13 psi. (Fig. S3) Single cells were collected onto the ice-cold 8-sample parafilm-covered glass slides with $1.25 \mathrm{uL}$ of $1 \mathrm{X}$ lysis mixture (Clontech Laboratories SMART-Seq v4 Ultra Low Input RNA Kit for sequencing) and immediately transferred in individual 0.2-ml RNase-free 8-well strip (Figure S3). To avoid any effects due to the FACs sorting protocol, cells were kept on ice the entire procedure, and the actual collection was performed at $4^{\circ} \mathrm{C}$.

scRNA-seq library preparation and sequencing

cDNA was generated using the SMART-Seq v4 Ultra Low Input RNA Kit (Clontech Laboratories) for sequencing with modifications to bring down cost. Briefly, all reagents from the kit (for Reverse transcription and cDNA amplification) were miniaturized 10-fold by utilizing FACs collection in a very low volume of the lysis buffer. The same quality of cDNA was achieved as for the original kit. The amplification product was purified using Ampure XP beads according to the manufacturer's protocol. Quality control and validation were performed by using qPCR for Actin expression and Agilent Tape Station 4200 (Agilent Technologies).

The amplification product was sheared using library preparation Nextera XT Kit (Illumina Inc.) following the manufacturer's protocol. In brief, the purified cDNA was tagmented and fragmented using a miniaturized protocol. cDNA fragments were barcoded using i5 and i7 indices (IDT) and the product was purified using Ampure RNA 
clean beads. The purified ligation product underwent 13 cycles of PCR. A Mantis Liquid Handler (Formulatrix, Inc.) was used to handle low volume pipetting. The libraries were multiplexed and sequenced using Illumina NextSeq 550 (Illumina) 75 paired end run. To minimize the batch effect on the sequencing all cells were loaded into one chip.

\section{Preprocessing, Alignment and Count Estimation of RNA-Seq data}

FASTQ files were demultiplexed into two cell-specific FASTQ files by i5 and i7 adapter sequences for forward and reverse ends of the paired-end reads. Raw reads were trimmed of TSO sequence and adaptor contaminants using Trimmomatic (Bolger et al., 2014). Sequences were then aligned using STAR (Dobin et al., 2013) to the mm10 mouse transcriptome, constructed using UCSC annotation framework, generating cellspecific BAM files. Only uniquely mapped, concordant reads were preserved. RSEM (Li and Dewey, 2011) was then employed to produce spliceoform-level count estimates, which were summated to give gene-level counts per cell. Cells with fewer than 150,000 uniquely mapped, concordant reads were excluded. Remaining cells then underwent transcripts per million (TPM) normalization (Li and Dewey, 2011) to control for variable gene length within samples and sequencing depth between samples. Cells with fewer than 1,000 detected genes were also excluded, where a gene was considered detected in a cell if $>2$ TPM. After cell exclusions, 48 cells from 2 month-old mice and 41 cells from 4.5 month-old mice remained, totaling 89 cells. 3,603 genes were detected on average $(\mathrm{SD}=833)$.

\section{Clustering, Dimensionality Reduction and RNA Velocity}

K-means clustering, employing the Hartigan-Wong algorithm, was run on all 89 cells in full 24,411-dimensional expression space. K was set equal to 5, as Shin et al.(Shin et al., 2015) described 5 clusters present in adult neural stem cells. Dimensionality reduction of 89 cells was performed by PCA, using the FactoMineR (Lê et al., 2008) package with default parameters.

RNA Velocity(La Manno et al., 2018) was used to estimate the future expression state of single cells. Count estimates of unspliced and spliced cells were computed with the following command velocityto run_smartseq2 $-d \quad 1 \quad \$ b a m \_f i l e s$ 
\$ucsd_annotation_mm10.gtf. Unspliced and spliced count estimates were imported to the gene.relative.velocity.estimate() function to produce future expression state estimates for every cell. Future expression states were represented in previously computed PCA embedding as arrows.

To represent the extent to which aging dampens the tendency for cells to proceed down the neurogenic trajectory, we visualized the future state vectors of each age group at each cluster. Vectors were centered on the mean coordinate within their respective cluster in PC space. The $\mathrm{X}$ corresponds to PC1 coordinate space, where $\mathrm{Y}$ corresponds to PC2 coordinate space. First mean, coordinates for each cluster were derived. Cells within any one cluster were computed thusly -

$$
\begin{aligned}
& \bar{x}_{1}=\frac{\sum_{i=1}^{N} x_{1 i}}{N} \\
& \bar{y}_{1}=\frac{\sum_{i=1}^{N} y_{1 i}}{N}
\end{aligned}
$$

Future state predictions were then aligned atop each other at the mean coordinate by -

$$
\begin{gathered}
\hat{x}=x_{2}-x_{1}+\bar{x}_{1} \\
\hat{y}=y_{2}-y_{1}+\bar{y}_{1}
\end{gathered}
$$

This computation was run independently on cells from the 2-month-old and 4.5-monthold groups to produce single cell estimates.

\section{Differential expression, niche comparisons and Gene Ontology enrichment}

Differential expression on Nestin:CFP ${ }^{+}$scRNA-Seq generated for this paper was computed using the edgeR package in $R$ (Robinson et al., 2010). Specifically, we employed the general liner model quasi likelihood fit test edgeR (edgeRQLFDetRate), as it was optimal across all major evaluation criteria in a recent survey of contemporary differential expression methods developed for single cell RNA Sequencing (Soneson and Robinson, 2018). In all use cases, age group was the only factor for the generalized linear model, where there were two age groups (2-month-old and 4.5-month-old). Gene filtering 
is required for accurate implementation of edgeR. Only genes with a mean count greater than 5 in at least one of the age groups were included any analysis. P-values were groupadjusted using the Benjamini-Hochberg (Hochberg and Benjamini, 1990) method to produce a false detection rate (FDR). Genes with an FDR <=0.05 were preserved for downstream analyses.

Transcriptomes from epidermal stem cell (Solanas et al., 2017) and muscle stem cell (Liu et al., 2013) isolations were observed on Affymetrix Mouse Gene microarrays. Both datasets were obtained via GEOquery (Sean and Meltzer, 2007) using the getGEO() function. To obtain differentially expressed gene sets between young and aged cells, samples were first log transformed and quantile normalized. Expression units were then fit to a linear model where age was the single factor. Finally, an empirical Bayes statistical test was run on each bead using default parameters of the eBayes() function in the limma package (Ritchie et al., 2015). P-values were group-adjusted using the BenjaminiHochberg (Hochberg and Benjamini, 1990) method to produce a false detection rate (FDR). As some beads encompassed multiple genes, gene ID names were disaggregated to create a differentially expressed gene set of all gene names implicated by the empirical Bayes test. Differentially expressed genes between young and aged hematopoietic stem cells were obtained from tests run by (Sun et al., 2014).

To determine if the convergence between the early aging expression signature observed in NSCs and the more advanced aging signature of other somatic stem cells was statistically significant, we ran a Fisher's exact test (Fisher, 1956) comparing the genes differentially upregulated in NSC aging against the genes differentially upregulated with aging in each of the other stem cell niches against a gene universe encompassing genes jointly present on the Affymetrix microarray and UCSC genome annotation. The same series of Fisher's tests was run on downregulated genes.

TopGO (Alexa A and Rahnenfuhrer J, 2016) was employed to derive enriched Gene Ontology terms associated with aging in each stem cell niche. Two ontology enrichment lists were generated for each niche - one from upregulated genes and another from downregulated genes. Enrichment was calculated using the classic Fisher's test (Fisher, 1956), where exact p-values were corrected using Benjimini-Hochberg (Hochberg and Benjamini, 1990). 
As was performed at the level of genes, Gene Ontology (GO)-level convergence in aging signatures between NSCs and other stem cell niches was also examined. A Fisher's exact test was employed between upregulated NSC GO terms and the upregulated GO terms in each of the other niches. Downregulated terms were similarly examined.

\section{String network visualization}

To represent connectivity between genes and GO terms associated with NSC aging, we wrote custom functions to visualize a string network. The underlying object for this network is represented as the bipartite graph $G=(\mathrm{V}, \mathrm{E})$ where, $\mathrm{V}(G)$ is a set of vertices, which can be partitioned into two independent sets, GO terms $A$, and genes $B$. To reduce visual complexity, we generated the subgraph $H$ where $\mathrm{V}(G) \subseteq \mathrm{V}(H)$ and $\mathrm{E}(G) \subseteq \mathrm{E}(H)$. The minimum allowable edges is $a$, thus conditionally $\delta(H)=a$. Construction of graphs connecting genes to both GO terms and niches is tripartite yet follows the same design as the previous bipartite graph, with the addition of a new subset to $\mathrm{V}(G)$. Once constructed, graphs were matricized and visualized as a string network using force-directed Kamadakawai spatial organization in the GGally package(Schloerke et al., 2014).

\section{Computational modeling}

To consolidate the discussion in the main text, in the following we further explain the fitting and modelling approaches used.

\section{Distribution of activation times}

For the Figure 2E, the activation time of a radial glial-like NSC (RGL) is the time until it first enters cell cycle upon labelling ( $\left.T_{\text {entry }}\right)$. To deduce the distribution of activation times from the clonal data, we calculated at each time point the fraction of RGLs that had not yet divided. 
As the labelling protocols targeted a small proportion of IPCs in addition to RGLs, there was a degree of error involved in our assignment: we could not decide unambiguously whether a clone consisting entirely of IPCs and differentiated cell types was originally derived from an RGL or an IPC. In the case of Ascl1\#-RGL clonal data, the number of such clones was so small that their exclusion would not significantly affect our results. For Nestin\#-RGL, 10/34 clones consist only of 1-2 IPCs at 2 dpi; we assumed that these were IPC-derived and excluded them from the analysis. By $7 \mathrm{dpi}$, due to the short cell cycle time of IPCs (Berg et al., 2015; Hayes and Nowakowski, 2002; Hodge et al., 2008), we would expect IPC-derived clones to have grown in size; we therefore took clones containing 6 or more IPCs to be IPC-derived (2/40), and clones consisting only of 2 IPCs to be RGL-derived (4/40). At later time points, we assumed that all clones were RGL-derived. As a second caveat, a clone consisting of a single RGL was scored as an undivided RGL but may have given rise to progeny that were subsequently lost through cell death. However, effects of any erroneous assignments were likely negligible compared to experimental noise over this timescale.

\section{Cell cycle re-entry times}

For Figure 2F we can estimate cell cycle re-entry times from the fraction of cells that have divided exactly once. For a population of cells with activation rate $\lambda$ and cell cycle re-entry rate $\mu$, the fraction of cells divided once at time $t$, denoted $R^{1}(t)$, satisfies

$$
\frac{d R^{1}(t)}{d t}=\lambda \cdot R^{0}(t)-\mu \cdot R^{1}(t)
$$

where $R^{0}(t)=e^{-\lambda t}$ is the fraction of cells that remain undivided at time $t$.

Multiplying by the integrating factor $e^{\mu t}$ yields

$$
e^{\mu t} \cdot \frac{d R^{1}(t)}{d t}=\lambda \cdot e^{(\mu-\lambda) t}-\mu \cdot e^{\mu t} \cdot R^{1}(t)
$$

with the solution $R^{1}(t)=\frac{\lambda}{\mu-\lambda} \cdot \frac{e^{(\mu-\lambda) t}+C}{e^{\mu t}}$, where $C$ is a constant. Since $R^{1}(0)=0$, we must have $C=-1$ and therefore $R^{1}(t)=\frac{\lambda}{\mu-\lambda} \cdot \frac{e^{(\mu-\lambda) t}-1}{e^{\mu t}}=\frac{\lambda}{\mu-\lambda} \cdot\left(e^{-\lambda t}-e^{-\mu t}\right)$.

Given our estimates of the activation times, weighted least-squared fits suggested cell time to the first Nestin\# - RGL division for 2 months old is $23 \pm 3$ days, for 6 months 
old is $38 \pm 3$ days; cycle re-entry times for 2 months old is $40 \pm 3$ and for 6 months old is $100 \pm 4$ days (Figure 2D-F).

Model of a developmental-like stem cell fate program (ST - Ascl1\#-NSC). Here we introduce a simplified Markovian model that captures the progression of radial-glia like neural stem cells (NSCs) through a developmental-like programme that consists of a proliferative phase, followed by a neurogenic phase and terminal differentiation (Pilz et al., 2018). In our model, NSCs divide with a transition rate $\lambda$ (corresponding to an average cycle time $T=\lambda^{-1}$ ) and go through $R_{0}$ rounds of duplication (symmetric self-renewing divisions), followed by $R_{1}$ rounds of asymmetric division, generating a certain amount of neuronal progeny upon each division, followed by $R_{2}=1$ round of terminal differentiation. During each differentiating division, the amount of neuronal progeny generated (interpreted as containing all cell types down the neuronal lineage) is exponentially distributed with a mean proliferative output $m_{N}$. This procedure takes into account amplification by intermediate progenitors. Neuronal progeny dies at a rate $\omega_{N}$. Initially, a clone starts as a single NSC (with probability $q$ ) with uniform probability to find it at any state within the programme or a neuronal progenitor/neuron (with probability $1-q$ ).

Formally, the state of a clone is given by the vector $(\boldsymbol{r}, n)=$ $\left(r_{0,0}, \ldots, r_{0, R_{0}-1}, r_{0,1}, \ldots, r_{0, R_{1}-1}, r_{2,0}, n\right)$, where $r_{\sigma, i}$ is the number of NSCs that are in phase $\sigma$, where $\sigma=0$ corresponds to the proliferative phase, $\sigma=1$ corresponds to the phase of asymmetric neurogenic divisions and $\sigma=2$ corresponds to the phase of terminal differentiation, and $i$ is the number of divisions that a cell has gone through in the respective phase; $n$ denotes the neuronal content. The dynamics of the probability $P=$ $P(\boldsymbol{r}, n, t)$ to find a clone with configuration $(\boldsymbol{r}, n)$ at time $t$ is described by the master equation

$$
\frac{\partial P}{\partial t}=\left\{\lambda \mathbb{E}_{0}\left(R_{0}\right)+\lambda \mathbb{E}_{1}\left(R_{1}\right)+\lambda \mathbb{E}_{2}(1)+\omega_{N} \mathbb{D}\right\} P
$$

where we have defined the following operators: $\mathbb{E}_{\sigma}(R)$ describes the progression through the phase $\sigma=0,1,2$ of the developmental-like programme, 


$$
\begin{aligned}
\mathbb{E}_{\sigma}(R) & =\sum_{i=0}^{R-2}\left[\left(r_{\sigma, i}+1\right) \mathbb{R}_{\sigma, i}^{+}\left(\mathbb{R}_{\sigma, i+1}^{-}\right)^{2-\sigma} \mathbb{P}_{N}\left(m_{N}\right)^{\sigma}-r_{\sigma, i}\right] \\
& +\left(r_{\sigma, R-1}+1\right) \mathbb{R}_{\sigma, R-1}^{+}\left(\mathbb{R}_{\sigma+1,0}^{-}\right)^{2-\sigma} \mathbb{P}_{N}\left(m_{N}\right)^{\sigma}-r_{\sigma, R-1}
\end{aligned}
$$

where the $\mathbb{R}_{\sigma, i}^{ \pm}$are ladder operators that increase/decrease the number of RGLs at stage $(\sigma, i)$ according to $\mathbb{R}_{\sigma, i}^{ \pm} P\left(\ldots, r_{\sigma, i}, \ldots\right)=P\left(\ldots, r_{\sigma, i} \pm 1, \ldots\right)$ and

$$
\mathbb{P}_{N}(m)=\sum_{k=0}^{\infty} \varepsilon_{k}(m)\left(\mathbb{N}^{-}\right)^{k}
$$

is the operator that generates neuronal progeny with an exponentially distributed number of newborn cells with average $m$ with $\varepsilon$ being a discrete exponential distribution; here, $\mathbb{N}^{ \pm}$ are the ladder operators for the variable $n$. Furthermore, we define the operator describing death of neuronal progeny as

$$
\mathbb{D}=(n+1) \mathbb{N}^{+}-n
$$

Here, we impose the boundary condition $P(\boldsymbol{r}, n, t)=0$ if $r_{\sigma, i}<0$ for at least one $(\sigma, i)$. The initial condition described above is formally given by $P(\boldsymbol{r}, n, 0)=q\left(R_{0}+R_{1}+R_{2}\right)^{-1}+(1-$ q) $\delta_{n, 1} \delta_{r_{\sigma, i}}$

Motivated by the fact that Ascl1 ${ }^{\#}$-targeted cells seem to be primed for entry into cycle (see main text), we allow the first division to occur at a faster rate $\lambda_{1}=\alpha \lambda$, where $\alpha>1$ indicates the fold-change as compared to the re-entry rate $\lambda$. Formally, this entails a straightforward modification of Eq. (1), which we omit here since it obscures the basic features of the stochastic dynamics. The model is solved numerically using a standard stochastic simulation algorithm of the Gillespie-type (Gillespie, 1977).

To describe the clonal dynamics of the Ascl1"-targeted population, we identify model parameters using the following strategy. First, all parameters are determined 
simultaneously by comparing the model to the 2-months age dataset, fitting the timedependent average clone content $\bar{r}$, the time-dependent 'RGL survival' $\bar{s}$, defined as fraction of clones containing at least one RGL (Figure S2) and the average number $\bar{r}_{*}$ of RGLs per RGL-containing clone,

$$
\begin{gathered}
\bar{r}(t)=\sum_{\sigma, i} \frac{\left\langle r_{\sigma, i}(t)\right\rangle}{P_{*}(t)}, \quad \bar{n}(t)=\frac{\langle n(t)\rangle}{P_{*}(t)}, \\
\bar{s}(t)=\frac{1-\sum_{n} P(\mathbf{0}, n, t)}{P_{*}(t)}, \quad \bar{r}_{*}(t)=\sum_{\sigma, i} \frac{\left\langle r_{\sigma, i}(t)\right\rangle}{1-\sum_{n} P(\mathbf{0}, n, t)},
\end{gathered}
$$

where $P_{*}(t)=1-P(\mathbf{0}, 0, t)$ is the total surviving fraction of clones. For successive later ages, all parameters are held constant except for the neuronal death rate $\omega_{N}$, which is determined individually for each age by refitting the model to the respective dataset. To implement this fit strategy, we compute the sum of squared differences between experimental data points and simulations for each of these quantitites individually and then obtain a combined cost function $R$ by multiplying these individual residuals,

$$
\begin{aligned}
R(\boldsymbol{\theta})= & \left(\sum_{i}\left(\bar{r}_{\text {sim }}\left(t_{i} \mid \boldsymbol{\theta}\right)-\bar{r}_{\text {exp }}\left(t_{i}\right)\right)^{2}+\sum_{i}\left(\bar{n}_{\text {sim }}\left(t_{i} \mid \boldsymbol{\theta}\right)-\bar{n}_{\text {exp }}\left(t_{i}\right)\right)^{2}\right) \\
& \times\left(\sum_{i}\left(\bar{s}_{\text {sim }}\left(t_{i} \mid \boldsymbol{\theta}\right)-\bar{s}_{\text {exp }}\left(t_{i}\right)\right)^{2}\right)\left(\sum_{i}\left(\bar{r}_{\text {sim }}^{*}\left(t_{i} \mid \boldsymbol{\theta}\right)-\bar{r}_{\text {exp }}^{*}\left(t_{i}\right)\right)^{2}\right)
\end{aligned}
$$

where $\boldsymbol{\theta}$ is the respective set of parameters and $t_{i}$ are the experimentally available time points. The cost function $R$ is minimized using a stochastic optimization algorithm (a Covariance Matrix Adaptation Evolution Strategy using the cma-es Python package (Igel et al., 2006). All fit parameters are found in Figure S2.

\section{Model of cell fate dynamics within a small stem cell niche}

In this model, NSCs divide within a small niche region that can harbor a maximum of $\mathcal{N}$ NSCs. NSCs stochastically divide at a rate $\lambda$. If the niche is not maximally occupied, NSCs duplicate with probability $p$ and differentiate with probability $1-p$ upon which NSCs are lost while generating a certain amount of neuronal and glial progeny. As in the model of the developmental-like programme, the amount of progeny generated (interpreted as containing all cell types down the neuronal or glial lineage, respectively) is exponentially 
distributed with a mean proliferative output $m_{N}$ or $m_{G}$, respectively. If the niche is maximally occupied, the duplication channel is blocked and cells differentiate with probability 1. Neuronal and glial progeny dies at rates $\omega_{N}$ and $\omega_{G}$, respectively. The state of a clone is given by the vector $(r, n, g)$ where $r$ is the number of NSCs, $n$ denotes the neuronal content and $g$ denotes the glial content. Initially, the clone starts as a single NSC. The dynamics of the probability $P=P(r, n, g, t)$ to find a clone with configuration $(r, n, g)$ at time $t$ is described by the master equation

$$
\begin{gathered}
\frac{\partial P}{\partial t}=\left\{p \lambda(r-1) \mathbb{R}^{-}+\left(1-p+p \delta_{r+1, \mathcal{N}}\right) \lambda(r+1) \mathbb{R}^{+} \mathbb{P}_{N}\left(m_{N}\right) \mathbb{P}_{G}\left(m_{G}\right)\right. \\
\left.-\lambda r+\omega_{N} \mathbb{D}_{N}+\omega_{G} \mathbb{D}_{G}\right\} P,
\end{gathered}
$$

where the operators $\mathbb{P}_{x}$ and $\mathbb{D}_{x}$ being defined analogously to Eqs. (3) and (4) with $\mathbb{P}_{G}$ given by $\mathbb{P}_{N}$ with $\mathbb{N}^{ \pm}$being replaced by the ladder operator $\mathbb{G}^{ \pm}$for the glial progeny number $g$. Here we impose the boundary conditions $P(-1, t)=P(\mathcal{N}+1, t)=0$, which also ensure that the niche occupation cannot exceed its maximum capacity $\mathcal{N}$. As for the developmental-like model, we add the possibility for the first division to occur at a faster rate $\lambda_{1}=\alpha \lambda$.

Parameters are determined using the following strategy. First, all parameters are determined in the same way described in the previous section for the developmental-like programme (Figure S2). Cycle times at later ages are inferred from the total population data, an independent data set (Figure S2). To this end, we fit the total RGL volume $V(t)$ by a function of the form $V(t)=V_{0}+V_{1} e^{-k t}$ and assume that the time evolution of the RGL loss rate $\eta$, defined by $\dot{V}=-\eta V$, reflects changes in cycle time. Hence, we infer relative changes in the cycle time at later age by the factor

$$
\eta(t)=-\frac{d \ln V}{d t}=\frac{k}{1+\left(V_{0} / V_{1}\right) e^{k t}}
$$

so that

$$
\lambda_{6 m}=\frac{\eta(6 m)}{\eta(2 m)} \lambda_{2 m}, \quad \lambda_{12 m}=\frac{\eta(12 m)}{\eta(6 m)} \lambda_{6 m}
$$


Using the thus inferred cycle rates and the other fit parameters, only the neuronal and glial death rates are determined using refits at 6 months of age. All fit parameters are found in Table S2.

\section{Statistics}

For in vivo experiments, the number of independent experimental replicates are indicated in figure legends, with $\mathrm{n}$ representing $\mathrm{n}$ experimental replicates using at least $\mathrm{n}$ animals. For single cell RNA-seq experiment, 2-3 mice per group were used to obtain enough cells. Unpaired student's t-tests were used to generate p-values for data comparison by using Prism software. All clones observed at each time point were treated as statistically equivalent. No randomization or blinding was used in the animal studies. Mouse dentate gyri with exceedingly low $(<6)$ or high $(>18)$ clones per color (Confetti) per hemisphere were omitted from the study. Sample sizes were estimated in accordance to prior clonal studies (Bonaguidi et al., 2011; Song et al., 2012). Error bars in the study represent the standard errors in mean frequencies, calculated as $\sqrt{ }(p(1-p) / N)$ where $p$ is the frequency of a given characteristic and $\mathrm{N}$ the number of clones considered. Mathematical modeling was performed by weighted least squares using custom-written MATLAB scripts. Representative images are depicted in Figures: 1, S1-S3. A pairedsamples t-test was conducted to compare the ability of Imatinib to activate NSCs against batch-matched vehicle controls. There was a significant difference in the activation rates for Imatinib-treated ( $\mathrm{M}=25.84 \%, \mathrm{SD}=7.77 \%)$ against batch-matched vehicle controls $(\mathrm{M}=18.29 \%, \mathrm{SD}=2.45 \%) ; \mathrm{t}(5)=2.09 \mathrm{p}=0.045$ related to Figure $3 \mathrm{~L}-\mathrm{N}$. 


\section{Supplementary Tables:}

Table S1. Tamoxifen doses used to achieve clonal recombination among various promoter, reporter and ages contexts over the analyzed time course, related to Figures 1-3.

\begin{tabular}{|c|c|c|c|c|c|}
\hline Promoter & Age & Reporter & [Tamoxifen] & Chase & Figure(s) \\
\hline Ascl1::CreER ${ }^{\top 2}$ & $2 \mathrm{mo}$ & $\begin{array}{l}\text { ROSA- } \\
\text { YFP }\end{array}$ & $78 \mathrm{mg} / \mathrm{kg}$ & $3,7,30 \mathrm{dpi}$ & 1 \\
\hline Ascl1::CreER ${ }^{\top 2}$ & $2 \mathrm{mo}$ & $\mathrm{mT} / \mathrm{mG}$ & $124-217 \mathrm{mg} / \mathrm{kg}$ & $3,7 \mathrm{dpi}$ & 1,2 \\
\hline Ascl1::CreER ${ }^{\top 2}$ & $6 \mathrm{mo}$ & $\begin{array}{l}\mathrm{mT} / \mathrm{mG} \\
\mathrm{ROSA}- \\
\text { YFP and } \\
\text { Confetti }\end{array}$ & 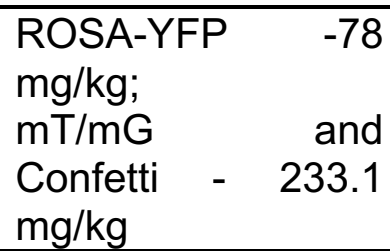 & $\begin{array}{l}3,7, \quad 30 \\
\text { dpi }\end{array}$ & 1,2 \\
\hline Nestin::CreER ${ }^{\top 2}$ & $2 \mathrm{mo}$ & Z/EG & $62 \mathrm{mg} / \mathrm{kg}$ & $\begin{array}{lr}2, & 7, \\
60, & 120 \\
\text { dpi } & \\
\end{array}$ & 1 \\
\hline Nestin::CreER ${ }^{\top 2}$ & $6 \mathrm{mo}$ & Confetti & $66.6 \mathrm{mg} / \mathrm{kg}$ & $\begin{array}{l}7,30,60 \\
\mathrm{dpi}\end{array}$ & 1,2 \\
\hline
\end{tabular}


Table S2. Parameter list and best fit parameters for the theoretical mode

\begin{tabular}{|c|c|c|}
\hline Parameter & Fit value & Description \\
\hline \multicolumn{3}{|c|}{ Ascl1-targeted population (developmental-like program model) } \\
\hline$\lambda$ & & cycle rate \\
\hline$T$ & & (corresponding average cycle time) \\
\hline$\lambda_{1}$ & & activation rate after induction \\
\hline$T_{\mathrm{A}}$ & & (corresponding activation time) \\
\hline \multicolumn{3}{|l|}{$R_{0}$} \\
\hline \multicolumn{3}{|l|}{$R_{1}$} \\
\hline$m_{\mathrm{N}}$ & & $\begin{array}{l}\text { avg. amount of neuronal progeny generated per } \\
\text { differentiating division }\end{array}$ \\
\hline$\omega_{\mathrm{N}}$ & & neuronal death rate \\
\hline$q$ & & $\begin{array}{l}\text { probability to induce an RGL (as compared to a more } \\
\text { differentiated cell) }\end{array}$ \\
\hline \multicolumn{3}{|c|}{ Nestin ${ }^{\#-t a r g e t e d ~ p o p u l a t i o n ~(m i c r o n i c h e ~ m o d e l) ~}$} \\
\hline$\lambda$ & & cycle rate \\
\hline$T$ & & (corresponding average cycle time) \\
\hline$\lambda_{1}$ & & activation rate after induction \\
\hline$T_{\mathrm{A}}$ & & (corresponding activation time) \\
\hline $\mathcal{N}$ & 2 & niche capacity \\
\hline$p$ & & $\begin{array}{l}\text { base probability for duplication (unless niche is fully } \\
\text { occupied) }\end{array}$ \\
\hline$m_{\mathrm{N}}$ & & $\begin{array}{l}\text { avg. amount of neuronal progeny generated per } \\
\text { differentiating division }\end{array}$ \\
\hline$m_{\mathrm{G}}$ & & $\begin{array}{l}\text { avg. amount of glial progeny generated per differentiating } \\
\text { division }\end{array}$ \\
\hline$\omega_{\mathrm{N}}$ & & neuronal death rate \\
\hline$\omega_{G}$ & & glial death rate \\
\hline$q$ & & $\begin{array}{l}\text { probability to induce an RGL (as compared to a more } \\
\text { differentiated cell) }\end{array}$ \\
\hline
\end{tabular}

Table S3. Primer sets that were used for genotyping

\begin{tabular}{|l|l|}
\hline Name & Primer (5'-3') \\
\hline NestinCreER_F & TGG CAG GAC ATG CTA CCT C \\
\hline NestinCreER_R & AGG CAA ATT TTG GTG TAC GG \\
\hline Ascl1_KI_F & AAC TTT CCT CCG GGG CTC GTT TC \\
\hline
\end{tabular}




\begin{tabular}{|l|l|}
\hline Ascl1_KI_R & CGC CTG GCG ATC CCT GAA CAT G \\
\hline Rosa10_F & CTC TGC TGC CTC CTG GCT TCT \\
\hline Rosa11_R & CGA GGC GGA TCA CAA GCA ATA \\
\hline Rosa4_R & TCA ATG GGC GGG GGT CGT T \\
\hline Confetti_R & CCA GAT GAC TAC CTA TCC TC \\
\hline Confetti_F_WT & CTC CTG GCT TCT GAG GAC C \\
\hline Confetti_F_spec & GAA TTA ATT CCG GTA TAA CTT CG \\
\hline MADM11_het-homo_F & TTC CCT TTC TGC TTC ATC TTG C \\
\hline MADM11_het-homo_R_WT & TGG AGG AGG ACA AAC TGG TCA C \\
\hline MADM11_het-homo_R_MUT & TCA ATG GGC GGG GGT CGT T \\
\hline
\end{tabular}


Table S4. Number of clones that for clonal analysis

Summary of number of clones across different ages and tracing timepoints for clonal analysis for Figures 1-3 and Supplementary Figures S1-S3

\begin{tabular}{|l|l|l|}
\hline Nestin\#-2mo & \# of all clones & \# of NSC clones \\
\hline $2 \mathrm{dpi}$ & 47 & 36 \\
\hline 7dpi & 40 & 34 \\
\hline $30 \mathrm{dpi}$ & 54 & 45 \\
\hline 60dpi & 98 & 66 \\
\hline 120dpi & 69 & 28 \\
\hline & & \\
\hline Nestin\#-6mo & & \\
\hline 7dpi & 68 & 44 \\
\hline 30dpi & 64 & 53 \\
\hline $60 \mathrm{dpi}$ & 53 & 46 \\
\hline & & \\
\hline Ascl1\#-2mo & & \\
\hline 3dpi & 83 & 61 \\
\hline 7dpi & 92 & 69 \\
\hline 30dpi & 70 & 46 \\
\hline & & \\
\hline Ascl1\#-6mo & & \\
\hline 3dpi & 63 & 30 \\
\hline 7dpi & 101 & 57 \\
\hline $30 \mathrm{dpi}$ & 78 & 20 \\
\hline
\end{tabular}




\section{Table S5. (Separate file)}

Quality metrics of RNA-seq libraries

Table S6. (Separate file)

Gene expression matrices

Table S7. (Separate file)

Differential gene expression of qNSC between young and old

Table S8. (Separate file)

GO term enrichment and gene presence matrix

Table S9. (Separate file)

Differential gene expression of comparative stem cell niches between young and aged

Table S10. (Separate file)

Upregulated GO term enrichment across stem cell niches and gene presence matrix

\section{Table S11. (Separate file)}

Downregulated GO term enrichment across stem cell niches and gene presence matrix

Table S12. (Separate file)

GO term co-enrichment tables

Codes for computational modeling and scRNA-seq analysis are available upon request. 
Figure 1

A
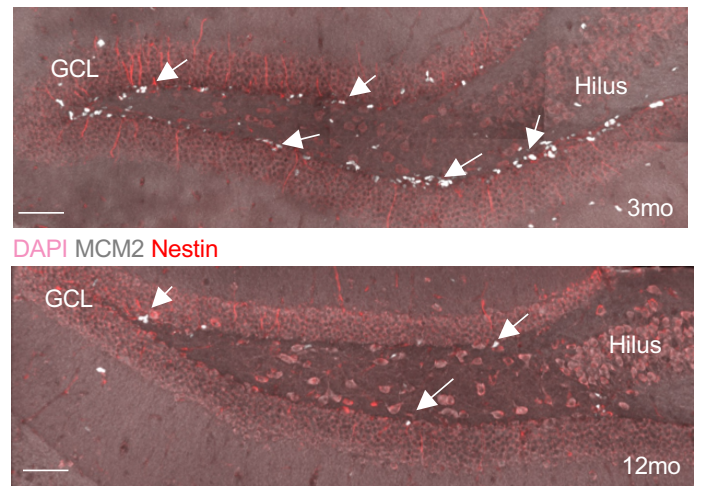

D
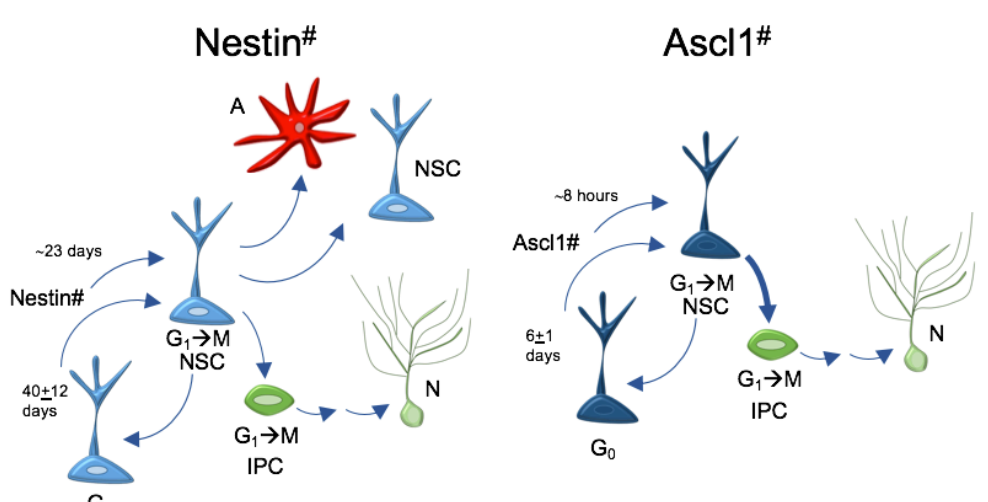

G

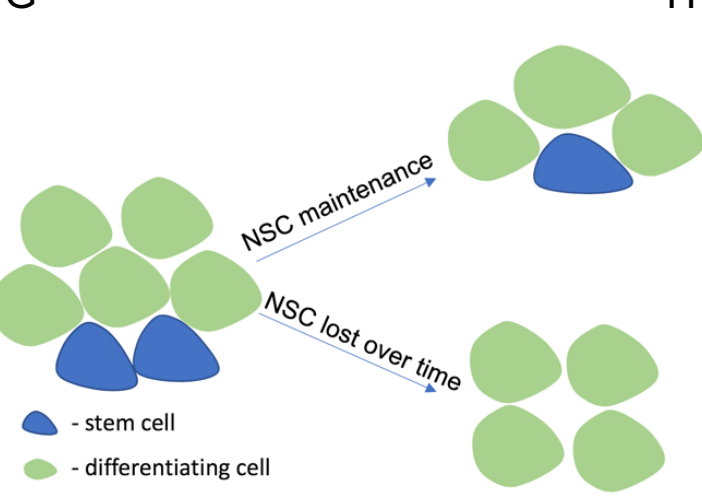

H

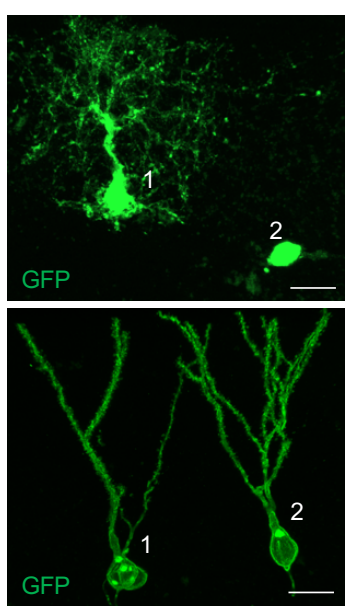

J

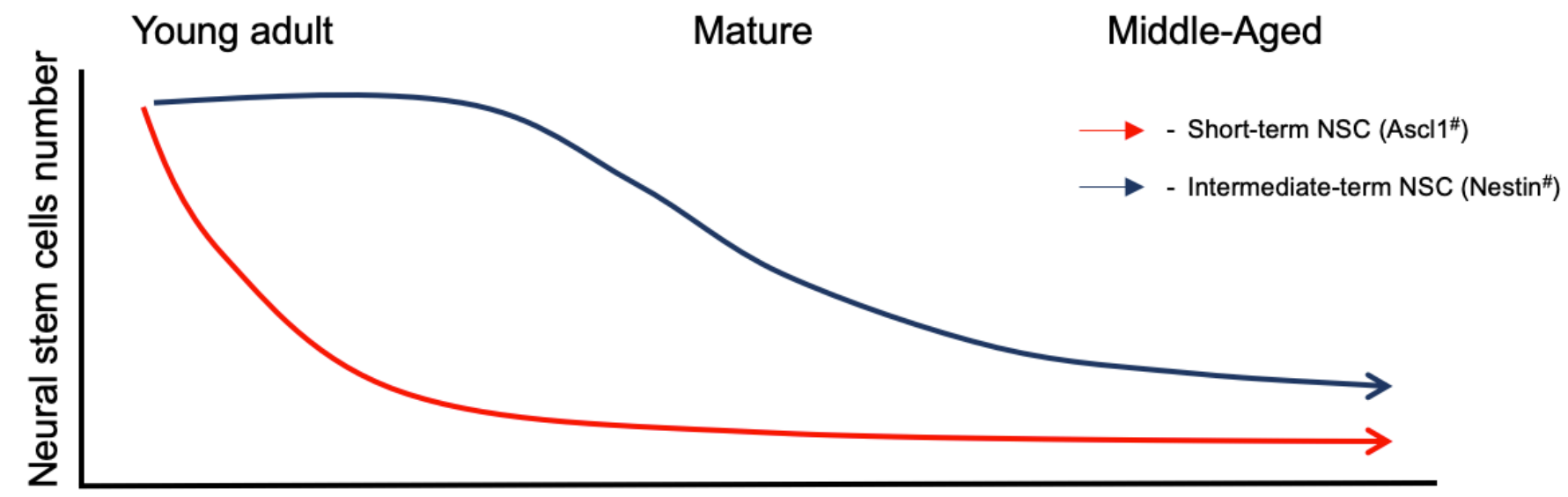

E

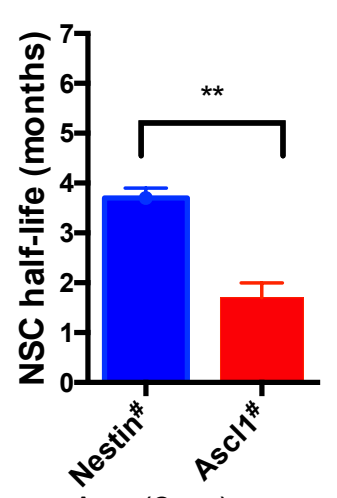

Age (2mo)

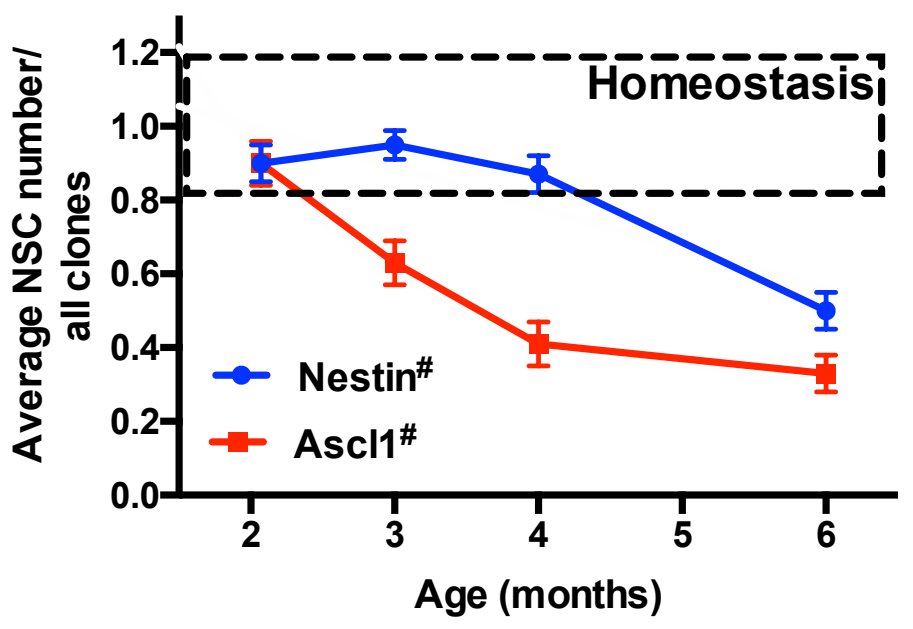

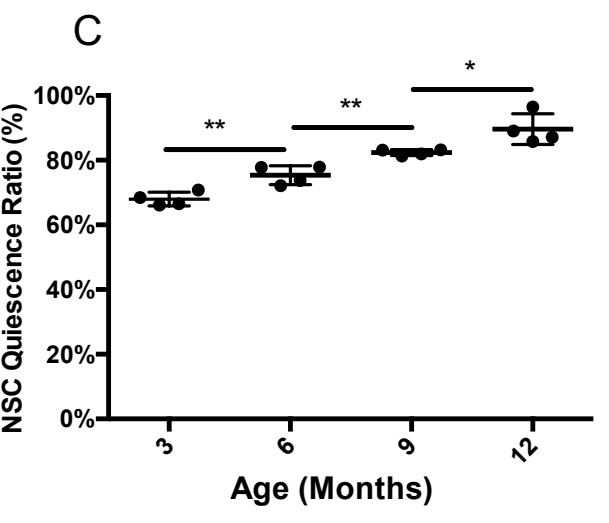

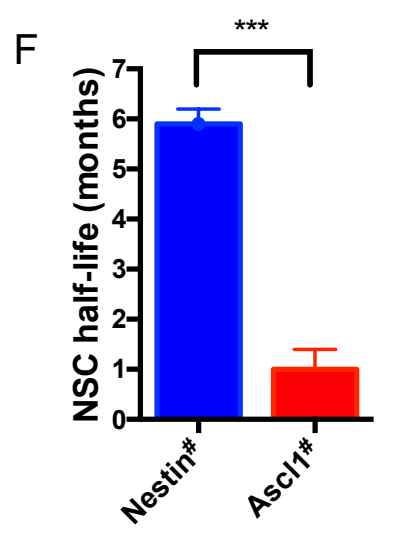

Age (6mo)

Aging 
Figure 1. Asynchronous neural stem cell (NSC) decline during aging.

(A) Sample confocal images showing immunofluorescence for NSCs (Nestin+) and cell proliferation $\left(\mathrm{Mcm}^{+}\right)$in the dentate gyrus of 3-month-old and 12-month-old mouse hippocampus. Scale bar, $100 \mu \mathrm{m}$

(B) Quantification of the total NSC number in the dentate gyrus across ages. $N=4-6$ mice; Values represent mean \pm SEM. ${ }^{*} p<0.05,{ }^{* *} p<0.01$; Student's t-test.

(C) Quantification of the percentage of quiescent NSCs (Nestin+ MCM2-) among total NSCs in the dentate gyrus across ages. $\mathrm{N}=4-6$ mice. Values represent mean $\pm \mathrm{SEM}$. ${ }^{*} \mathrm{p}<0.05,{ }^{* *} \mathrm{p}<0.01$; Student's t-test.

(D) Cartoon of NSC subpopulations in the adult hippocampus. (left) Nestin::CreER (Nestin\#)-labeled multipotential NSCs. (right) Developmental-like NSCs labeled by Ascl1::CreER (Ascl1\#). A=astroglial lineage, IPC=intermediate progenitor cell, $N=$ neuronal lineage, $G_{0}=$ quiescent state, $G_{1 \rightarrow M}=$ active state. Time (days) represent duration until state change.

(E) Nestin\#-NSCs display greater longevity (half-life) than Ascl1\#-NSCs in 2 month- old mice. $\mathrm{N}=20-101$ clones, see details in Table S4; Values represent mean \pm SEM ${ }^{* *} p<0.01$; Student's t-test.

(F) Nestin\#-NSCs display greater longevity (half-life) than Ascl1\#-NSCs in 6 month- old mice. N=20-101 clones, see details in Table S4; Values represent mean \pm SEM. ${ }^{* * *} p<0.001$; Student's t-test.

(G) Cartoon depicting clonal maintenance and depletion.

(H) Sample confocal images of a maintained clone containing a NSC (1) and intermediate progenitor cells (IPCs) (2); and a depleted clone containing neurons (1) and (2). Scale bar, $20 \mu \mathrm{m}$

(I) Quantification of NSC homeostasis duration. Ascl1\#-NSCs are rapidly depleted, while Nestin\#-NSCs maintain as stem cells for months before eventually differentiating. Values represent mean \pm SEM. $(N=20-$ 101 clones, see details in Table S4).

(I) Summary cartoon showing the asynchronous decline of NSCs during aging. 


\section{Figure 2} certified by peer review) is the author/funder. All rights reserved. No reuse allowed without permission.
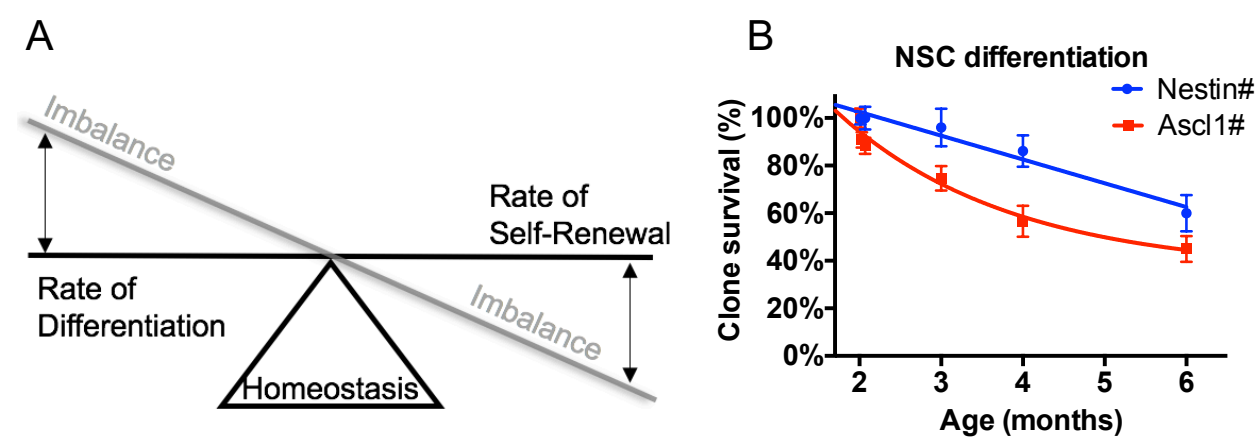

D

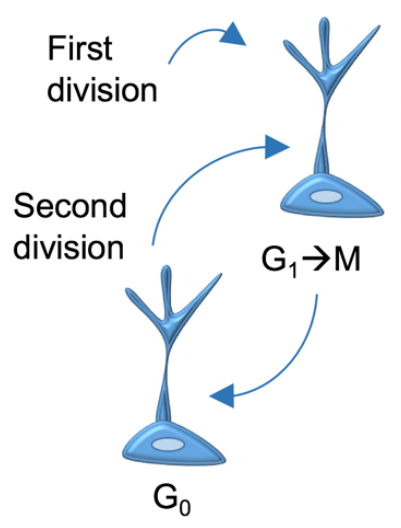

G
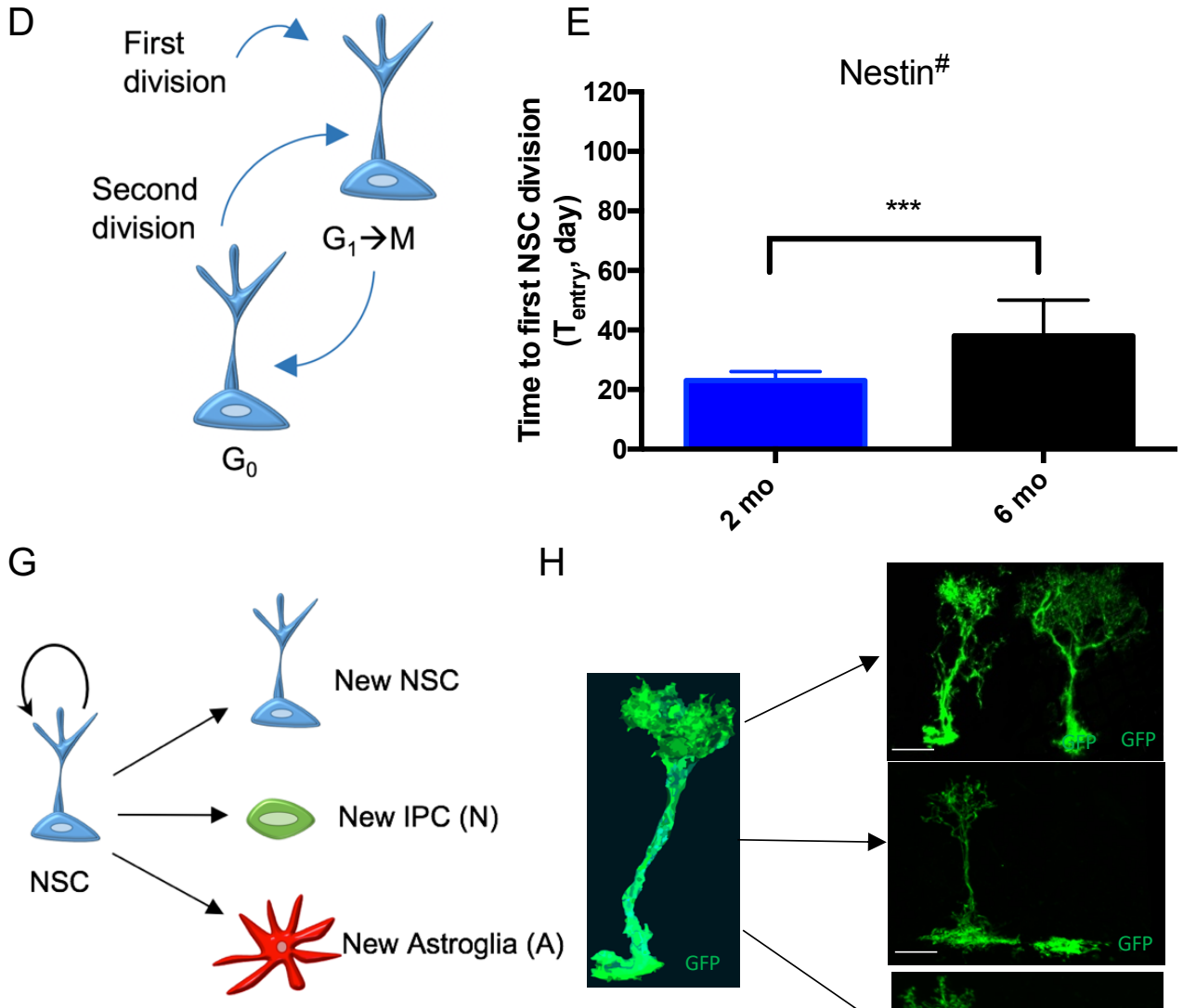

$\mathrm{H}$

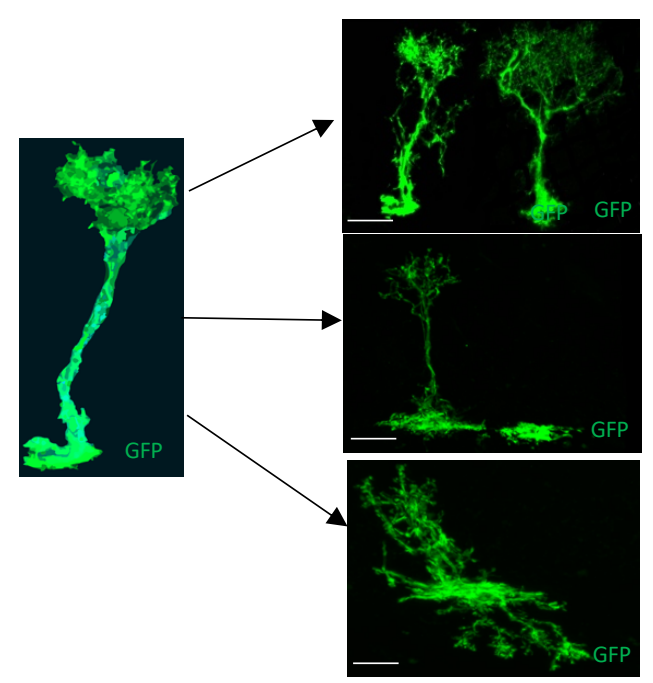

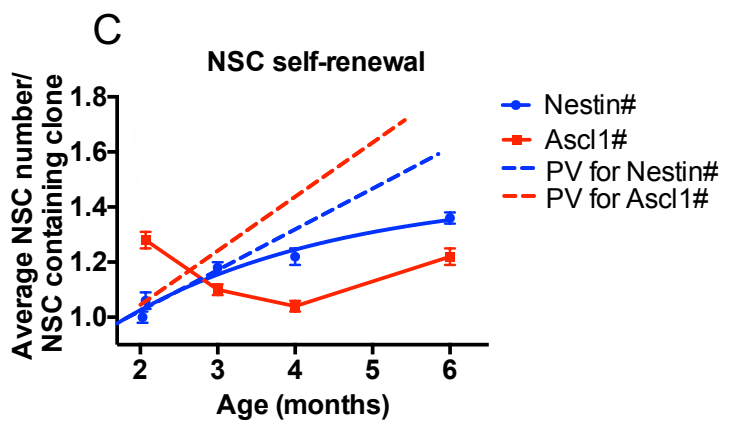

F

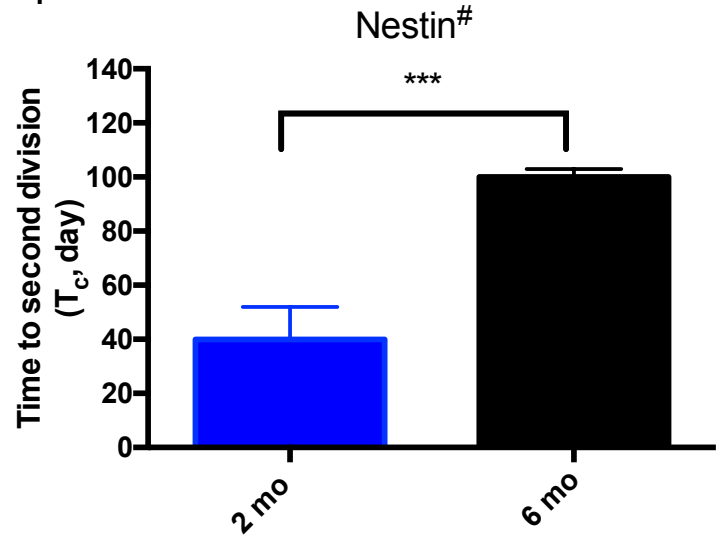

I

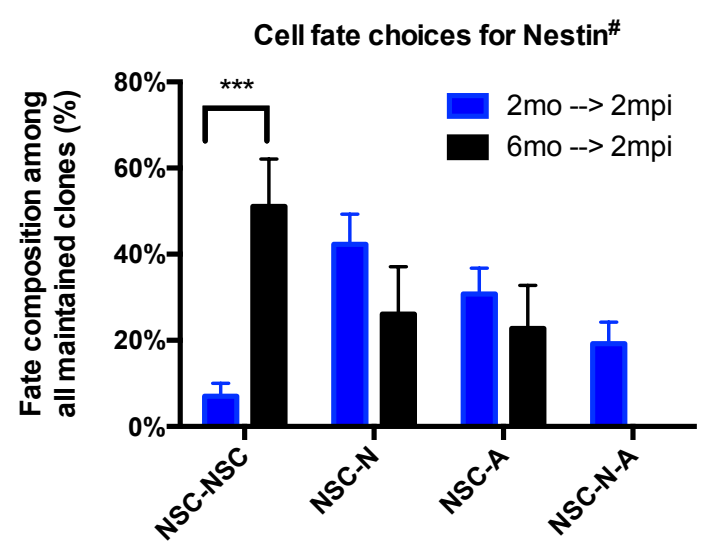

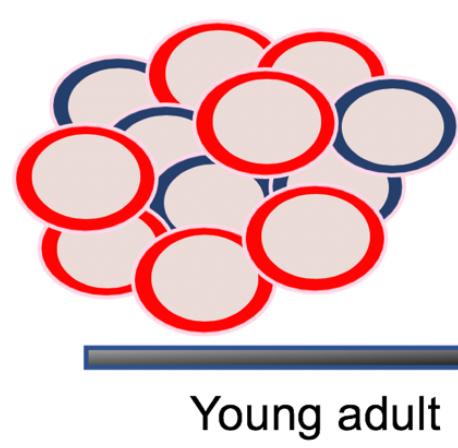
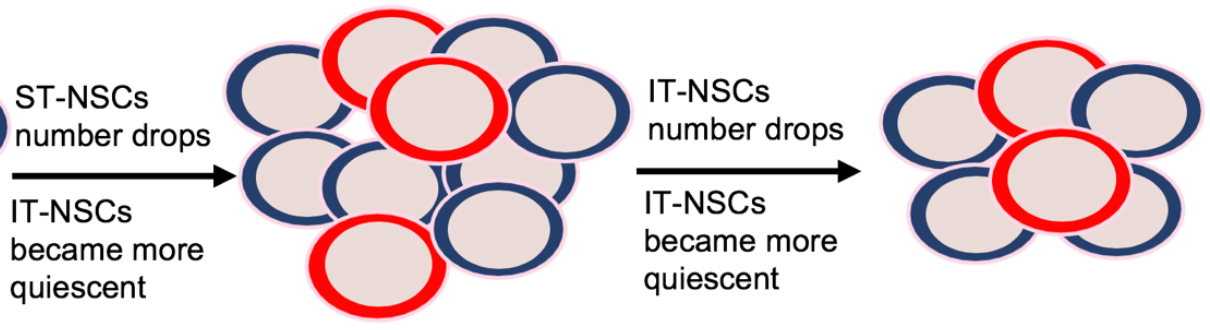

Mature

Middle-Aged 


\section{Figure 2. Increased quiescence drives NSC loss of homeostasis}

(A) Cartoon illustrating NSC homeostasis and its imbalance by changes in NSC expansion or depletion.

(B) Quantification of NSC differentiation in the mature hippocampus, Nestin\#-NSC clones are depleted at a constant rate. ( $\mathrm{N}=36-98$ clones, see details in Table S4).

(C) Quantification of NSC self-renewal in the mature hippocampus, Nestin\#-NSCs slow their expansion in approximately 4-month old mice indicating homeostatic imbalance. $\mathrm{PV}=$ predicted value to maintain NSC homeostasis. ( $\mathrm{N}=36-98$ clones, see details Table $\mathrm{S} 4)$.

(D) Cartoon of time to the first and second NSC divisions. ( $G_{0}=$ quiescence; $\left.G_{1} \rightarrow M=a c t i v a t i o n\right)$.

(E) Quantification of Nestin\#-NSC cell cycle entry based on lineage analysis from 2-month-old and 6month-old mice. Values represent mean \pm SEM. $\left(N=36-98\right.$ clones, see details Table S4; ${ }^{* *} p<0.001$; Student's t-test).

(F) Quantification of Nestin\#-NSC cell cycle re-entry based on lineage analysis from 2-month-old and 6month-old mice. Values represent mean \pm SEM ( $N=36-98$ clones, see details Table S4; ${ }^{* * *} p<0.001$; Student's t-test).

(G) Schematic illustration of NSC fate choices. NSC=neural stem cell; $A=$ astroglial lineage; IPC=intermediate progenitor cell ( $\mathrm{N}=$ neuronal lineage).

(H) Representative confocal images of clonal NSC fate choices. Scale bar, $10 \mu \mathrm{m}$.

(I) Quantifications of Nestin\#-NSCs fate choices based on single cell lineage tracing in 2-month-old and 6-month-old mice for 2 months post injection ( $60 \mathrm{dpi})$. Values represent mean $\pm \mathrm{SEM}(\mathrm{N}=36-66$ clones, see details Table S4; ${ }^{* * *} p<0.001$. Student's t-test).

(J) Cartoon summary of the cellular mechanisms driving age-related NSC dysfunction. 
Figure 3

A 2 mont Nestin:CFPnuc

4.5 month Nestin:CFPnuc
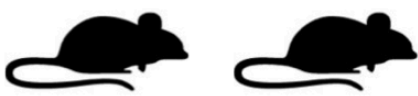

Microdissection

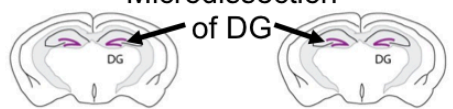

FACs sorting

Nestin:CFP+

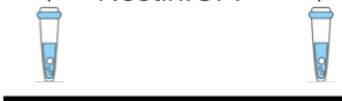

Single cell RNA-seq

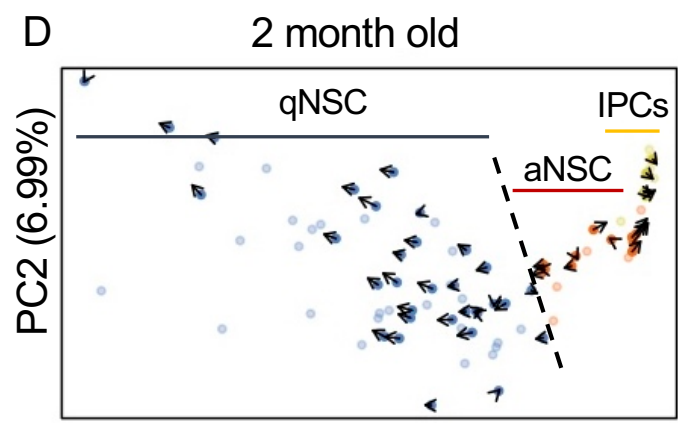

PC1 (71.87\%)

G

qNSC DE
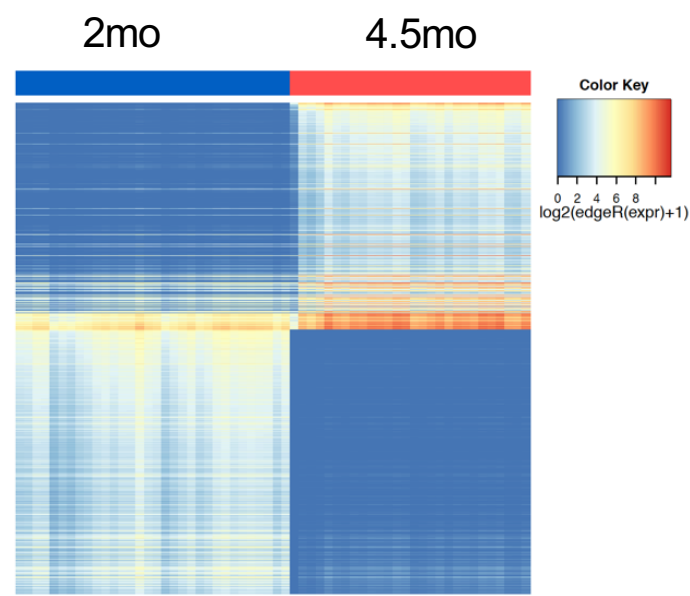

$E$

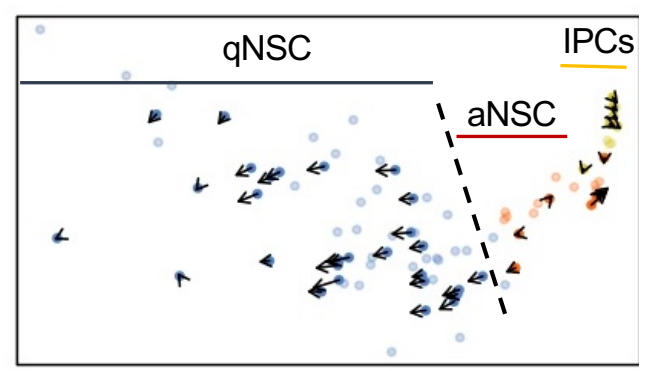

$\mathrm{H}$

Enriched Go term $\quad P$-value $(\square<0.05$; $\square<0.001)$

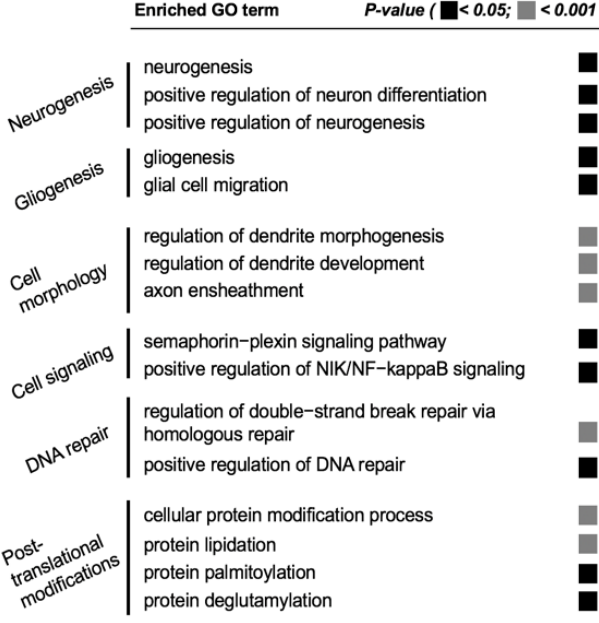

$J$

Phagatr4 şcai

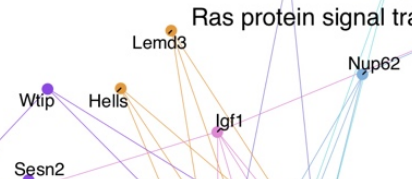

Itga3

(Abl2)

posttranscriptional regulation of gene expression

negative regulation of signaling

Grm5 regulation of gene expression

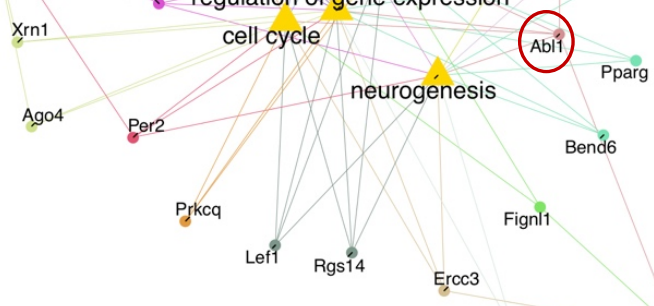

Kdm2a DNA repair

PC1 $(71.87 \%)$
Upregulated

K

10 month old WT

F State changes

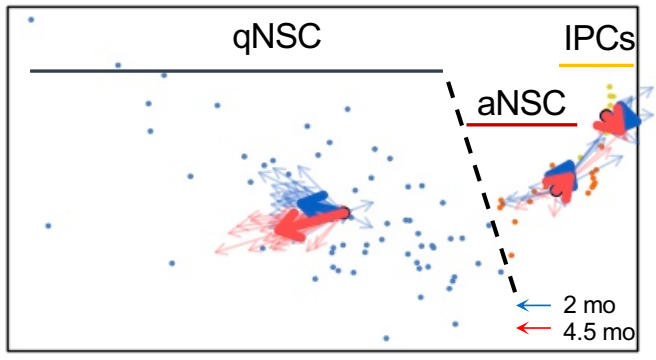

Downregulated

\begin{tabular}{|c|c|}
\hline & Enriched GO term \\
\hline & $\begin{array}{l}\text { neurogenesis } \\
\text { generation of neurons } \\
\text { cell differentiation }\end{array}$ \\
\hline cell cycle & $\begin{array}{l}\text { cell cycle } \\
\text { mitotic cell cycle } \\
\text { mitotic nuclear division }\end{array}$ \\
\hline naling & $\begin{array}{l}\text { negative regulation of signaling } \\
\text { Ras protein signal transduction } \\
\text { regulation of Rho protein signal transduction }\end{array}$ \\
\hline onarepair & $\begin{array}{l}\text { DNA repair } \\
\text { DNA recombination } \\
\text { regulation of DNA metabolic process }\end{array}$ \\
\hline Transcription & $\begin{array}{l}\text { regulation of gene expression } \\
\text { nucleic acid-templated transcription } \\
\text { regulation of transcription, DNA-templated }\end{array}$ \\
\hline Epigenelics & $\begin{array}{l}\text { histone demethylation } \\
\text { posttranscriptional regulation of gene } \\
\text { expression } \\
\text { protein dealkylation }\end{array}$ \\
\hline
\end{tabular}

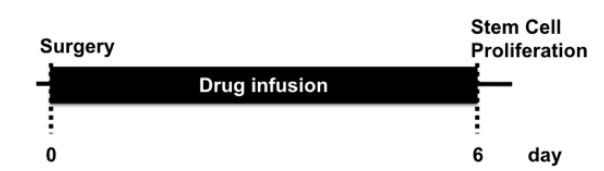

L

M
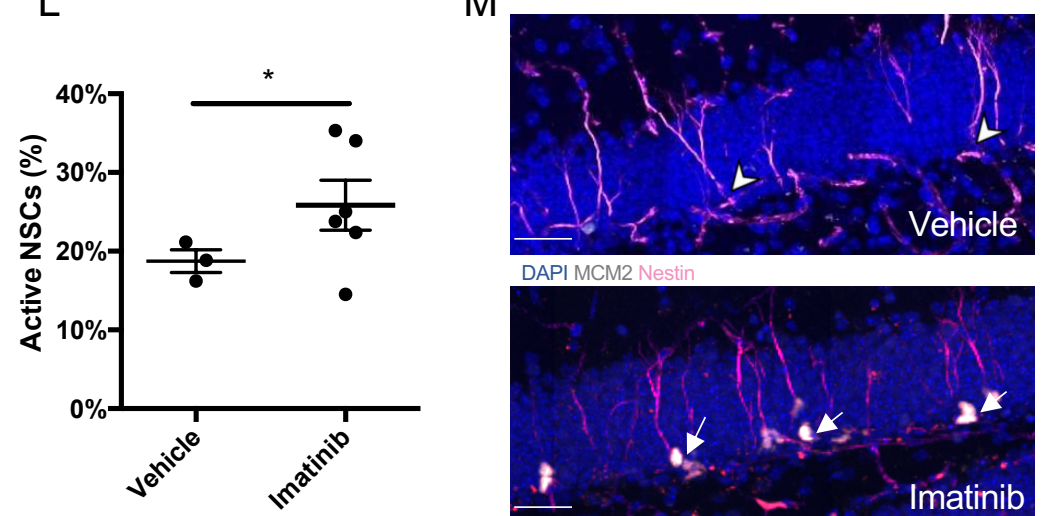
Figure 3. Quiescent NSC aging in the mature hippocampus

(A) Schematic illustration of the single cell RNA sequencing approach.

(B) Principle component (PC) analysis of NSC transcriptomes from 2- and 4.5-month-old mice.

(C) NSCs identified by gene enrichment analysis using Nestin ${ }^{+}$, Vimentin $^{+}, \mathrm{Fabp}^{+}$, Aldoc $^{+}$, Apoe $^{+}$ markers.

(D) RNA velocity defines single cell future states (arrows) identified in quiescent NSCs (qNSC), active NSCs (aNSCs) and IPCs from 2-month-old hippocampus.

(E) RNA velocity defines single cell future states (arrows) identified in qNSCs, active aNSCs and IPCs from 4.5-month-old hippocampus.

(F) RNA velocity analysis reveals NSCs enter a deeper quiescent state in 4.5-month-old mice, whereas some qNSCs from 2-month-old mice will enter cell cycle.

(G) Quiescent NSC differential expression heat map between 2- and 4.5-months old mice.

(H) Top upregulated gene ontology (GO) terms between quiescent NSCs derived from 2-month-old and 4.5-months-old mice. FDR-corrected p-values are shown.

(I) Top downregulated gene ontology (GO) terms between quiescent NSCS derived from 2-month-old and 4.5-months-old mice. FDR-corrected p-values are shown.

(J) String network graph depicting age-related changes downregulated in 4.5-month-old qNSCs. Shown genes have 3+ connections to biological processes.

(K) Schematic illustration of the experimental paradigm and timeline for intracranial drug infusion of Imatinib or vehicle control.

(L) Quantification of the percentage of aNSCs (Nestin ${ }^{+} \mathrm{MCM}^{+}$) among total NSCs in the dentate gyrus of 10-month-old mice infused with vehicle or Imatinib for 6 days. Values represent mean \pm SEM. $N=3$ batches (vehicle), $N=6$ mice (Imatinib -treated). ${ }^{*} p<0.05$, Student's t-test;

(M) Sample confocal images showing immunofluorescence for NSCs $\left(\mathrm{Nestin}^{+}\right)$and cell proliferation $\left(\mathrm{Mcm}^{+}\right)$in the dentate gyrus of 10-month-old mice infused with vehicle or Imatinib. Scale bar, $20 \mu \mathrm{m}$ 


\section{Figure 4}

A
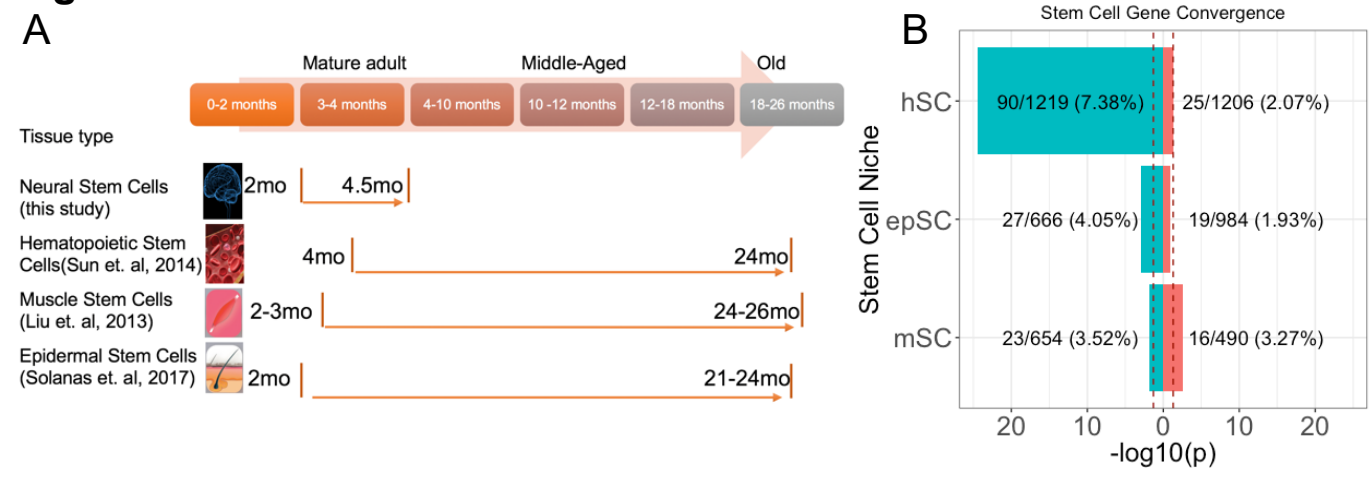

D

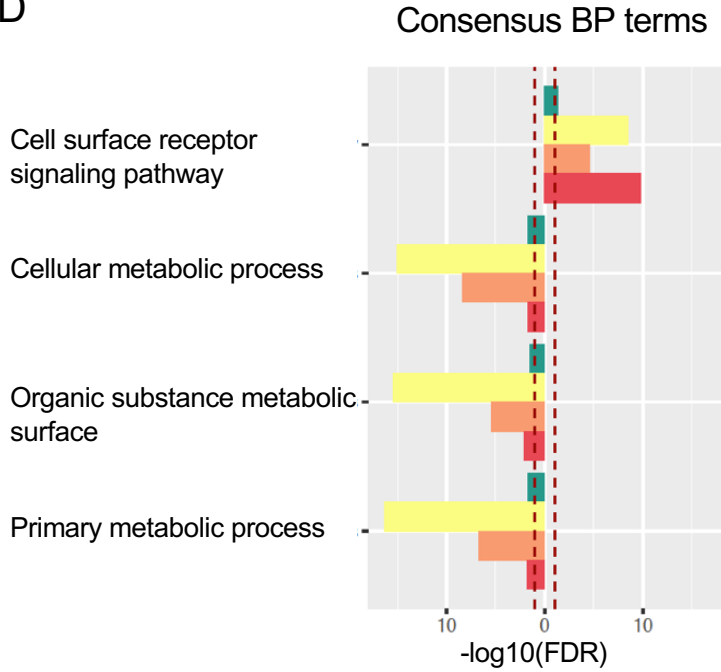

G

\begin{tabular}{l|} 
Metabolism \\
RNA \\
homeostasis \\
Protein \\
homeostasis
\end{tabular}

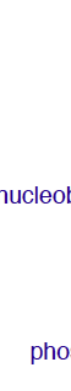
phosphorylation -
regulation of transforming growth factor beta receptor signaling pathway -
canonical Wnt signaling pathway negative regulation of extrinsic apoptotic signaling pathway regulation of transmembrane receptor protein serine/threonine kinase signaling pathway cytokine-mediated signaling pathway cell surface receptor signaling pathway involved in cell-cell signaling -
$\mathrm{H}$

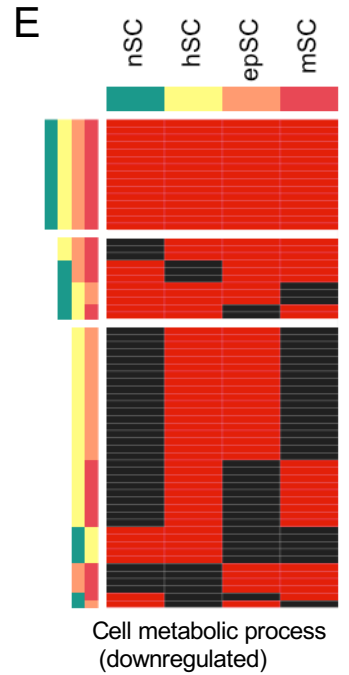

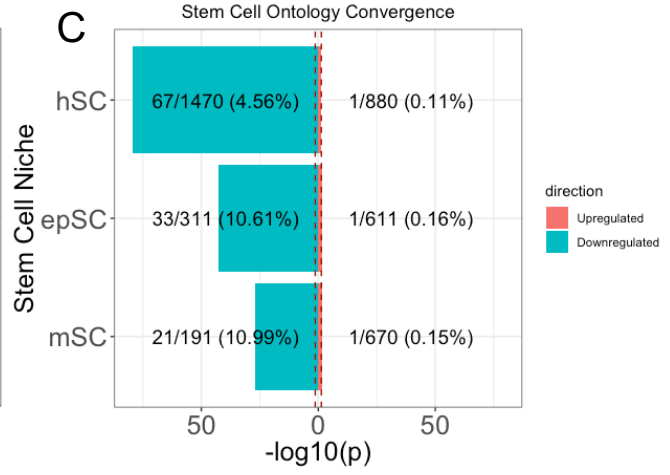

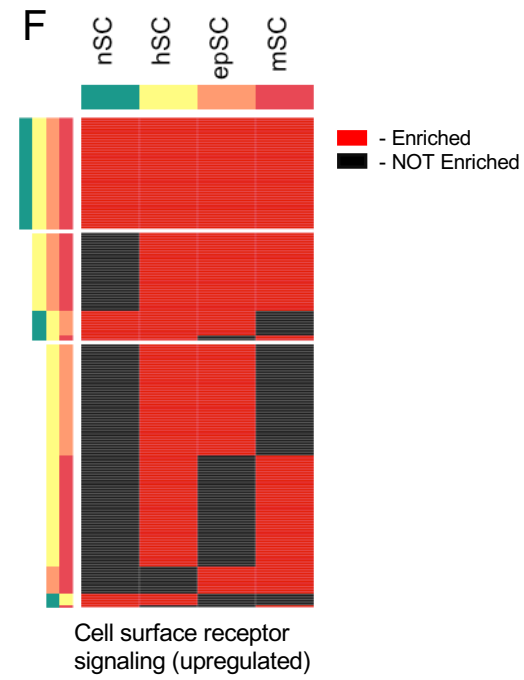

\section{Consensus Offspring terms}

cellular macromolecule metabolic process cellular catabolic process nucleic acid metabolic process RNA metabolic process RNA biosynthetic process transcription, DNA-templated transcription, DNA-templated -
RNA polymerase II promoter cellular protein metabolic process regulation of cellular protein metabolic process hosphate-containing compound metabolic process peptidyl-amino acid modification -

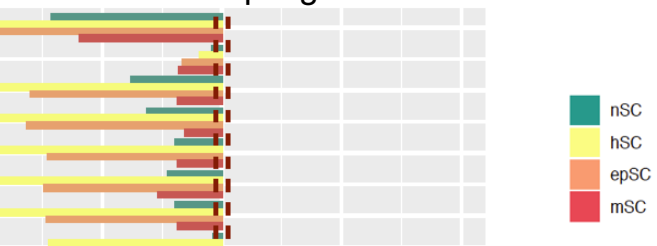

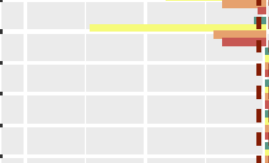

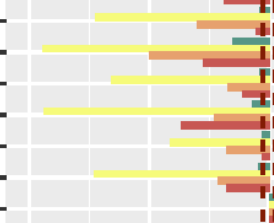


Figure 4. Consensus signatures of stem cell aging

(A) Experimental approach to compare mature NSC with old hematopoietic (hSC), muscle (mSC) and epidermal (eSC) stem cell transcriptomes.

(B) Pairwise stem cell gene convergence between NSCs and hSCs, mSCs, eSCs. FDR-corrected p-values are shown.

(C) Pairwise stem cell ontology convergence between NSCs and hSCs, mSCs, eSCs. FDRcorrected p-values are shown;

(D) Consensus biological processes shared across all stem cell niches. FDR-corrected p-values are shown.

(E) Cell metabolic processes shared by various stem cell compartments.

(F) Cell surface receptor signaling shared by various stem cell compartments.

(G) Top consensus up- and downregulated offspring terms shared across four stem cell niches. Metabolism, RNA homeostasis and proteostasis are downregulated. Regulation of Wnt, TGF $\beta$ and apoptotic signaling is upregulated. FDR-corrected p-values are shown.

$(\mathrm{H})$ String network analysis showing unique or shared upregulated genes associated with negative regulation of apoptotic signaling pathway in NSCs, hSCs, mSCs, and eSCs.

(I) String network analysis showing unique or shared downregulated genes associated with transcription from RNA polymerase II promoter in in NSCs, hSCs, mSCs, and eSCs. 


\section{A}

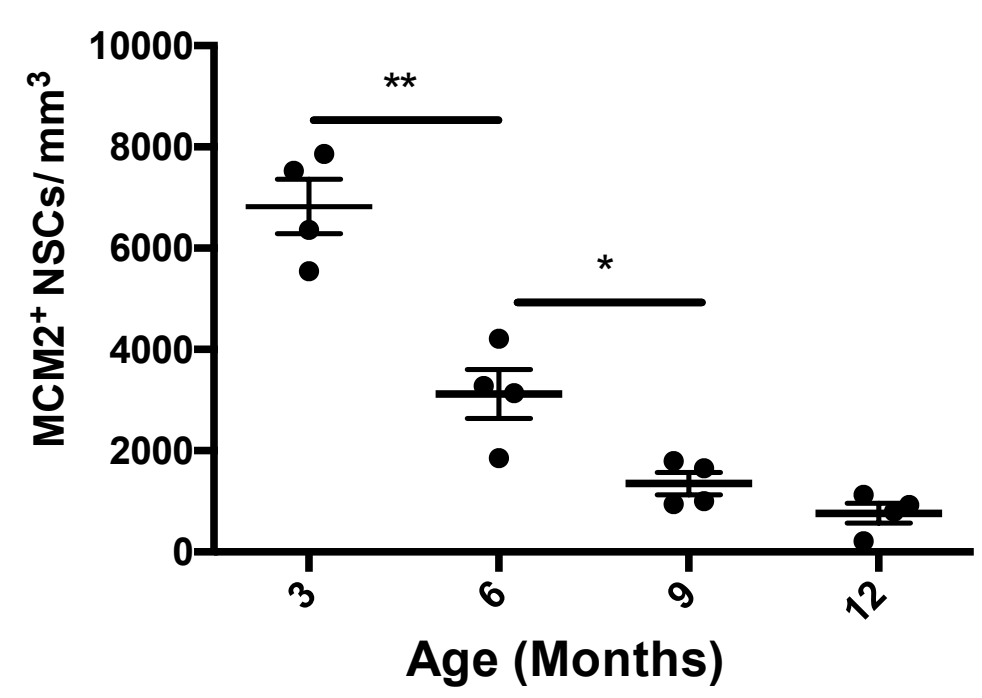

B

\begin{tabular}{|c|c|c|c|c|c|c|c|c|}
\hline \multirow[b]{2}{*}{ Age of mice (mo) } & \multicolumn{8}{|c|}{ Nestin" } \\
\hline & 2 & & 3 & 4 & 6 & & & 8 \\
\hline Tracing duration (dpi) & 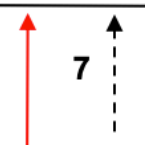 & 30 & 60 & 120 & $\mid 7$ & 30 & 60 & \\
\hline
\end{tabular}

C

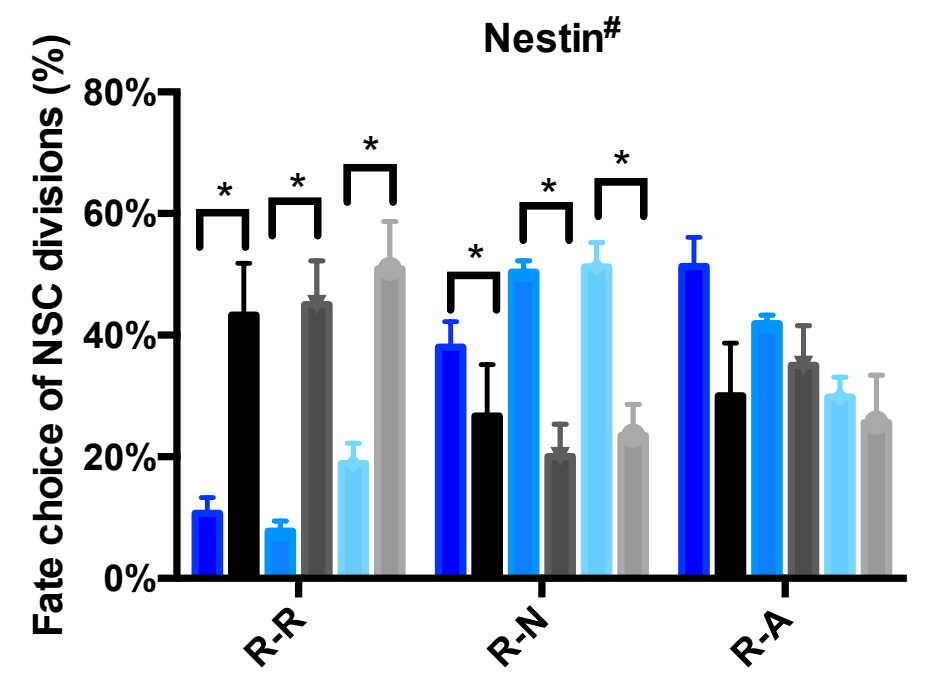

D

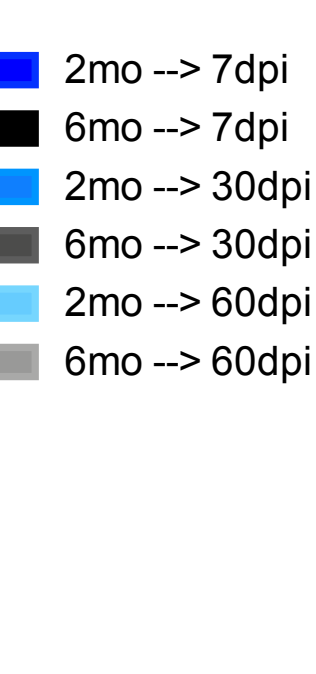

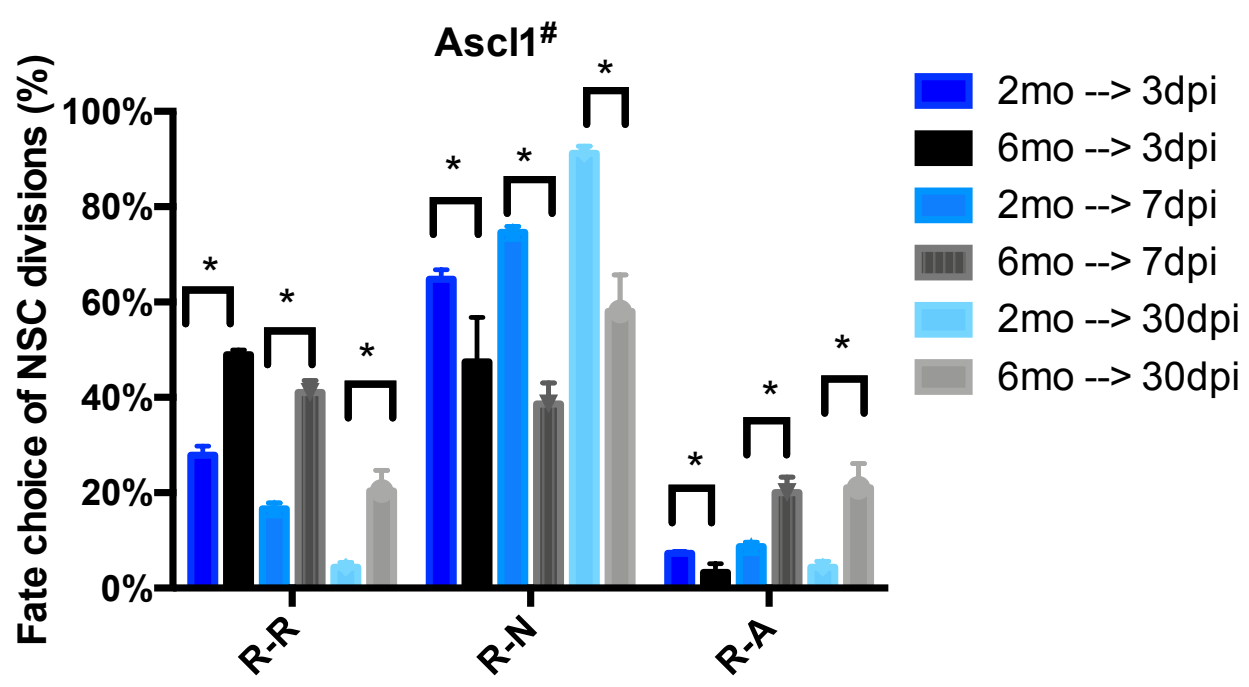


Figure S1. Population analysis and clonal lineage-tracing of individual NSCs in the adult mouse dentate gyrus, related to Figure 1.

(A) Quantification of the total number of active NSCs in the dentate gyrus across ages. $N=4-6$ mice; Values represent mean \pm SEM. ${ }^{*} p<0.05,{ }^{* *} p<0.01$; Student's t-test.

(B) Experimental design of single cell linage tracing for Nestin\#-NSC and Ascl1\#-NSC subpopulations, results in Table S4.

(C) Quantification of cell fate choice outcome for 2 and 6 months old Nestin\#-NSC clonal tracing 7, 30 and 60 days after labeling. $R=$ radial glia-like neural stem cells; $A=A$ stroglial lineage; $N=$ neuronal lineage. Values represent mean \pm SEM ( $N=20-101$ clones, see details in Table S4; ${ }^{*} p<0.05$, ${ }^{* *} p<0.01$; Student's t-test $)$;

(D) Quantification of cell fate choice outcome for 2 and 6 months old Ascl1\#-NSC clonal tracing 3, 7 and 30 days after labeling. $R=$ radial glia-like neural stem cells; $A=A$ stroglial lineage; $N=$ neuronal lineage. Values represent mean \pm SEM $\left(N=20\right.$-101 clones, see details in Table S4; ${ }^{*} p<0.05,{ }^{* *} p<0.01$; Student's t-test). 

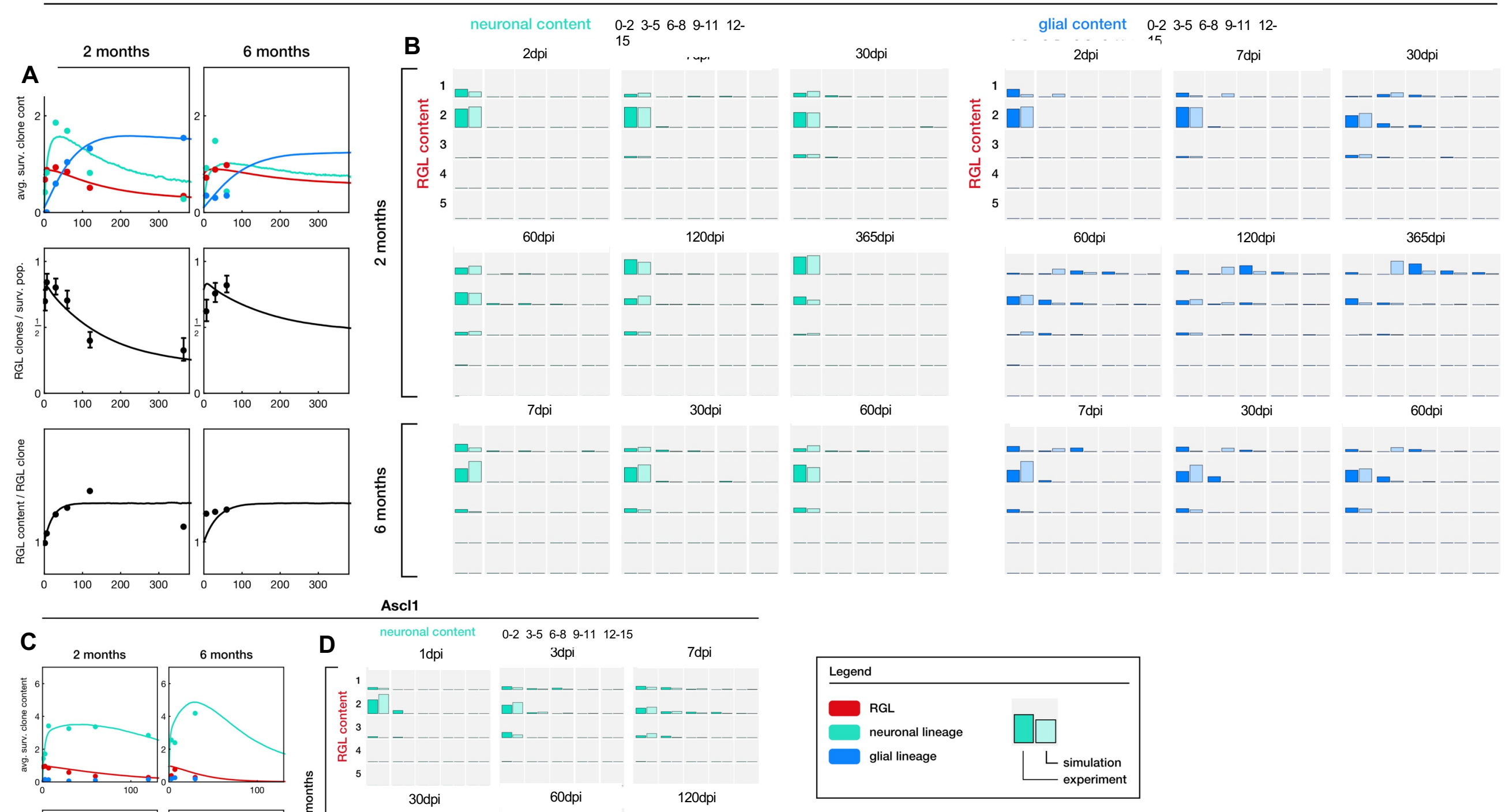

Ascl1
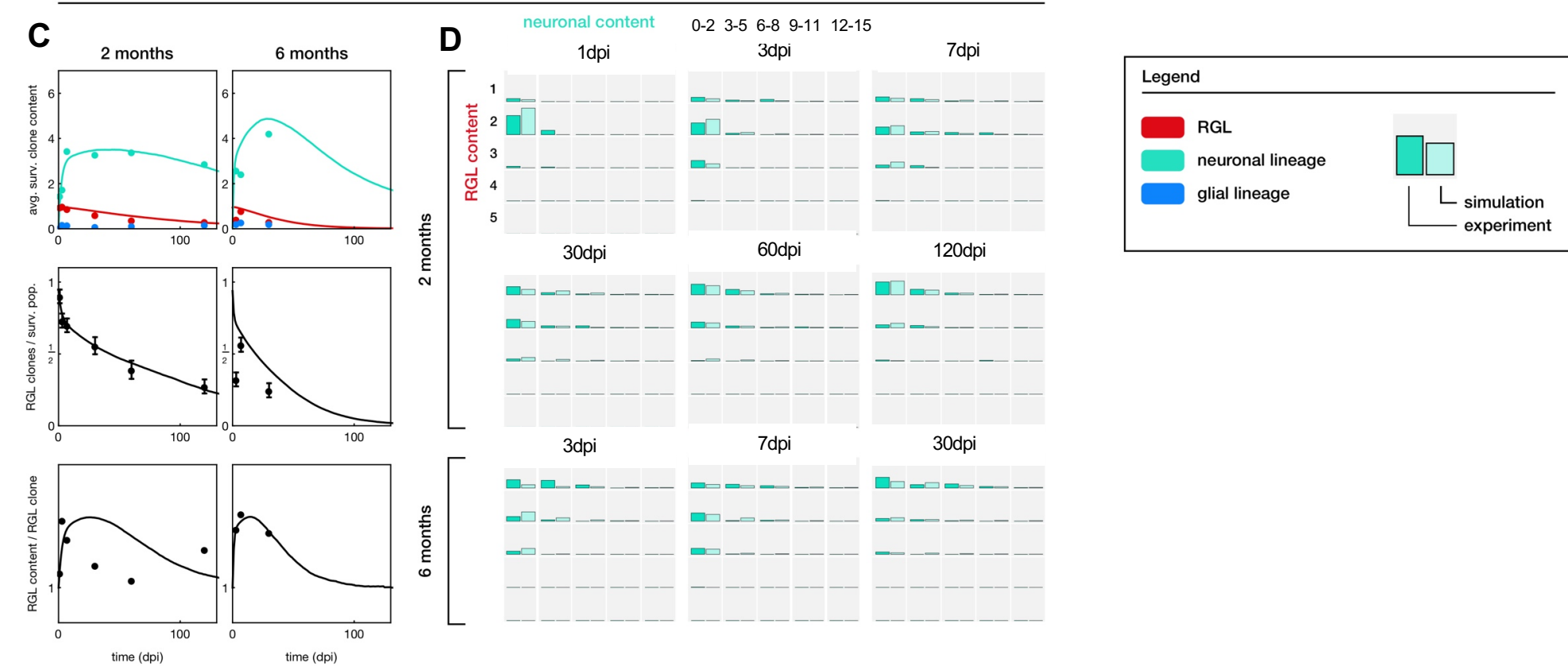
Figure S2. Computational modeling of Nestin\#-NSC and Ascl1\#-NSC, related to Figures 1 and 2.

(A) Model of stochastic Nestin\#-NSC fate dynamics within a small stem cell niche. NSC clonal content and survival increases in 6 month-old mice, while neuronal and astroglial content decreases compared to 2 month-old mice. Red=radial glia-like NSC content, blue=astroglial content, green=neuronal content; Line=model, dots=experimental data.

(B) Least-fit squares showing correspondence between the simulated model and clonal content from Nestin\#-NSCs traced in 2 months-old and 6 months-old mice.

(C) Model of a developmental-like stem cell fate program in Ascl1\#-NSCs. NSC clonal survival decreases in 6 month-old mice, while neuronal content increases compared to 2 month-old mice. Red=radial glia-like NSC content, blue=astroglial content, green=neuronal content.

(D) Least-fit squares showing correspondence between the simulated model and clonal content from Ascl1 ${ }^{\#-N S C s}$ traced in 2 months-old and 6 months-old mice. 


\section{Figure S3}

A

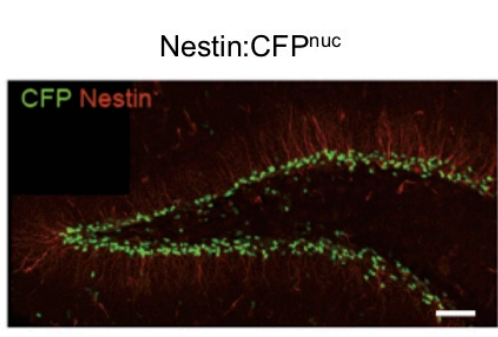

Dentate gyrus microdissection Single cell dissociation

B
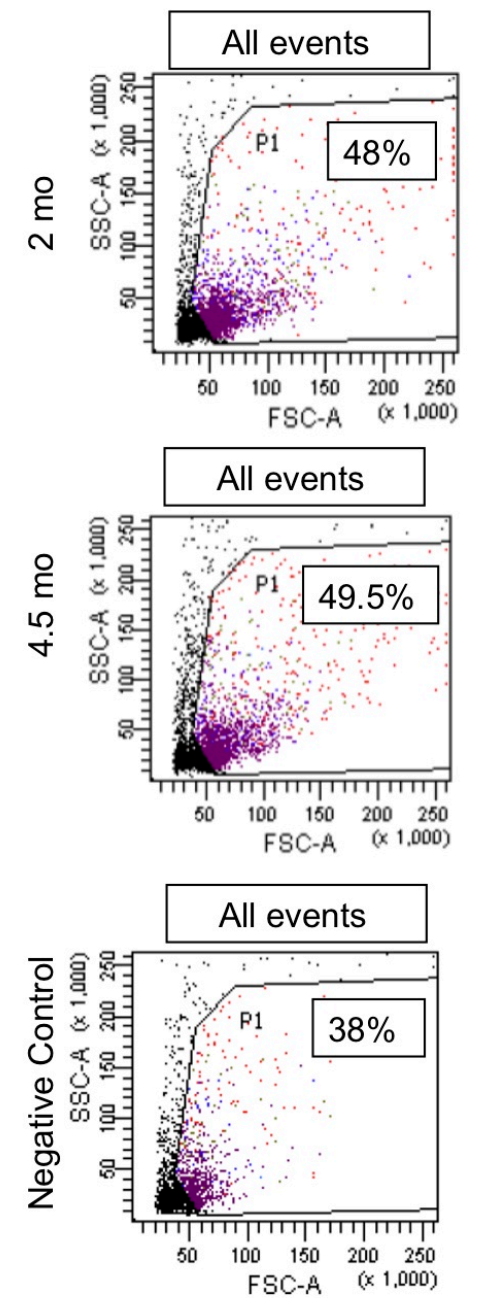

Single cell collected

into the $1.75 \mathrm{uL}$ of

Lysis Mixture

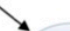

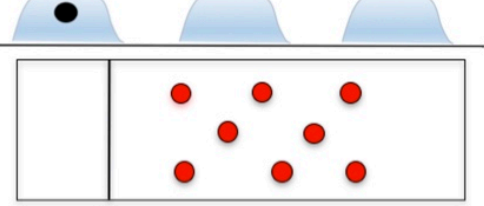

FACs sorting on the glass slide

Nextera XT library preparation Sequencing

\section{SMARTSeq4 + Illumina}

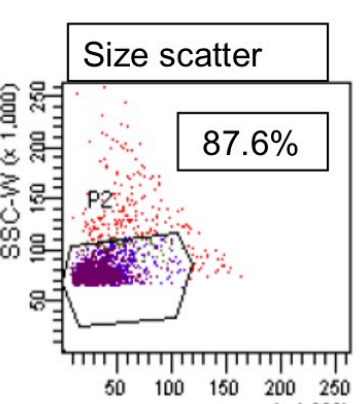

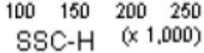

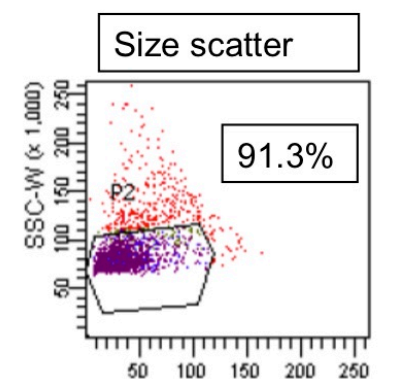

SSC-H $\quad(x 1,000)$

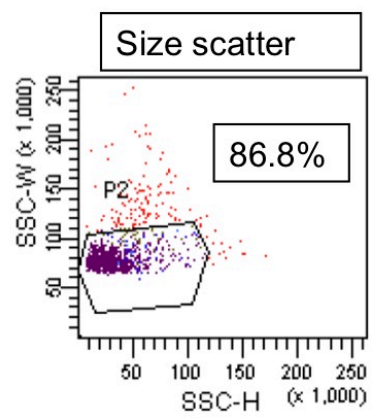

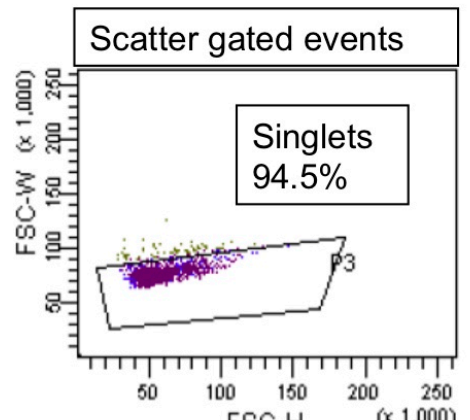
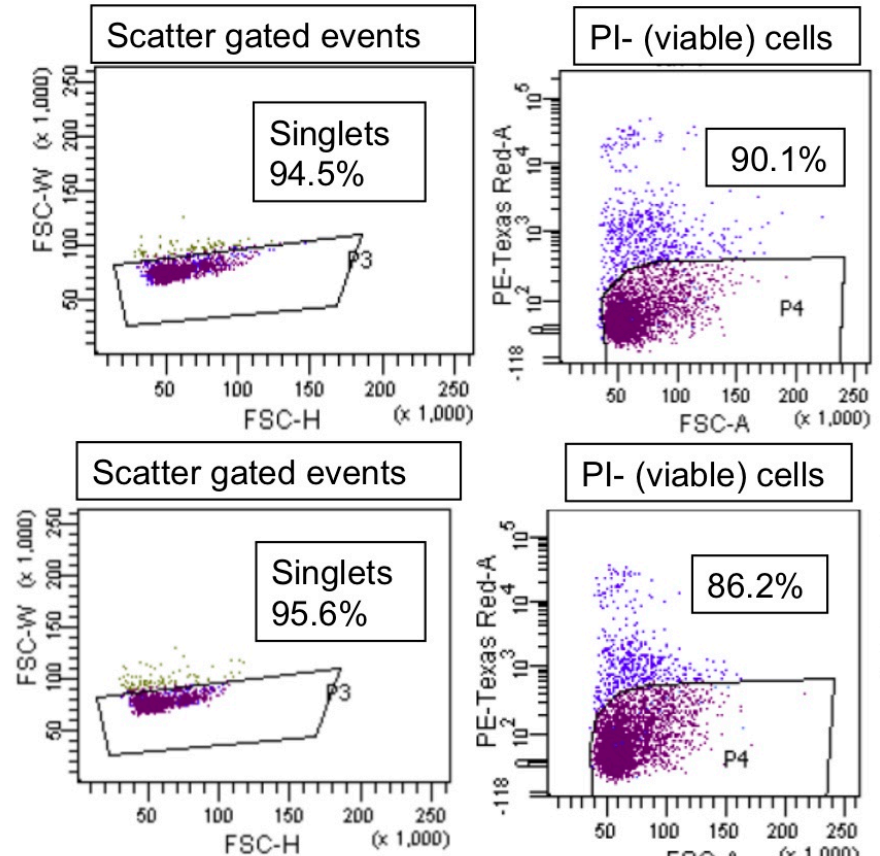

$\begin{array}{lllll}50 & 100 & 150 & 200 & 250\end{array}$
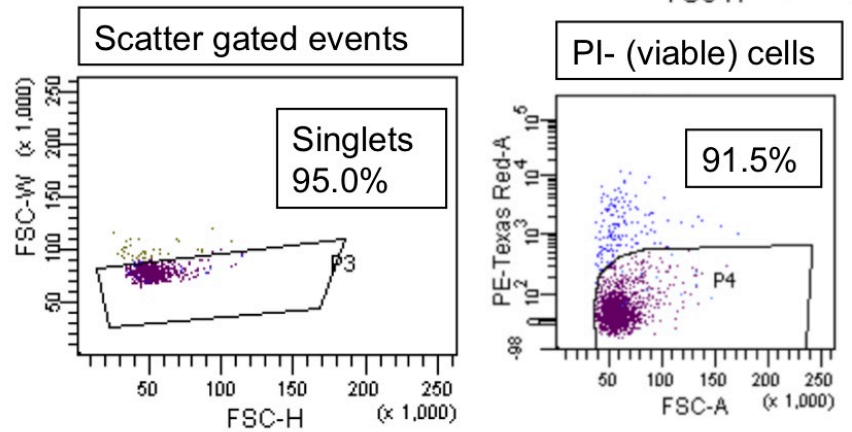

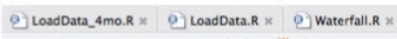

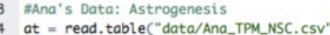

Fremove outlier N_GP.15

Bioinformatic analysis
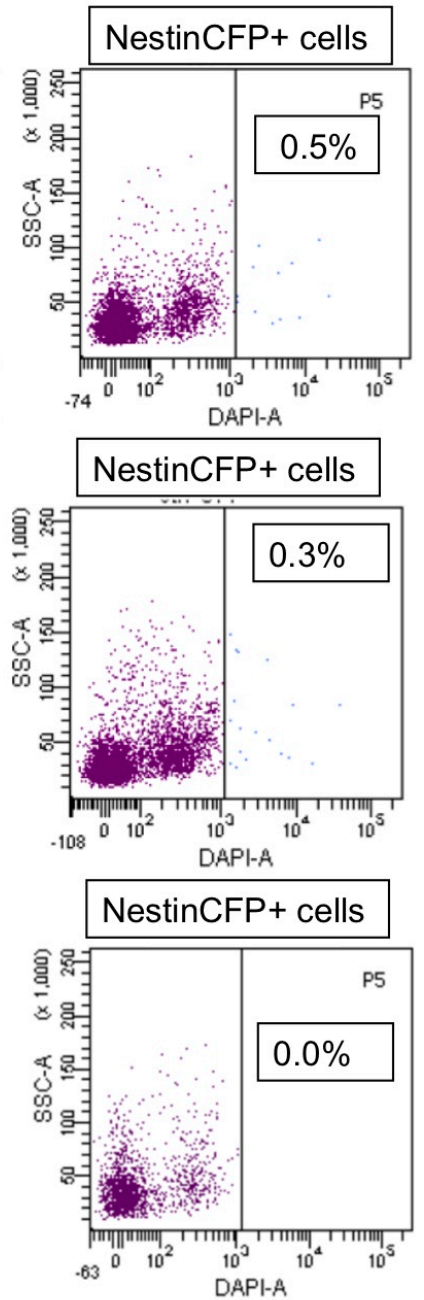
Figure S3. Experimental design of scRNA-seq experiment, related to Figure 3 and 4.

(A) Experimental steps to generate single cell transcriptomes from 2 months-old and 4.5 months-old Nestin::CFP mice. Scale bar, $50 \mu \mathrm{m}$.

(B) FACS sorting scheme used to isolate cells from the dentate gyrus of 2 months-old and 4.5 months-old Nestin::CFP mice. Negative controls were used as indicated. 


\section{Figure S4}
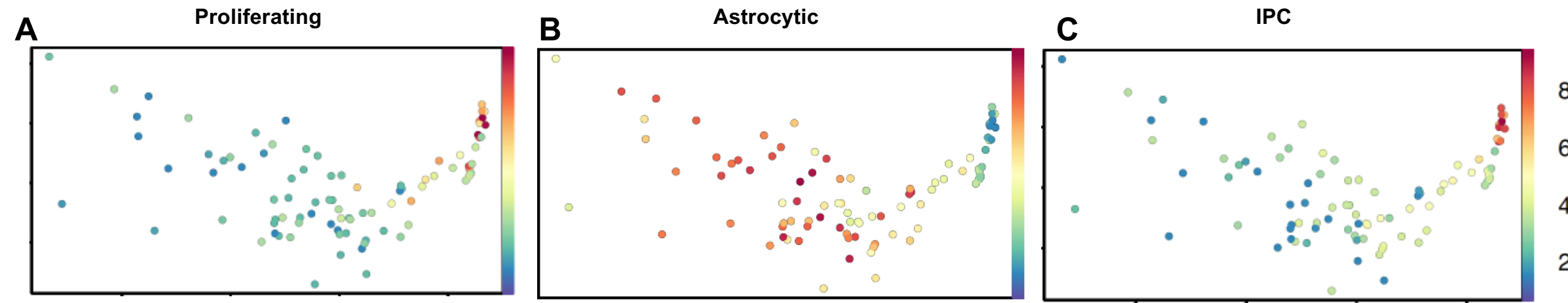

D

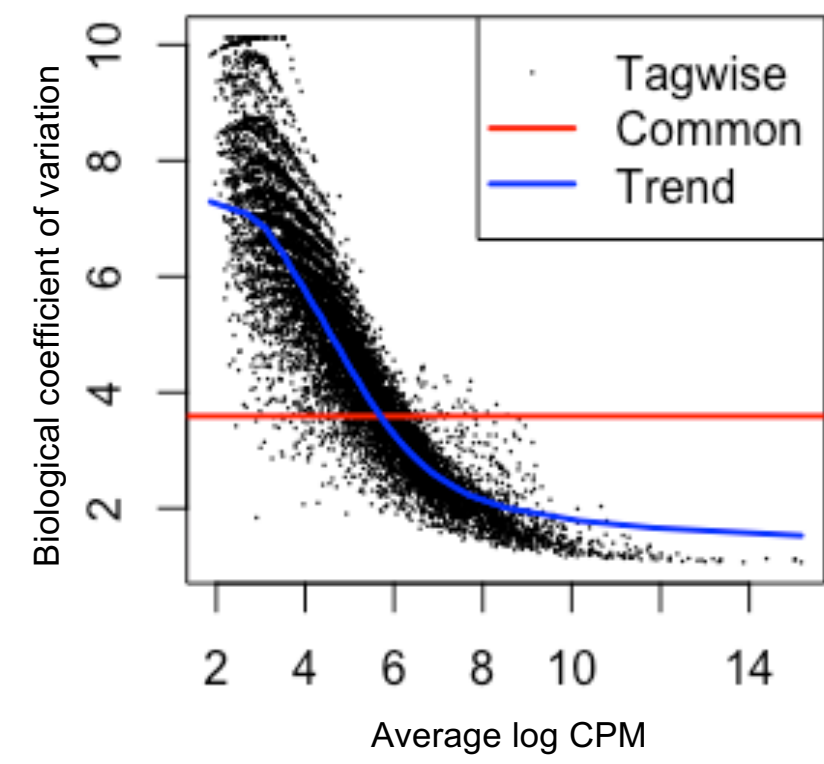

E

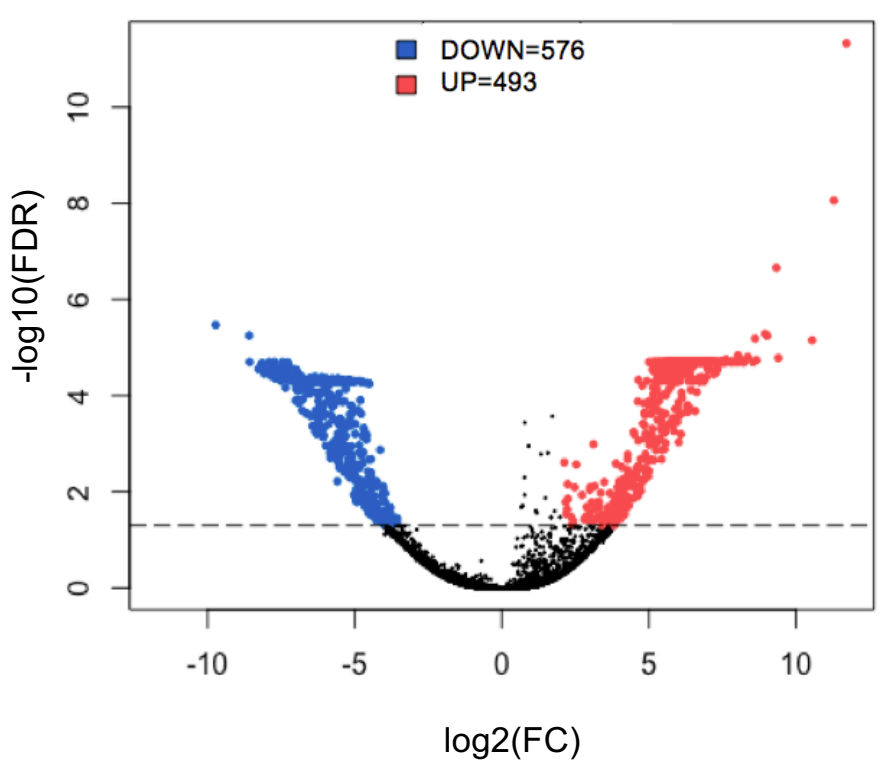

F

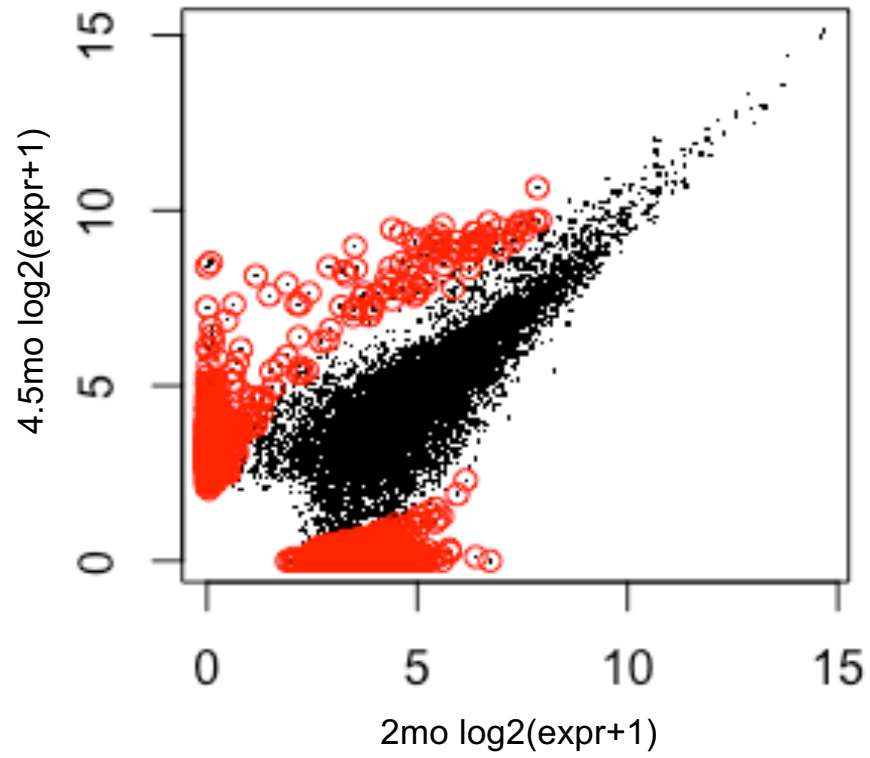


Figure S4. Differential gene expression analysis related to Figure 3 and 4.

(A) Principle component plot highlighting proliferating cells marked by log-mean expression of PCNA, Mcm7, Cdk4, Cdk6.

(B) Principle component plot highlighting astroglial properties marked by log-mean expression $\mathrm{S} 100 \beta$, Aldh111, Megf10, Ntsr2, Acsbg1.

(C) Principle component plot highlighting intermediate progenitor cells marked by log-mean expression Eomes/Tbr2, Sox11, Stmn1.

(D) Biological coefficient of variation between 2 months-old and 4.5 months-old across average log counts per million (CPM). A greater degree of relative variation occurs at lower CPM levels justify a need for edgeR to call differentially expressed genes.

(E) Volcano plot of edgeR-called differentially expressed genes between quiescent NSCs from 2 monthsold and 4.5 months-old mice. FDR-corrected p-values are shown.

(F) Mean-mean gene expression scatter plot between NSCs from 2 months-old and 4.5 months-old mice highlighting differentially expressed genes (red). 


\section{Figure S5}

A

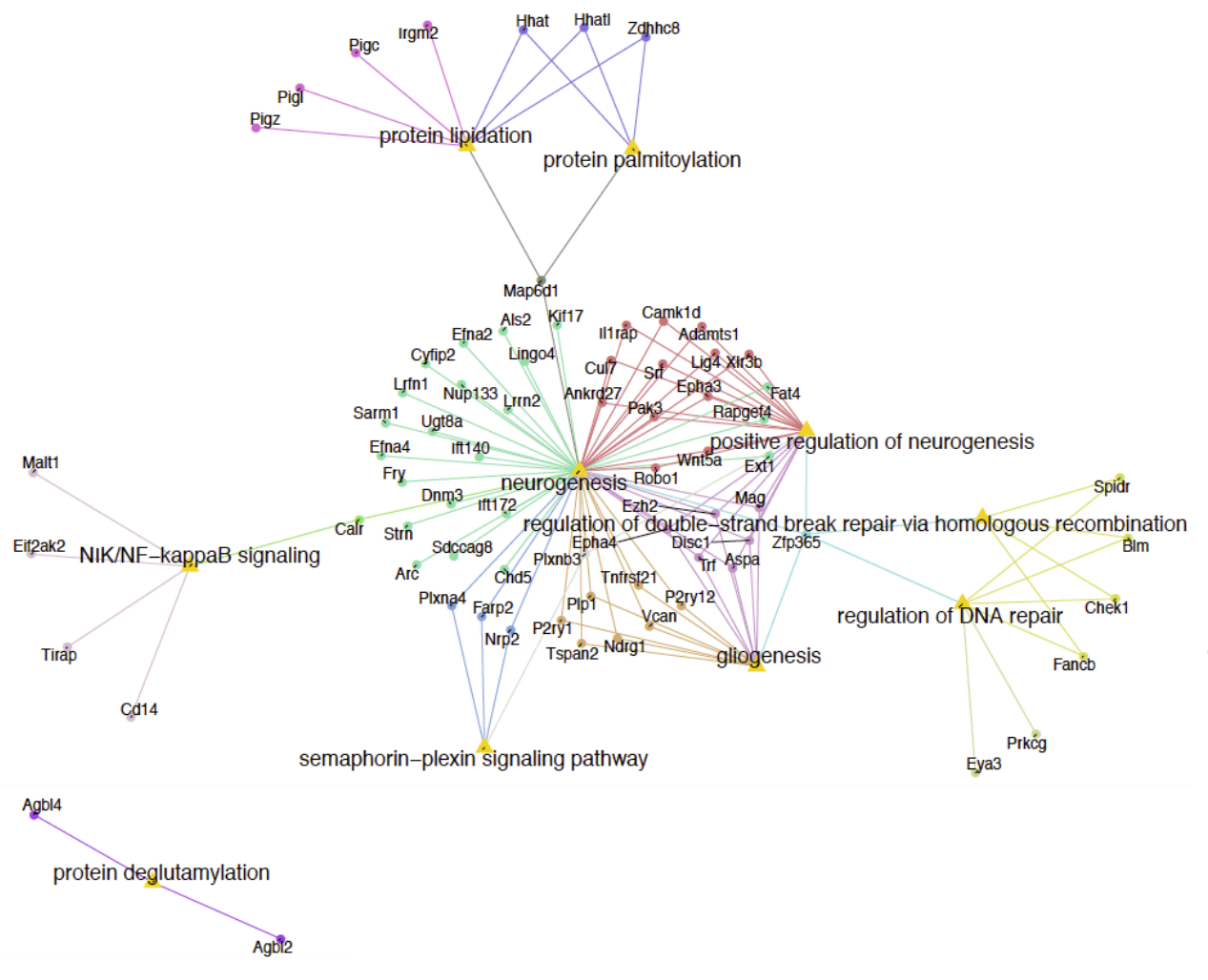

\section{B}

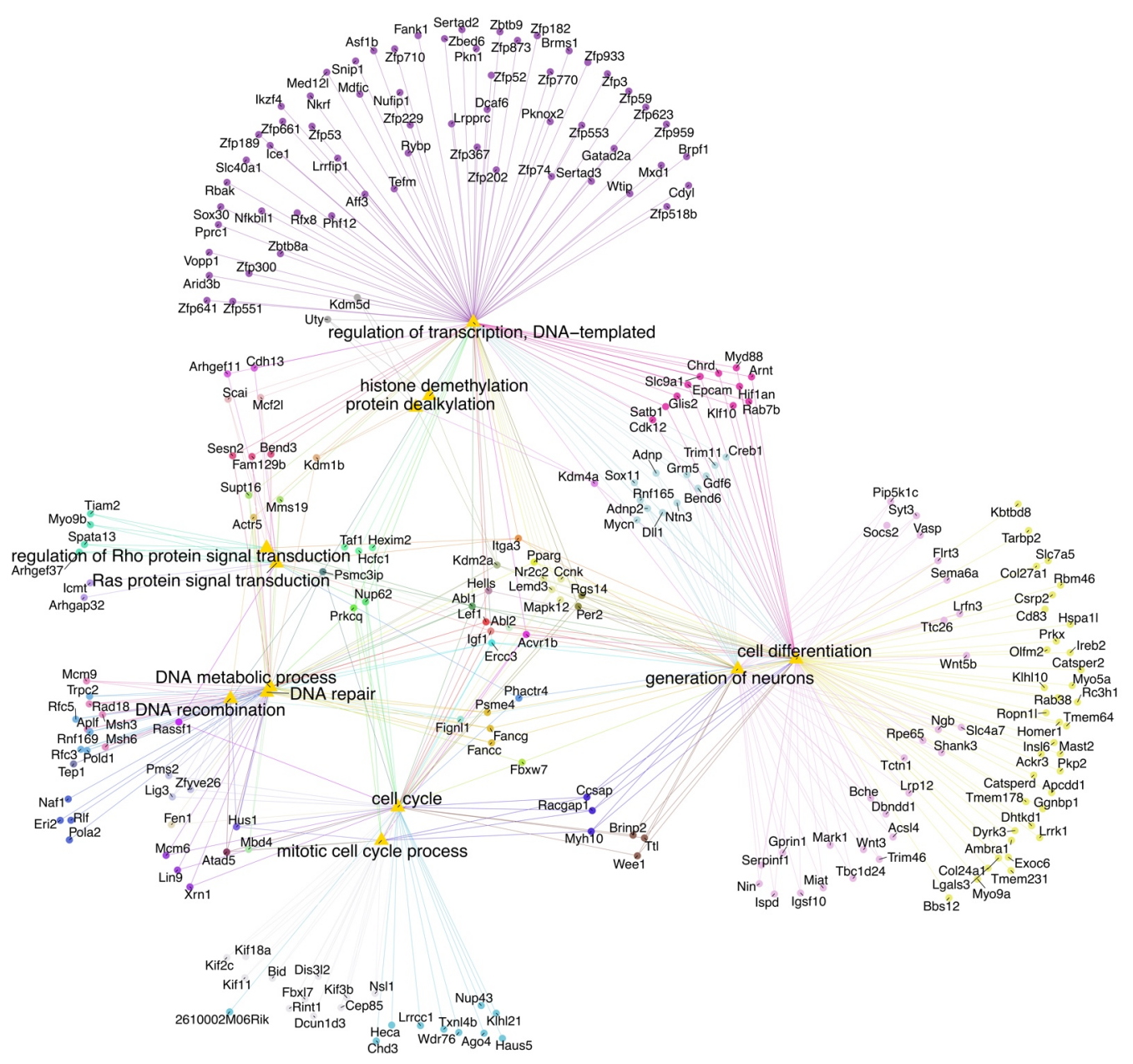


Figure S5. String gene network graphs across up/down regulated GO terms, related to Figure 3.

(A) String network graph depicting age-related changes upregulated in 4.5 month-old vs 2 month-old quiescent NSCs. Shown genes have 1+ connections to biological processes.

(B) String network graph depicting age-related changes downregulated in 4.5 month-old vs 2 monthold quiescent NSCs. Shown genes have 1+ connections to biological processes. 
Figure S6

A

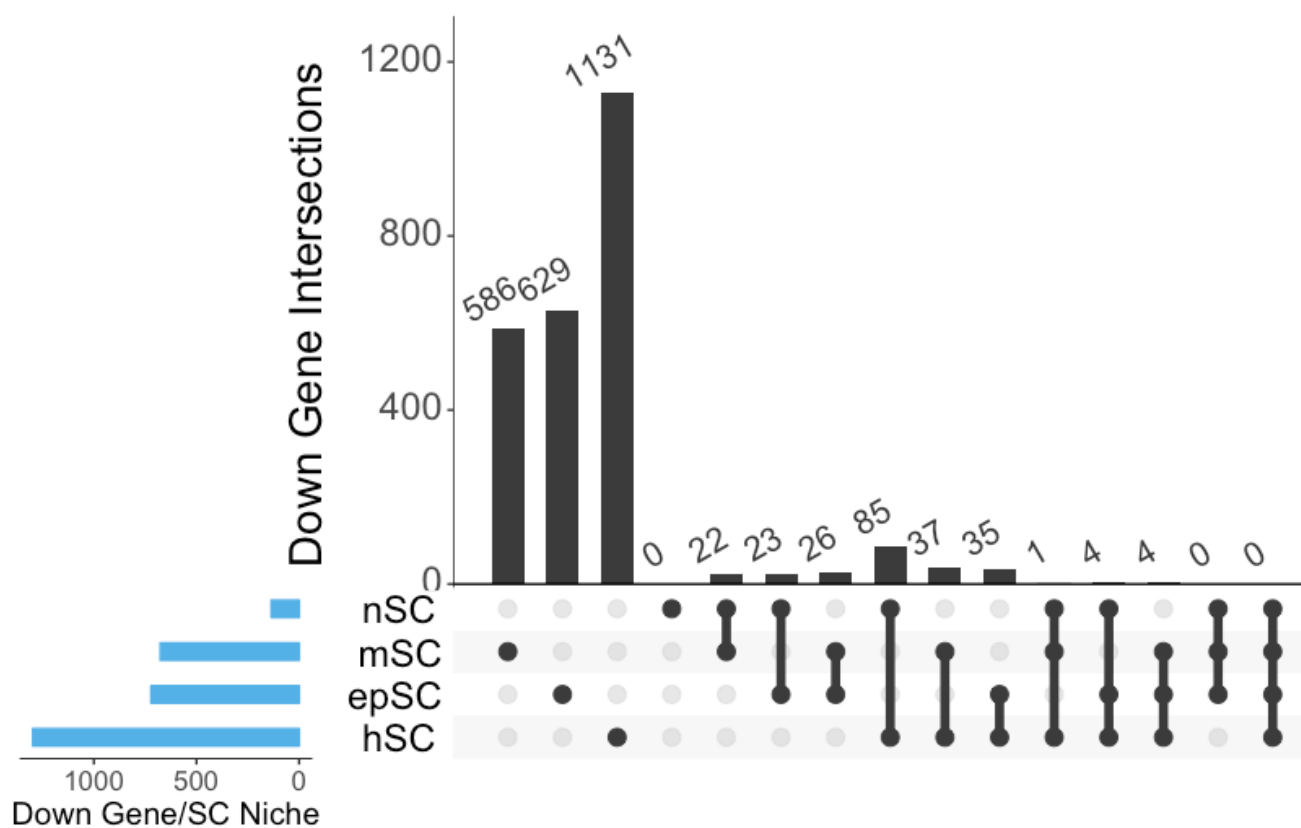

B

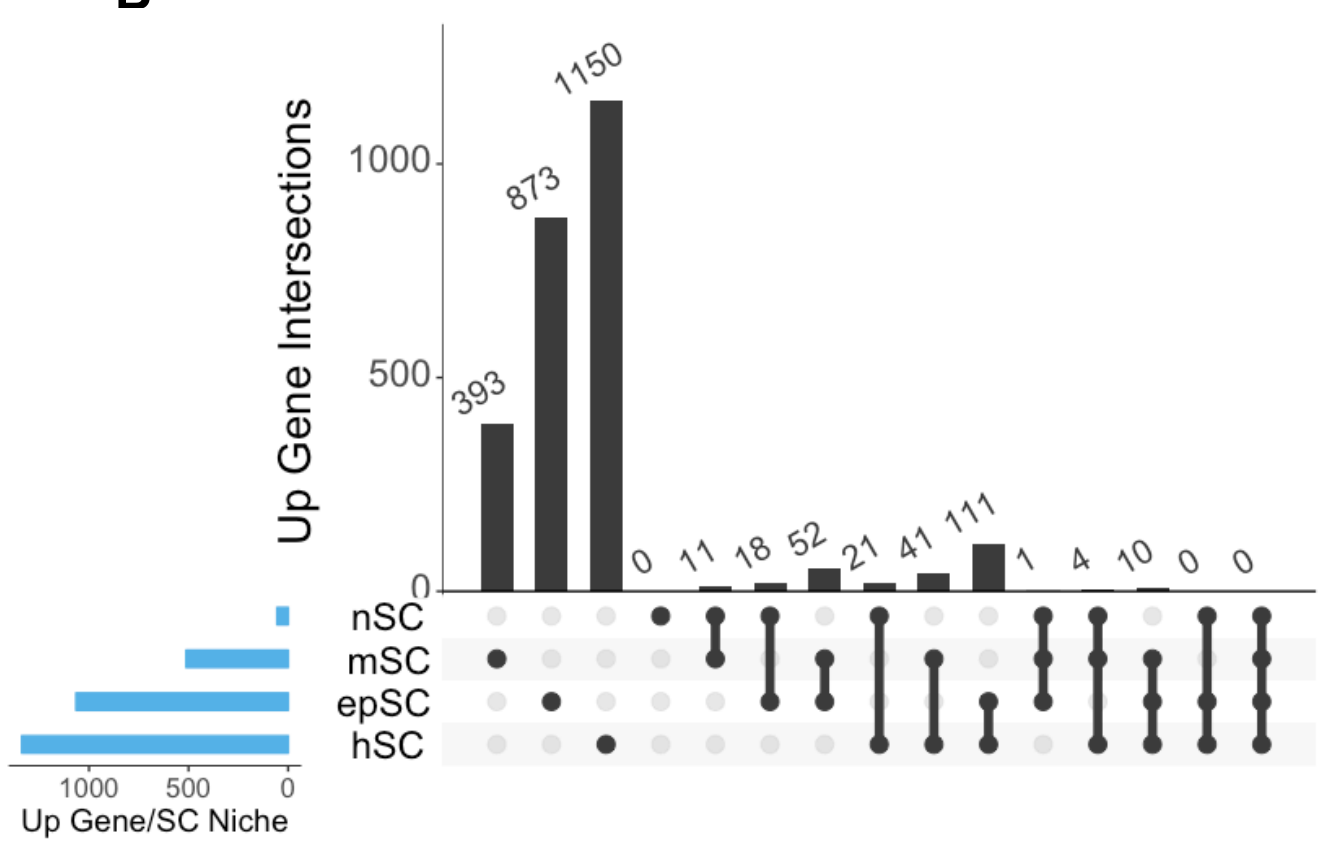

D

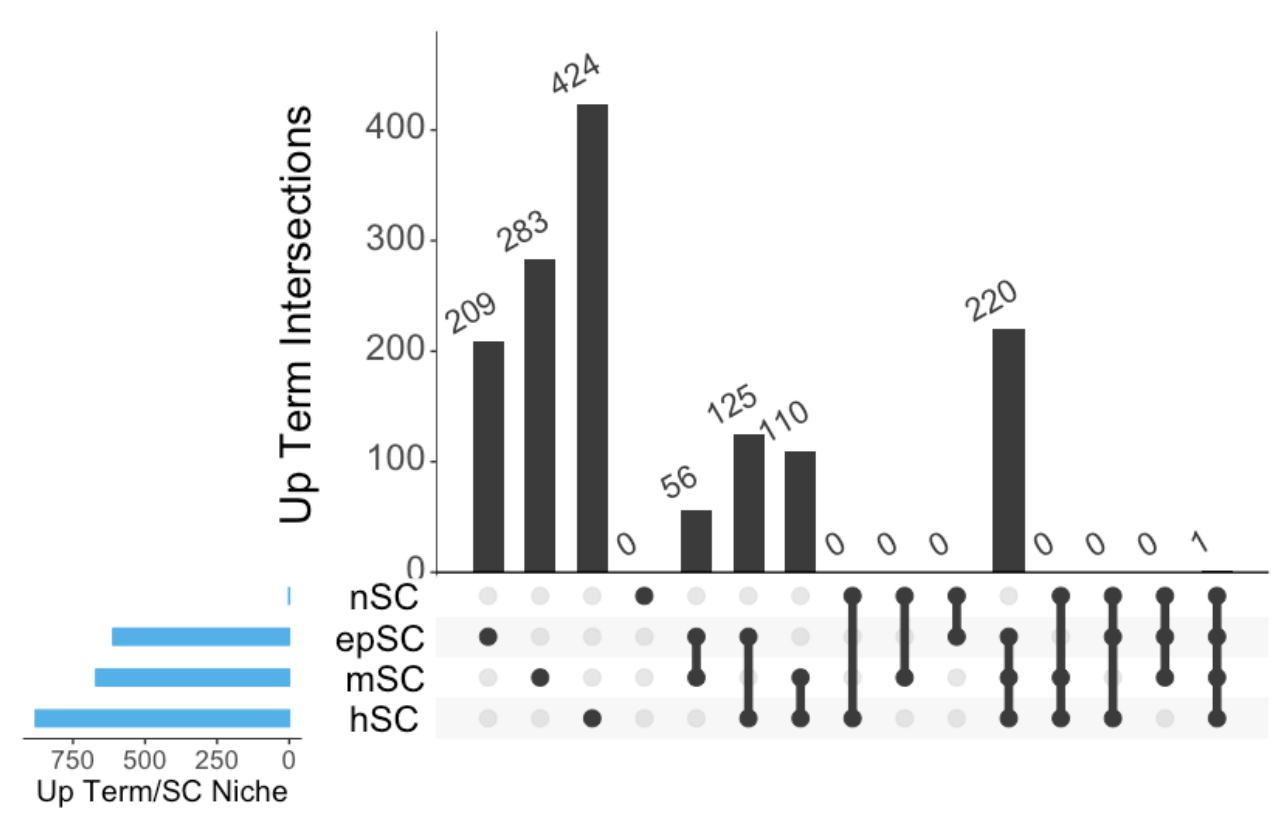

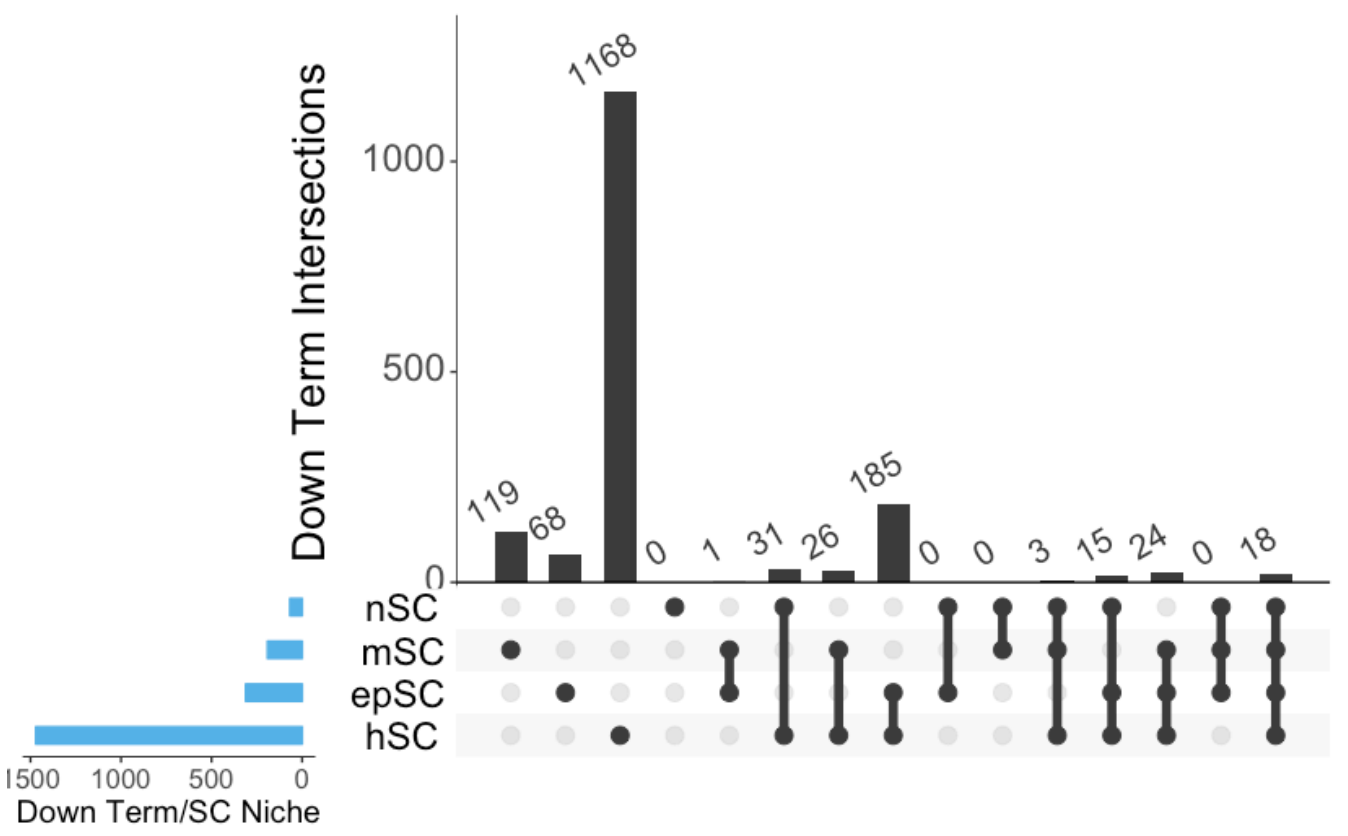


Figure S6. Gene and term intersect assessment across multiple stem cell niches, related to Figure 4.

(A) Upregulated gene intersect plot across NSC, hematopoietic, muscle and epidermal stem cell transcriptomes;

(B) Downregulated gene intersect plot across NSC, hematopoietic, muscle and epidermal stem cell transcriptomes;

(C) Upregulated term intersect plot across NSC, hematopoietic, muscle and epidermal stem cell transcriptomes;

(D) Downregulated term intersect plot across NSC, hematopoietic, muscle and epidermal stem cell transcriptomes. 


\section{Figure $\mathbf{S 7}$}

A Cytokine-mediated signaling pathway

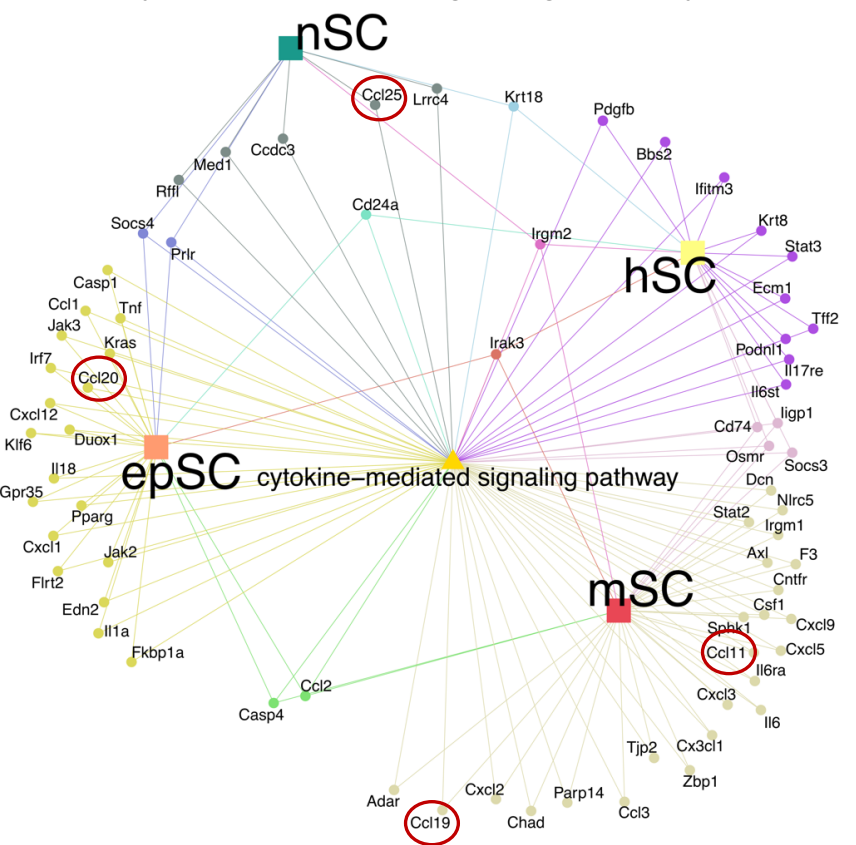

D Cell surface receptor signaling pathway

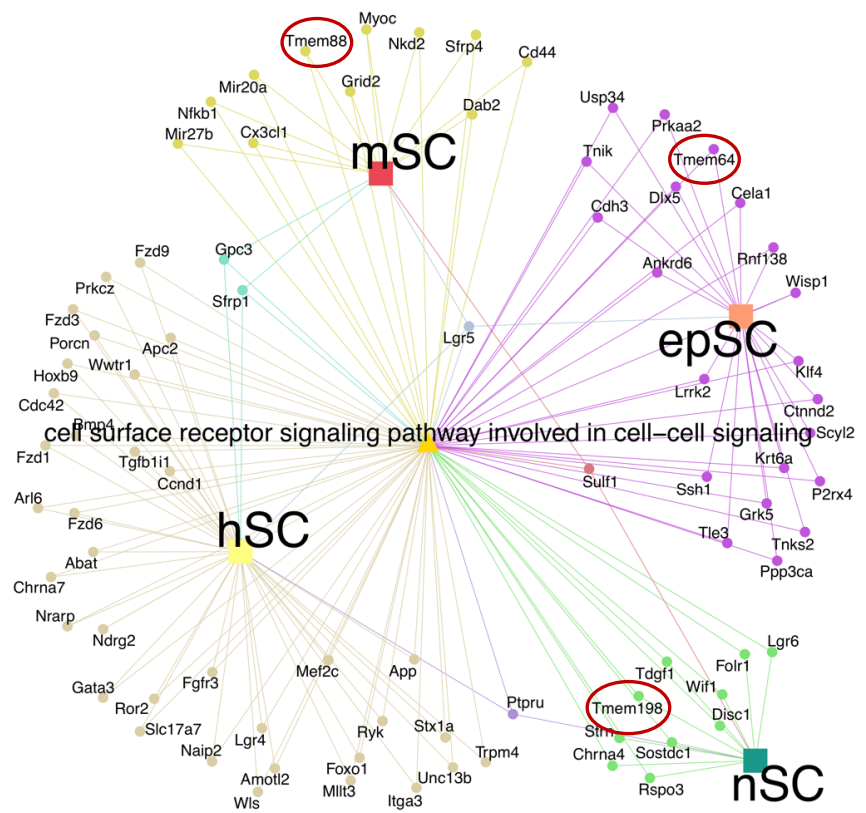

B Regulation of TGF- $\beta$ signaling pathway $\mathrm{nSC}$

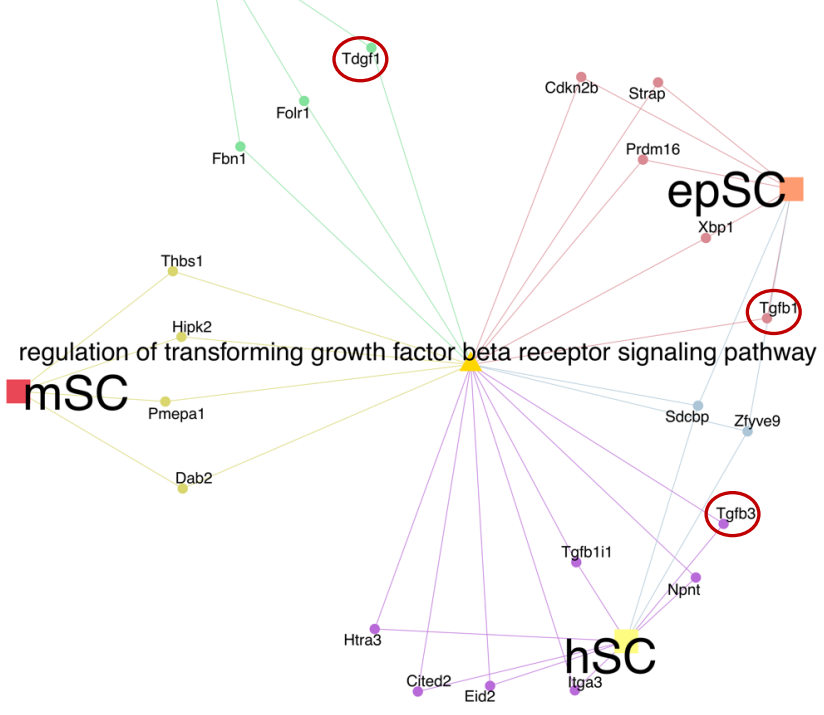

E Transmembrane receptor protein serine/threonine kinase signaling pathway

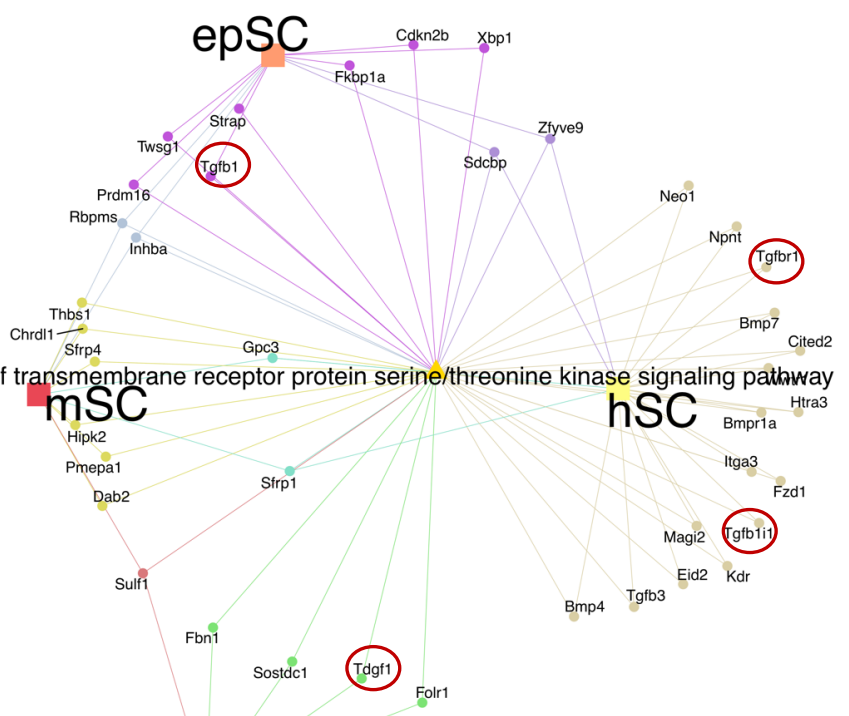

$n S C$
C Negative regulation of apoptotic signaling pathway

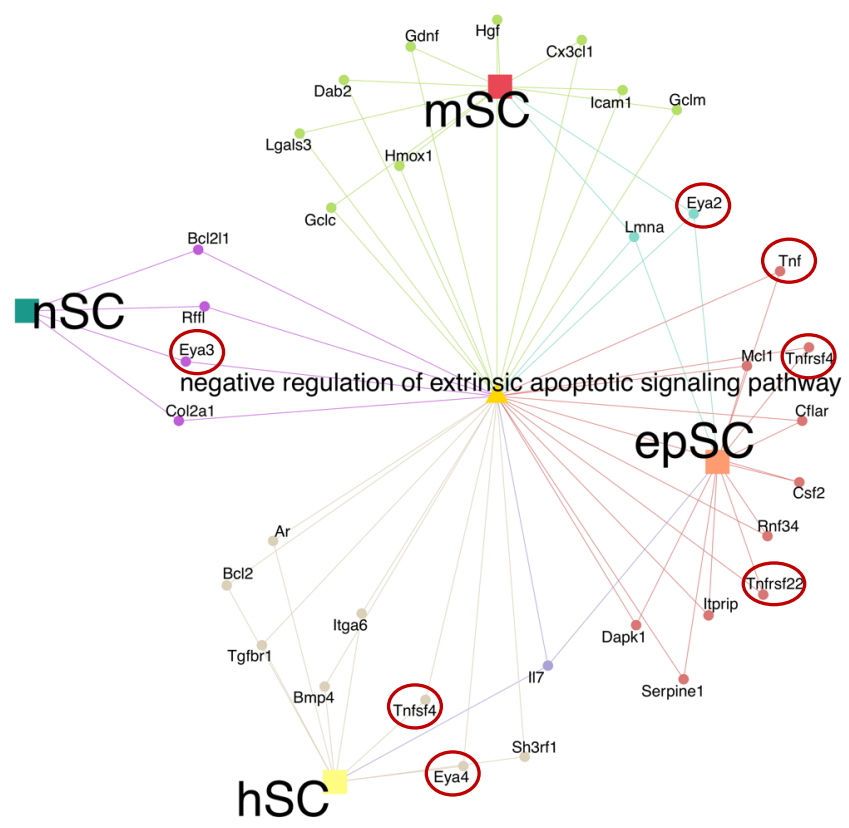

F Canonical Wnt signaling pathway

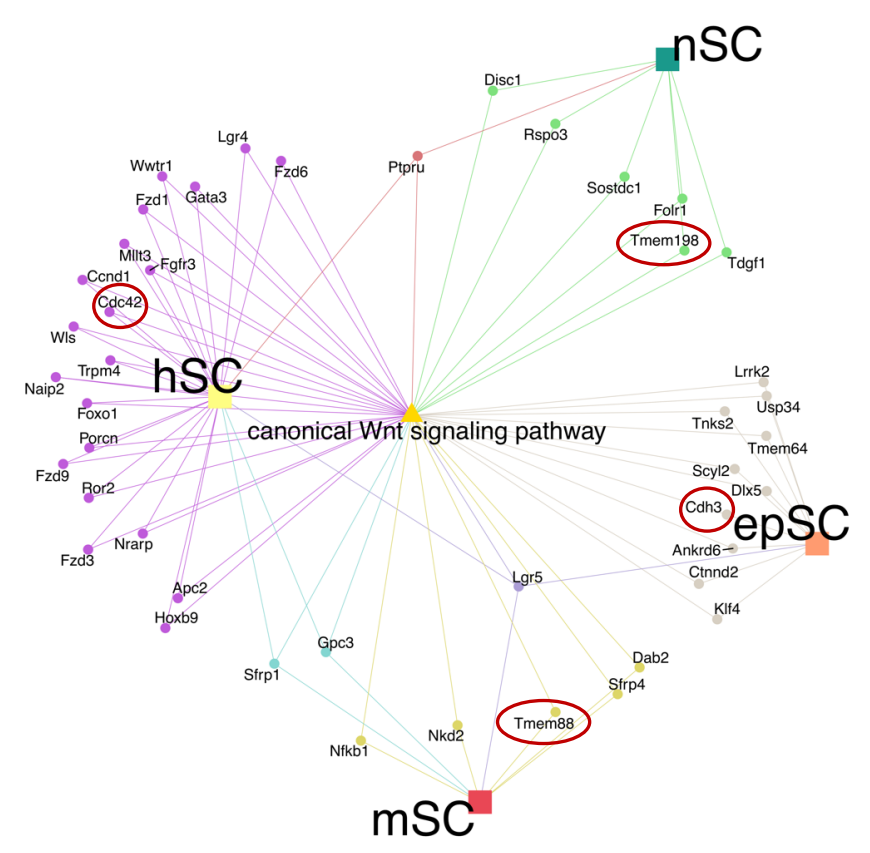


Figure S7. String gene network analysis for cell signaling pathways, related to Figure 4.

(A) String gene network analysis showing unique or shared upregulated genes that are responsible for cytokine-mediated signaling pathway in stem cell niches.

(B) String gene network analysis showing unique or shared upregulated genes that are responsible for regulation of TGF $\beta$ signaling pathway in stem cell niches.

(C) String gene network analysis showing unique or shared upregulated genes that are responsible for negative regulation of apoptotic signaling pathway in stem cell niches.

(D) String gene network analysis showing unique or shared upregulated genes that are responsible for cell surface receptor signaling pathway in stem cell niches.

(E) String gene network analysis showing unique or shared upregulated genes that are responsible for transmembrane receptor protein serine/threonine kinase signaling pathway in stem cell niches.

(F) String gene network analysis showing unique or shared upregulated genes that are responsible for canonical WNT signaling pathway in stem cell niches. 


\section{Figure $\mathbf{S 8}$}

A Nuclease-containing compound biosynthetic process

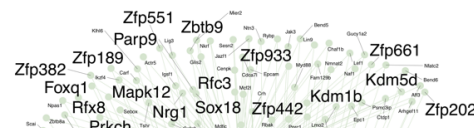
Zip641 Prkch

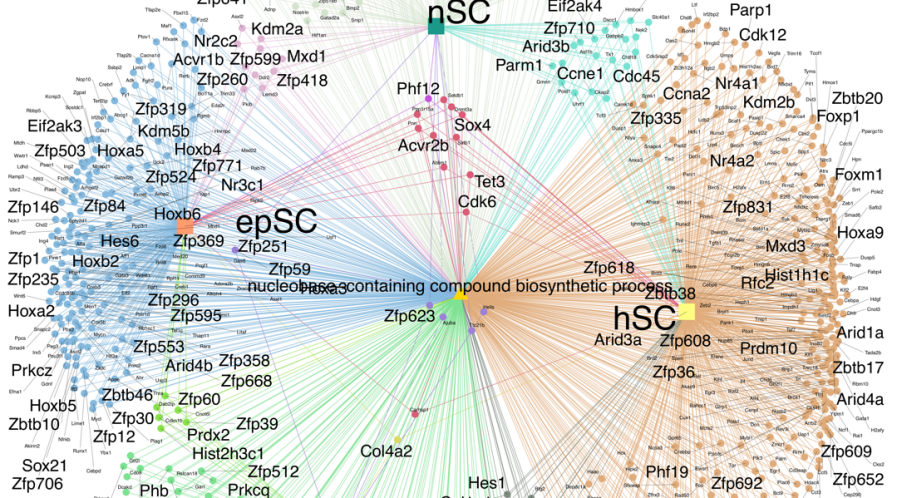

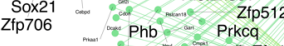

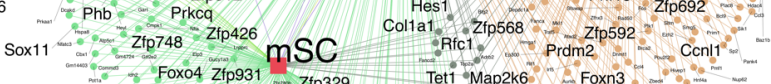

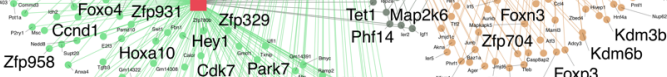

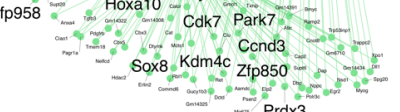

\section{Transcription, DNA templated}

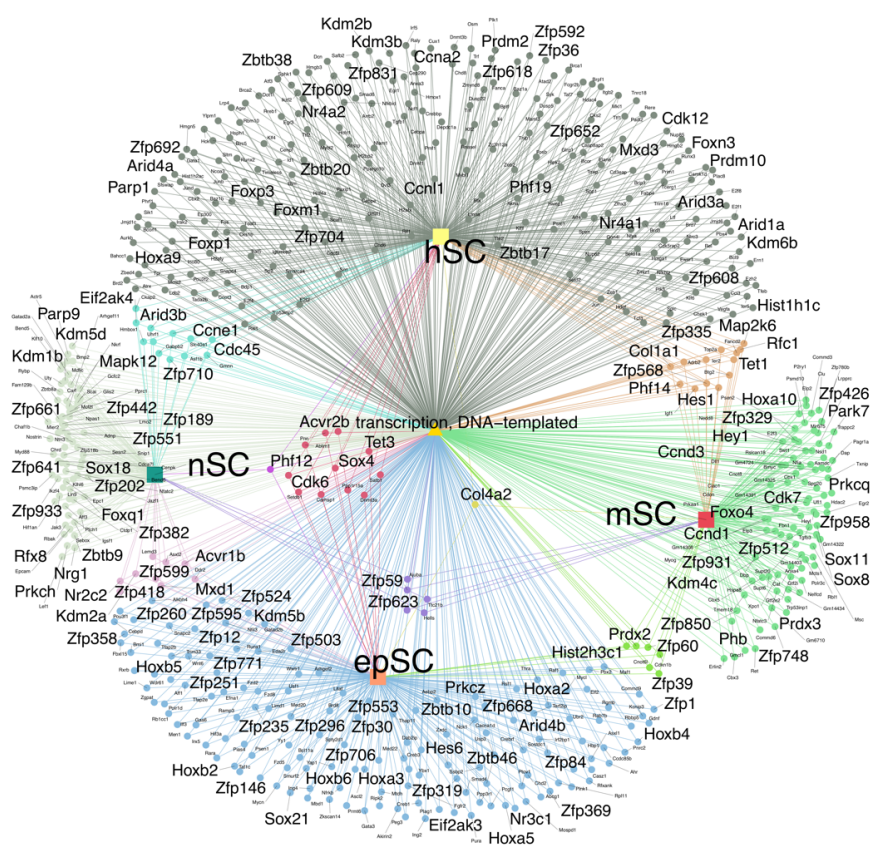

B

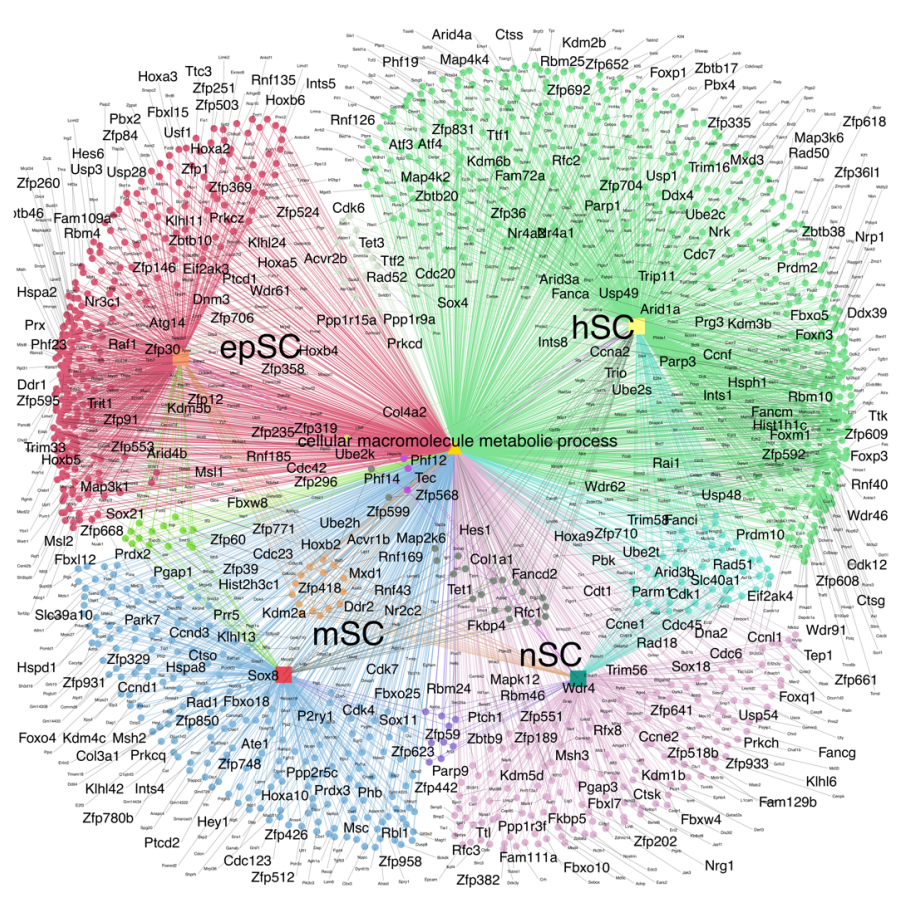

E

\section{RNA metabolic process}

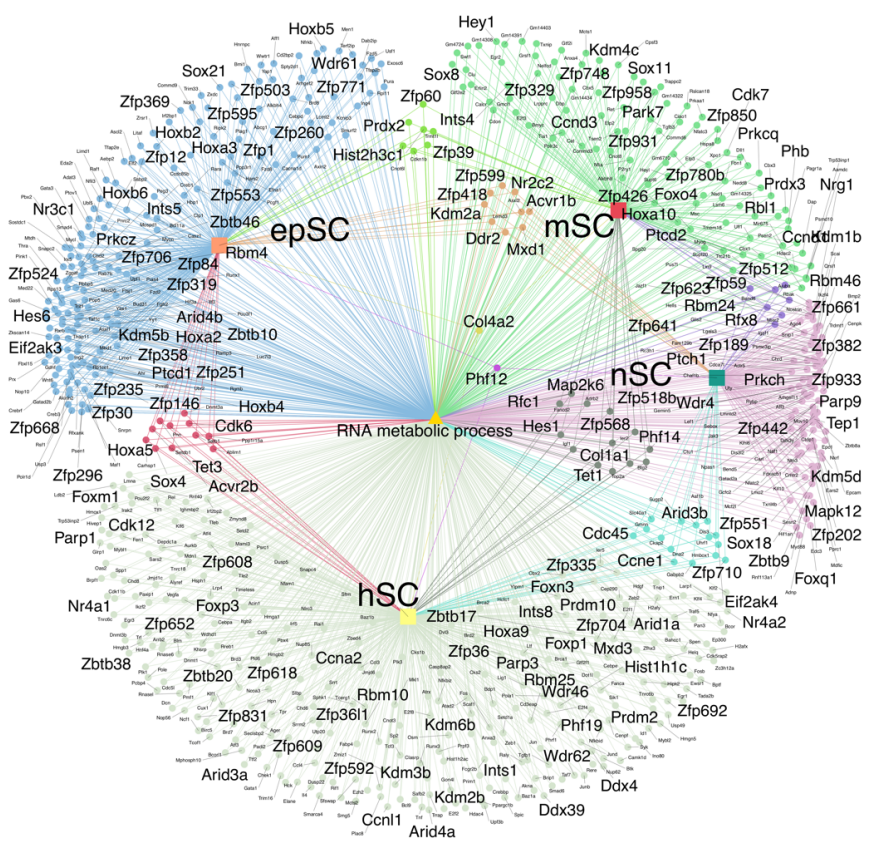

C Nucleic acid metabolic process

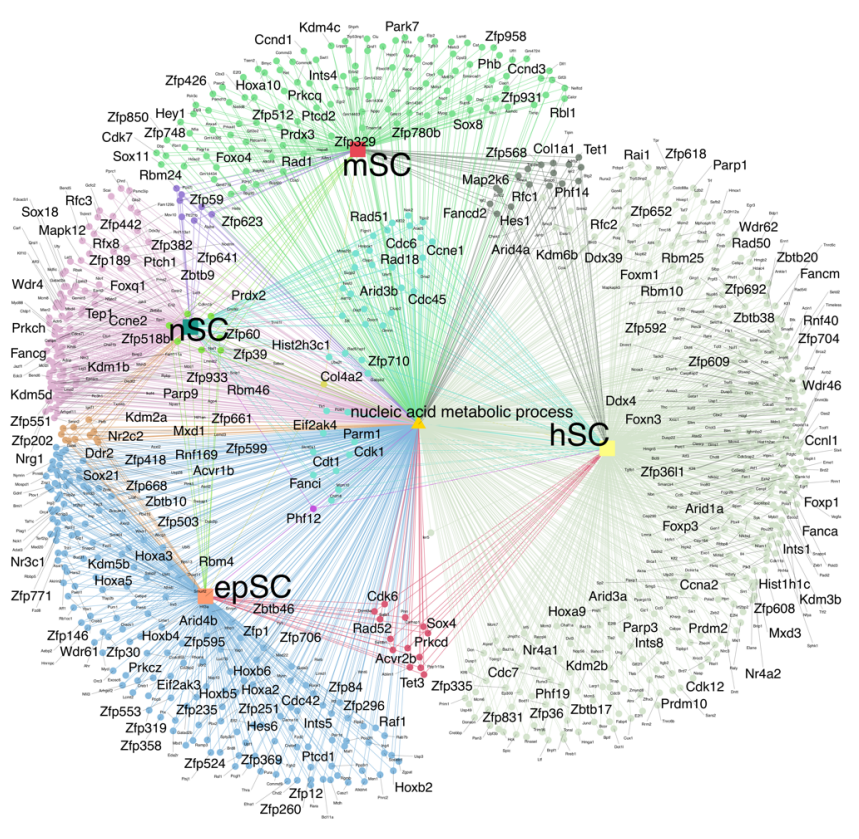

F RNA biosynthesis processes

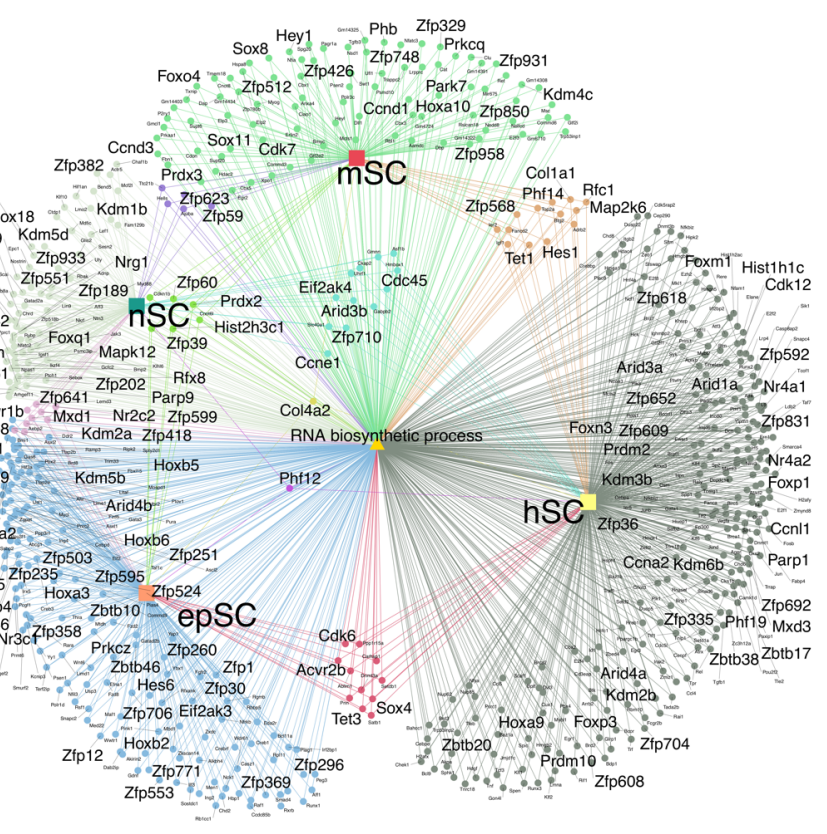


Figure S8. String gene network analysis for RNA homeostasis, related to Figure 4.

(A) String gene network analysis showing unique or shared down-regulated genes that are responsible for nuclease - containing compound biosynthetic process in stem cell niches.

(B) String gene network analysis showing unique or shared down-regulated genes that are responsible for cellular macromolecule metabolic process in stem cell niches.

(C) String gene network analysis showing unique or shared down-regulated genes that are responsible for nucleic acid metabolic process in stem cell niches.

(D) String gene network analysis showing unique or shared down-regulated genes that are responsible for transcription, DNA-templated in stem cell niches.

(E) String gene network analysis showing unique or shared down-regulated genes that are responsible for RNA metabolic process in stem cell niches.

(F) String gene network analysis showing unique or shared down-regulated genes that are responsible for RNA biosynthetic processes in stem cell niches. 


\section{Figure S9}

A

Cellular catabolic process

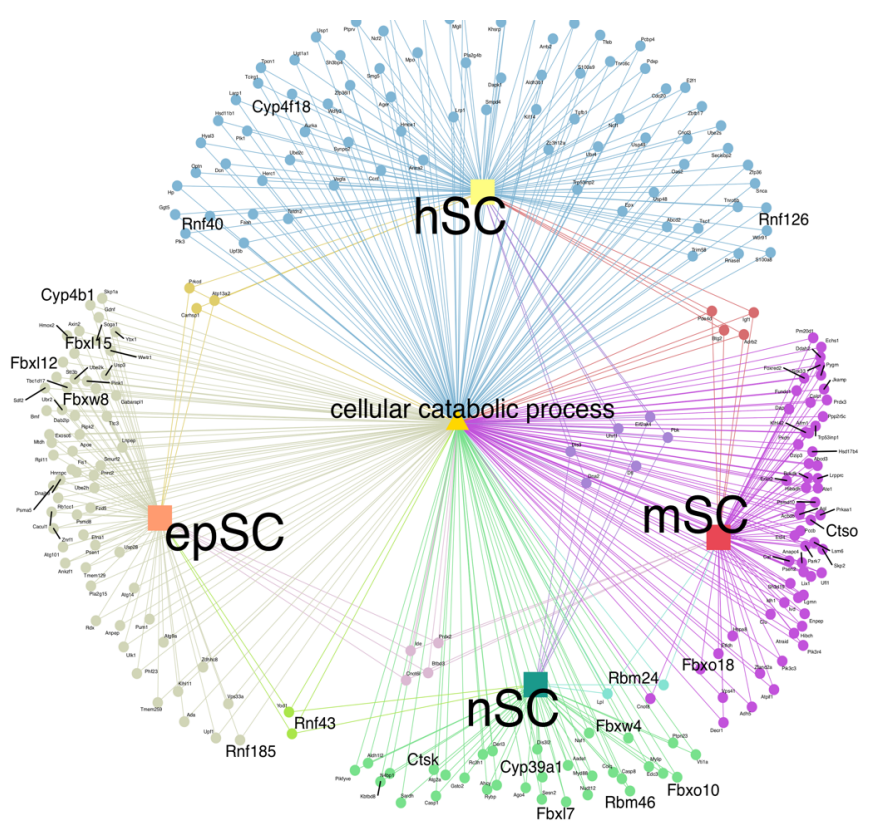

D Cellular protein metabolic process

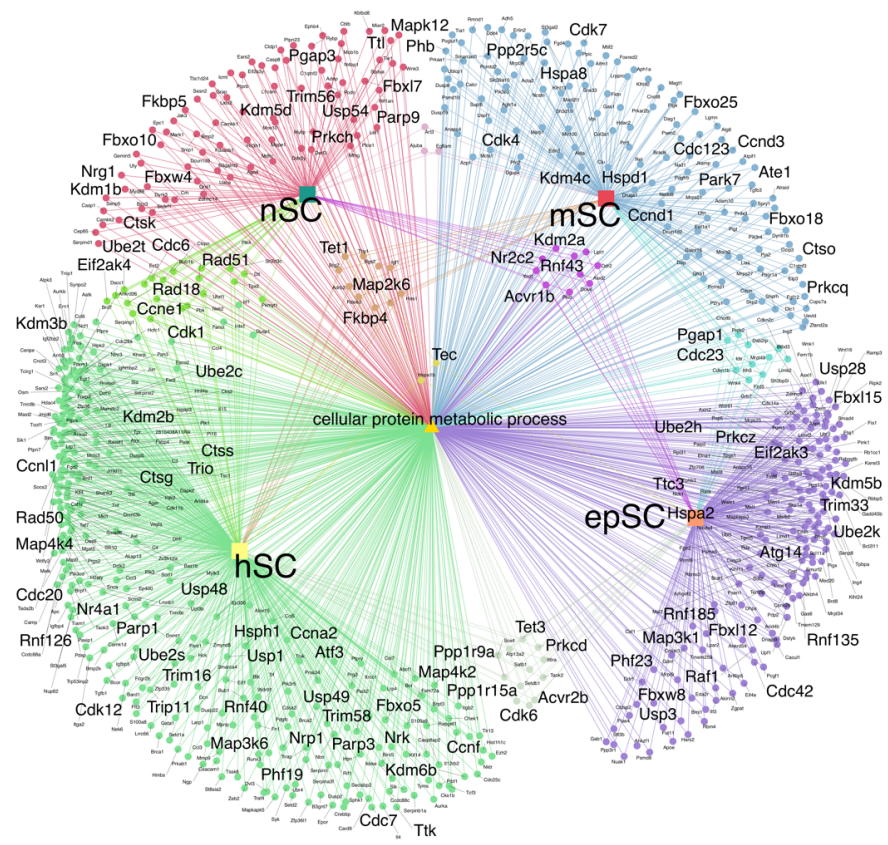

B Regulation of cellular protein metabolic process

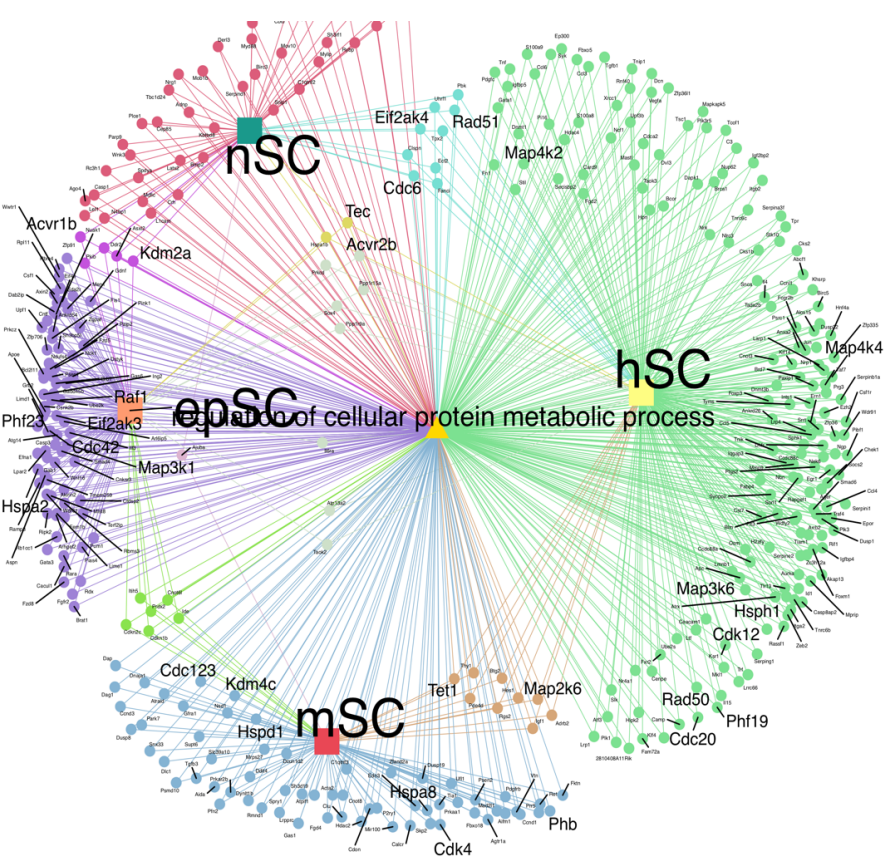

E

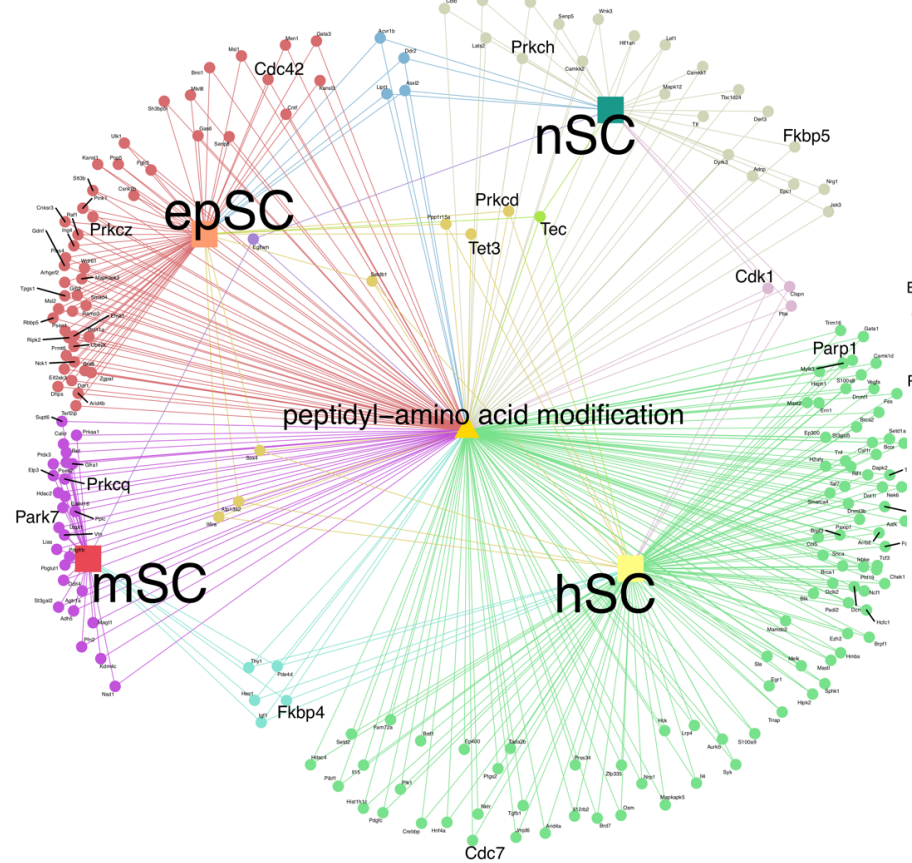

C Phosphate-containing compound metabolic process

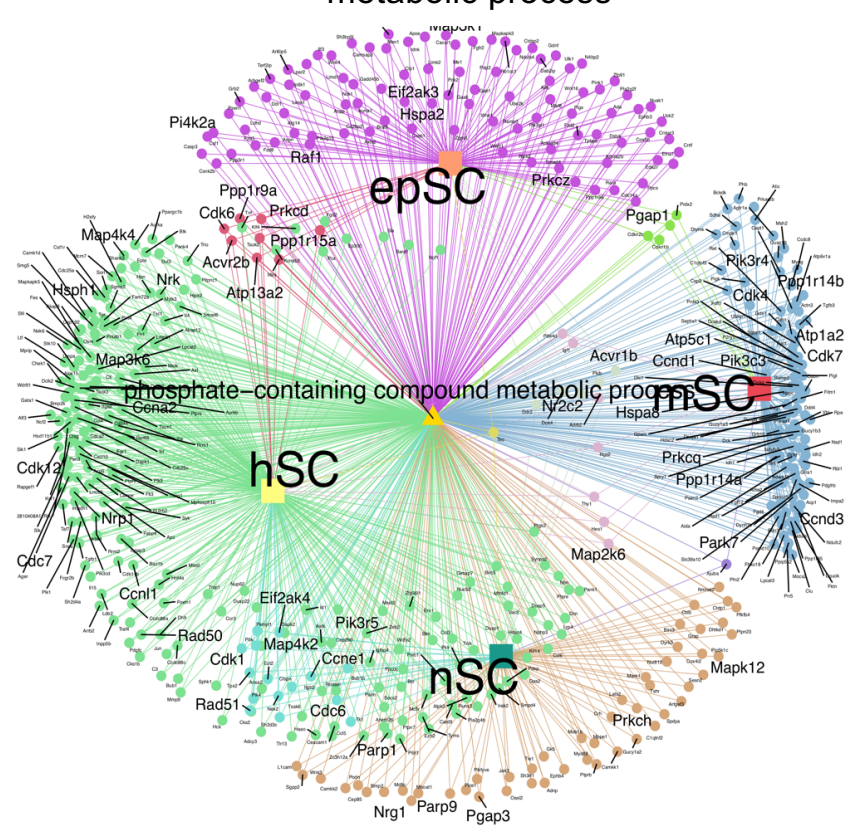

F Phosphorylation 
Figure S9. String gene network analysis for protein homeostasis, related to Figure 4.

(A) String gene network analysis showing unique or shared down-regulated genes that are responsible for cellular catabolic process in stem cell niches.

(B) String gene network analysis showing unique or shared down-regulated genes that are responsible for regulation of cellular protein metabolic process in stem cell niches.

(C) String gene network analysis showing unique or shared down-regulated genes that are responsible for phosphate - containing compound metabolic process in stem cell niches.

(D) String gene network analysis showing unique or shared down-regulated genes that are responsible for cellular protein metabolic process in stem cell niches.

(E) String gene network analysis showing unique or shared down-regulated genes that are responsible for peptidyl-amino acid modifications in stem cell niches.

(F) String gene network analysis showing unique or shared down-regulated genes that are responsible for phosphorylation in stem cell niches. 